\title{
IS IT POSSIBLE TO DISCOVER A DARK MATTER PARTICLE WITH AN ACCELERATOR?
}

\author{
VADIM A. BEDNYAKOV \\ DLNP, JINR, RUSSIA
}

\begin{abstract}
Aвstract. The paper contains description of the main properties of the galactic dark matter (DM) particles, available approaches for detection of DM, main features of direct DM detection, ways to estimate prospects for the DM detection, the first collider search for a DM candidate within an Effective Field Theory, complete review of ATLAS results of the DM candidate search with LHC RUN I, and less complete review of "exotic" dark particle searches with other accelerators and not only.

From these considerations it follows that one is unable to prove, especially modelindependently, a discovery of a DM particle with an accelerator, or collider. One can only obtain evidence on existence of a weakly interacting neutral particle, which could be, or could not be the DM candidate.

The current LHC DM search program uses only the missing transverse energy signature. Non-observation of any excess above Standard Model expectations forces the LHC experiments to enter into the same fighting for the best exclusion curve, in which (almost) all direct and indirect DM search experiments permanently take place. But this fighting has very little (almost nothing) to do with a real possibility of discovering a DM particle. The true DM particles possess an exclusive galactic signature - annual modulation of a signal, which is accessible today only for direct DM detection experiments. There is no way for it with a collider, or accelerator.

Therefore to prove the DM nature of a collider-discovered candidate one must find the candidate in a direct DM experiment and demonstrate the galactic signature for the candidate. Furthermore, being observed, the DM particle must be implemented into a modern theoretical framework. The best candidate is the supersymmetry, which looks today inevitable for coherent interpretation of all available DM data.
\end{abstract}

\section{Dark matter particles are Stable, neutral, with a Clear galaxy feature}

Galactic Dark Matter (DM) particles do not emit or reflect any detectable electromagnetic radiation and clearly manifest themselves today only gravitationally by affecting other astrophysical objects. Numerous observational indications at astronomical and cosmological scales [1, 2, 3, 4, 5, 6, 7, 8, 9, 10, 11, as well as results from very sophisticated numerical many-body simulations of genesis of cosmic large- and small-scale structures (see, for example, [12]), indicate the presence of this new form of matter in the Universe.

In particular, stars and gas clouds in galaxies and galaxies in clusters move faster than can be explained by the pull of visible matter alone. Light from distant objects may be distorted by the gravity of intervening dark material. The pattern of the large-scale structures across the 
Universe is largely dictated by DM. In fact, about $85 \%$ of the Universe's mass is dark, accounting for about a quarter of the total cosmic energy budget [2]. Some of recent reviews on the subject can be found also in [13, 14].

For further consideration the local density (nearby the solar system) and local distribution of the relic DM-particle population are both very important [15]. To allow a measurable direct detection event rate in a modern DM-detector it must be not very low [6, 7, 10]. According to estimates based on a detailed model of our Galaxy [16] the local density of DM amounts to about $\rho_{\text {local }}^{\text {DM }} \simeq 0.3 \mathrm{GeV} / \mathrm{cm}^{3} \simeq 5 \cdot 10^{-25} \mathrm{~g} / \mathrm{cm}^{3}$. Recent studies argue that the current best-fit value for the local DM density, which should be used as a benchmark for direct DM detection searches, is $0.4-0.5 \mathrm{GeV} / \mathrm{cm}^{3}$ [6, 17. The local flux of DM particles, which can cross the Earth, is expected to be $\Phi_{\text {local }}^{\mathrm{DM}} \simeq 10^{5} \frac{100 \mathrm{GeV}}{m_{\mathrm{DM}}} \mathrm{cm}^{-2} \mathrm{~s}^{-1}$, where $m_{\mathrm{DM}}$ is the DM particle mass. In other words, one can expect that $1 \mathrm{~cm}^{2}$ of the Earth's surface meets about $10^{5} \mathrm{DM}$ particles each second, provided their mass equals to $100 \mathrm{GeV} / c^{2}$. This value is often considered as a promising basis for laboratory direct DM search experiments.

Furthermore, the Big Bang conception of the early Universe [18] strongly supports the idea of non-gravitational coupling of the DM particles with the ordinary matter. This interaction could be very weak, but not completely vanishing.

Despite many other possibilities [19] the Weakly Interacting Massive Particle (WIMP) is among the most popular candidates for the relic DM. Being electrically neutral and interacting rather weakly, the WIMPs naturally reproduce the correct relic DM abundance, if their masses coincide with a typical new physics TeV-scale $-M_{\mathrm{WIMP}} \leq \frac{g^{2}}{0.3} 1.8 \mathrm{TeV}[20]$. This coincidence adds extra interest to the search for the DM particles, especially directly with the LHC, which is nowadays the best artificial $\mathrm{TeV}$-scale-physics explorer.

These particles are non-baryonic and there is no room for them in the Standard Model of particle physics (SM), in particular due to the Big Bang nucleosynthesis, which successfully predicts the abundances of light elements such as deuterium, helium and lithium arising from interactions in the early Universe. Furthermore, to explain the way in which galaxies form and cluster, these massive DM particles should be non-relativistic, or so-called "cold DM" particles. If they were relativistic, they could easily travel beyond the typical scale of a protogalaxy, and galaxy-scale structures would not have chance to appear [2].

Therefore one needs a New Physics beyond the SM (BSM). The lightest supersymmetric (SUSY) particle (LSP), the neutralino, in many R-parity conserving model realizations of SUSY (MSSM, NMSSM, mSUGRA, etc) - being massive, neutral, stable and possessing correct relic abundance - very naturally plays the role of the WIMP DM particle.

The primary goal of modern particle physics and astrophysics is to detect the DM particles that constitute the massive invisible halo of the Milky Way.

One believes [2] that in spite of decades of compelling efforts, all attempts to detect DM particles have failed so far (with one important exception of DAMA/LIBRA results). This "DM Problem" is a real challenge for modern physics and experimental technology. To solve the problem, i.e. at least to detect these DM particles, one simultaneously needs to apply the front-end knowledge of modern particle physics, astrophysics, cosmology and nuclear physics. 
Furthermore one should develop and have in long-term usage an extremely high-sensitive setup, to say nothing about complex data analysis methods (see, for example, discussion in [21]).

It is clear why so many papers concerning the DM problem are published. The papers discuss new DM candidates, new DM detection methods and new technologies for the DM search, new strategies and new models, etc. Nevertheless, on this way one point almost always stays in shadow, and this is the galactic origin and galactic belonging of the searched-for and to a much greater extent, ever registered DM candidates. The nature must be clearly used for the search strategy and especially to prove the registration of the "true" DM particles.

\section{How does one Want to Detect the DM?}

There is a common belief that the DM problem can be solved by means of a complete and balanced research program based on the following four categories.

Direct Detection experiments look for a direct DM interaction in an underground terrestrial low-background laboratory, where a DM particle scatters off a (nuclear) substance of a detector, producing a detectable recoil and/or ionization signal.

Indirect Detection experiments are unable to detect DM directly, but with (huge) terrestrial setups try to register products of DM particle annihilations in cosmic objects like the Earth, the Sun, our own and/or another galaxy. It is assumed that pairs of DM particles annihilate each other producing high-energy ordinary particles (antimatter, neutrinos, photons, etc). In some models, the DM particles can be metastable and eventually decay with production of SM particles.

Particle Collider experiments can help one to understand the properties of the DM particles. The LHC and future lepton and hadron colliders can produce energetic DM particles that obviously will escape detection, but could be registered by means of an excess of events with missing energy or momentum.

Astrophysical Probes provide one with information about non-gravitational interactions of DM particle populations, such as DM densities in the centers of galaxies and cooling regimes of stars. The particle properties of DM are constrained here through observation of their joint impact on astrophysical observables. Examples include self-interaction of DM particles affecting central DM densities in galaxies (inferred from the rotation velocity or velocity dispersion), mass of DM particle affecting the DM substructure in galaxies (inferred from strong lensing) and annihilation of DM in the early Universe affecting the Cosmic Microwave Background (CMB) fluctuations [22].

These search strategies are schematically shown in Fig. 1. Each of these approaches has its own advantages and disadvantages. Therefore, one speaks about different types of complementarities of them [13, 22].

Below some words are said about the indirect detection experiments and the astroparticle probes. The direct detection technique and searches with colliders are discussed in sections 3 and 5, 6 in more detail.

The idea of the indirect detection relies on a set of reasonable assumptions. One believes that a DM particle on its way through the space can be attracted by a rather massive cosmic body, like the Sun, and one day can be trapped by the gravitational potential of the body. Afterwards this particle oscillates in the potential and passes many-many times through the 


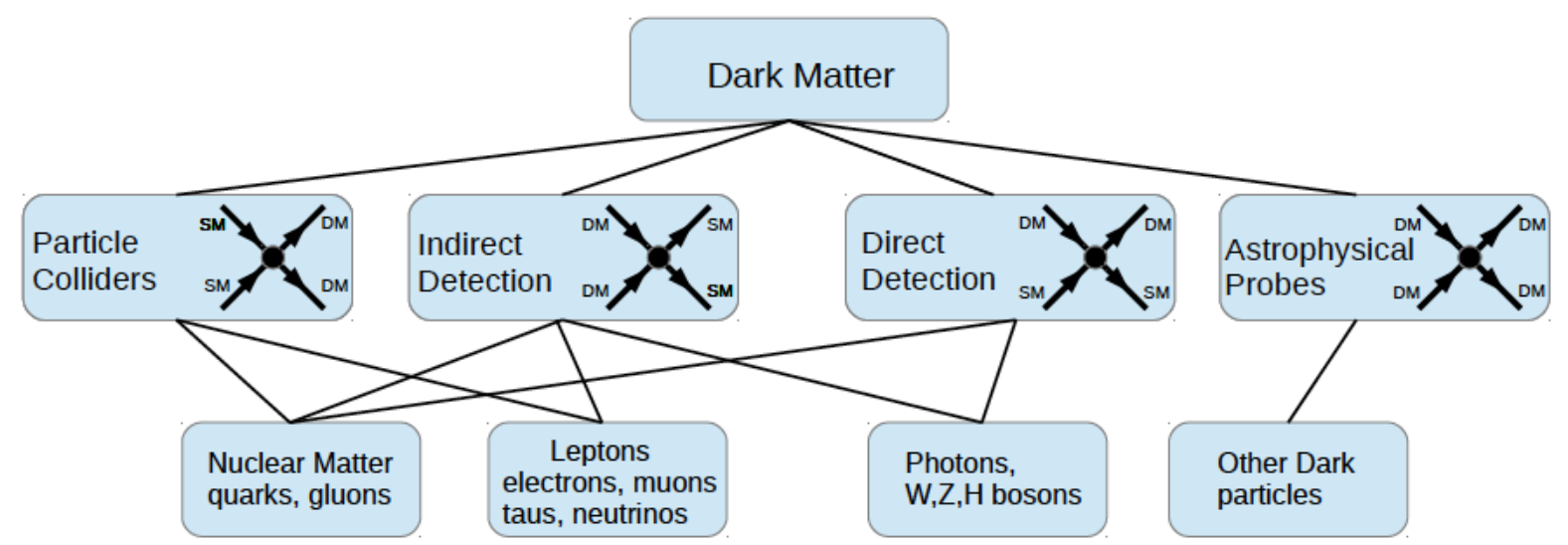

FIgURE 1. The idea of DM particle registration with a terrestrial setup relies on the common belief that DM can interact non-gravitationally with nuclear matter, leptons, gauge and other bosons, and with other possible dark particles. These interactions may be studied nowadays with direct and indirect detection techniques, particle colliders, and via astrophysical probes [22].

ordinary matter of the body. In the course of this inevitable travel the WIMP nature of the DM plays a crucial role, the particle eventually loses its kinetic energy due to a non-vanishing weak interaction and after some time sinks into the deepness of the body. After accumulation of a critical amount of the DM particles within the body they start a permanent process of mutual annihilation (again via weak-scale interaction). The annihilation products in a due course leave the body in the form of fluxes of $\gamma$-rays, X-rays, neutrinos, electrons and positrons, to a much less extent, antiprotons and antideuterons. Registration of these fluxes with a proper terrestrial setup constitutes the indirect detection of DM. Therefore the aim of the indirect DM search is registration of the processes in outer space that were responsible for modern relic DM density. These (mainly annihilation) processes are still under way, especially in some space regions where the local DM density can strongly exceed the average relic DM density [23].

The indirect detection technique includes [24, 25, 26] space and ground-based gamma-ray telescopes like the Fermi Large Area Telescope (Fermi-LAT) [27], cosmic ray detectors, large underground, under-ice and underwater Cherenkov neutrino telescopes like Super-Kamiokande (see, for example, [28]), IceCube (see, for example, [29]), ANTARES [30] and BAIKAL (see, for example, [31]).

The main disadvantage of the indirect detection technique is the "ordinary" astrophysics, which can easily produce irreducible backgrounds [23]. Indeed, one must conclude that until now all attempts to detect DM indirectly have been inconclusive [2].

In particular, an excess of positrons in the cosmic-ray spectrum up to $350 \mathrm{GeV}$, reported by the Alpha Magnetic Spectrometer (AMS-02) on the International Space Station (ISS) [32], can be due to the DM particle annihilations. The excess strengthened previous results from the Payload for Antimatter Matter Exploration and Light-nuclei Astrophysics (PAMELA) satellite [33. Nevertheless these "extra" positrons can be produced by other sources, such as winds from rapidly rotating neutron stars [2]. Further observations of positrons with AMS-02 at higher energy might distinguish between these hypotheses [34]. 
Recently, the AMS-02 collaboration has reported results of the cosmic-ray antiproton measurement. Antiprotons can be a product of annihilation or decay of galactic DM. It was claimed in [34, 35, 36] that this $\bar{p}$ flux does not exceed uncertainties of the astrophysical secondary $\bar{p}$ flux. Nevertheless the expected secondary antiproton flux has a tendency to be smaller than the observed one at energy $\geq 100 \mathrm{GeV}$, allowing one to still think about a DM contribution in that energy range [37, 38].

Another excess of $\gamma$-rays, in the form of a narrow line at $130 \mathrm{GeV}$, from the Galactic center where DM particles can concentrate and annihilate, was observed by the Fermi Gamma-ray Space Telescope [39, 40]. But a similar line from the Earth's atmospheric limb implies that at least part of the signal must be instrumental by origin [2, 41]. The High Energy Stereoscopic System (HESS) $\gamma$-ray telescope in Namibia (see, for example, [42]), which is observing the inner Galaxy in the $100 \mathrm{GeV}$ to $1 \mathrm{TeV}$ energy range, can resolve the situation in coming years. Preferred in these cases, DM particle masses are expected to be in the range $10-40 \mathrm{GeV} / \mathrm{c}^{2}$ and are rather sensitive to details of the electron interstellar propagation [2]. The existence of DM with these masses can also be probed by the temperature fluctuations of the CMB. The fluctuations would be damped by any delay in recombination, which is expected for such mass DM particles annihilation. The lower the mass, the more ionizing photons are produced for any cosmologically specified DM density. It is anticipated that the Planck satellite will soon set a more definitive constraint on self-annihilating DM particle masses below $30-40 \mathrm{GeV} / c^{2}$ [2].

There are also plans for a variety of sensitive gamma-ray telescopes in the energy range 1-100 $\mathrm{MeV}$ aimed at indirect study of low-mass DM annihilation or decay [43, 44, 45].

Simultaneously, one looks for more massive (TeV-scale) particles, which could be difficult to detect directly, because one should expect much smaller number density of them to fit current DM density. The gamma-ray astronomy has no such a limitation. The international Cherenkov Telescope Array (CTA) with more than 100 ground-based dedicated telescopes is planned to capture Cherenkov light flashes from $\gamma$-rays scattered by the atmosphere. The CTA will be able to measure $\gamma$-rays with $100-\mathrm{TeV}$ energy, which could be generated by annihilations or decays of DM with 100-TeV scale masses. This energy scale reaches the highest limit on the DM mass expected from fundamental physics arguments [25]. With GAMMA-400 [46] $\gamma$-ray telescope one expects new results in the energy range $0.1-3000 \mathrm{GeV}$ [47, 48, 49, 50].

An investigation of the angular cross-correlation of non-gravitational signals with low-redshift gravitational probes is proposed in [51] as a most powerful technique to detect DM signal outside the Local Group. This technique is more sensitive than other extragalactic $\gamma$-ray probes, such as the energy spectrum and angular autocorrelation of the extragalactic background, and emission from clusters of galaxies. In particular, the measured cross-correlation can be explained by a DM particle, with thermal annihilation cross section and mass between 10 and $100 \mathrm{GeV} / c^{2}$ [51].

More information about indirect DM search experiments can be found, for example, in [13, 52].

Due to unprecedented accuracy of astronomical observations there are today many different variants for the astrophysical probes (assays) of the DM existence in the sky.

For example, one can look for a large number of starless DM halos surrounding the Milky Way, which are not yet detected but well expected in modern cosmology and astrophysics as the cold DM. These DM halos, in the form of clumps or streams, can move through or orbit the Milky Way and can increase substantially the direct DM detection rates together with production of 
rather small, but detectable, velocity changes of the stars in the galaxy disk. These kinematic signatures will be detected by the new space telescope GAIA [53] provided the starless DM halos take place in the sky [54].

Next, the amount of DM accumulated in the neutron stars together with the energy deposition rate from the DM decays could set a limit on the neutron star survival rate against transitions to more compact objects provided that DM is indeed unstable. This limit sets constraints on the DM lifetime [55].

Furthermore, one should look for some unusual signals in old neutron stars and white dwarfs. In particular, the DM particles accumulated in the core of a neutron star in the course of its long-term travel through the Galaxy might form a tiny black hole that could eventually devour the home star, causing a very unusual explosion [56. The influence of DM particles collected in the Sun on the solar temperature variation could also be probed by helioseismology [2]. Merging galaxy clusters such as the Bullet Cluster can provide a powerful testing ground for galactic DM observation [57]. There are also proposals to consider a possibility of galactic DM interactions with cosmic rays and different kinds of interstellar matter [58]. In the case of multicomponent galactic DM sector with at least two DM species with different masses one can use the DM-to-DM decays as a new complementary tool for investigation of the DM properties [59].

The potential of microwave background lensing to probe the DM distribution in galaxy group and galaxy cluster halos was demonstrated in 60]. Evidence was presented of the gravitational lensing of the cosmic microwave background by $10^{13}$ solar mass DM halos. The mean lensing signal is consistent with simulated DM halo profiles.

From the cosmological point of view one can put the following few constraints on DM. DM must have the correct cosmological energy density, it must be massive so that it can act as pressureless matter. DM particles should not interact so strongly as to either disturb the wellunderstood $\mathrm{CMB}$ or to fail to collapse sufficiently to explain the observed large-scale structure of the Universe [23].

In order to convincingly establish the nature of a DM candidate, one must reach consistency between all possible DM searches for the common DM candidate parameters of mass, spin, and coupling strengths [20].

\section{Main features of the DiRect DM Detection}

The direct DM detection has a bit exceptional status among the other DM search techniques discussed above. The reasons could be a rather old history of this approach, existence of the DAMA evidence (see below), and a possibility of supporting us with the clearer and most decisive information on the DM problem [61].

One should absolutely agree with the authors of [2], that "... the goal is to detect the particles that constitute the massive halo of dark matter that surrounds the Milky Way, as they pass through our detectors ..." Another argument is [6] that "... until dark matter particles are detected in the laboratory, it is also healthy to remember that there are hints that the dark sector might be more complicated ..." Perhaps unconsciously, the decisive role of the laboratory direct DM detection experiments was stressed here.

Due to elastic WIMP-nucleus scattering the nuclear recoil energy is the main quantity to be measured by a terrestrial detector in direct DM detection laboratory experiments [62]. Detection 
of very rare events of such WIMP interactions is a quite complicated task because of very weak WIMP coupling with ordinary matter. The rates expected, for example, in SUSY models range from 10 to $10^{-7}$ events per kilogram detector material a day [63, 64, 65, 66, 67]. Moreover, for WIMP masses between a few $\mathrm{GeV} / c^{2}$ and $1 \mathrm{TeV} / c^{2}$ the energy deposited by the recoil nucleus is much less than $100 \mathrm{keV}$.

Therefore, in order to be able to detect a WIMP, an experiment should have an "ideal" detector with 1) a large target mass of an isotope (preferably with a high mass number $A$ ), 2) a low energy threshold (several keV), 3) an ultra-low radioactive background, 4) a possibility of distinguishing the signal (nuclear recoils) from the background (electronic recoils), and 5) in order to reduce the cosmic-ray induced background, this detector has to be installed in a deep-underground laboratory, where the overburden almost completely eliminates the hadronic component of the cosmic rays and reduces the muon flux by 5-7 orders of magnitude [68]. Additionally one should have a possibility of running the setup for several years.

All these complications, especially the non-zero energy threshold, the need for a large target mass and rather long exposure time, are sometimes considered as a kind of limitations in the sensitivity of the direct search experiments [23].

According to [68, 69] two-phase time projection chambers (TPCs) filled with liquid (and gaseous) xenon (LXe) are considered today as the most sensitive technique for direct WIMP search in an underground experiment. This statement relies on a (very) large homogeneous target of a very low background and a possibility of localizing the interaction vertex. The latter allows fiducial volume control and rejection of multiple-scatterring events. The background events in the setup are further rejected by the simultaneous measurement of the scintillation and ionization signals (Fig. 2).
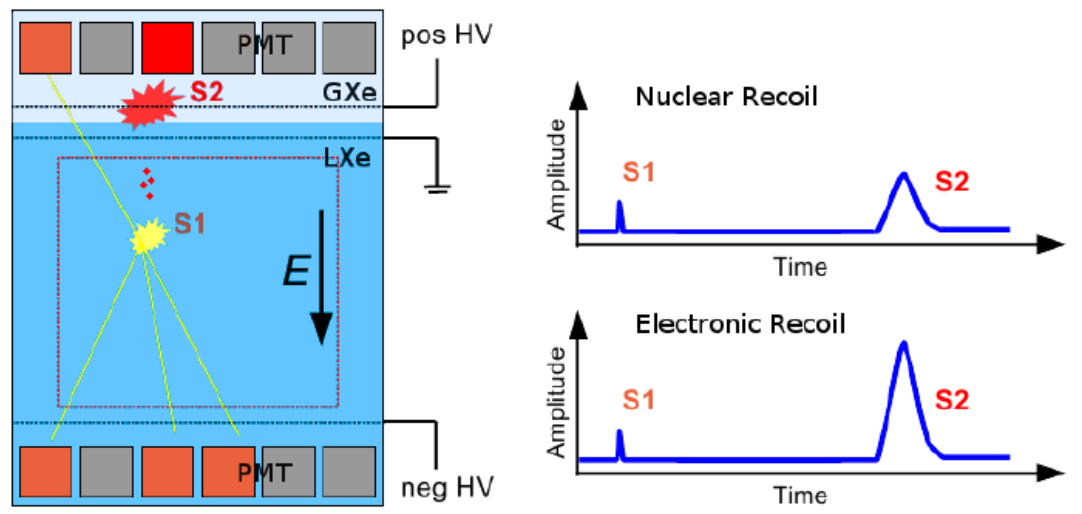

Figure 2. A dual phase time projection chamber measures scintillation light (S1) and the ionization charge signal, which is converted to a proportional scintillation signal (S2) in the xenon gas phase (GXe). The time distance between the two signals and the pattern on the top PMT array is used to reconstruct the event vertex. The ratio S2/S1 is different for nuclear and electronic recoils and used for background discrimination From [68.

Perhaps, one of the best examples of such a technique today is the XENON100 experiment, which constitutes (with $161 \mathrm{~kg}$ of ultra pure LXe) the second phase of the XENON program [70] for the direct DM detection. After 224.6 live days of running no indication for an excess 
of events above the expected background has been observed with the XENON100, leading to strong upper limits for relevant WIMP-nucleon scattering cross sections [71, 72].

In the nearest future the LUX experiment is expected to reach its design goals [73, 74]. The other dual-phase LXe experiment is the Chinese experiment PandaX [75, 76] with a flat pancake-shaped target of $120 \mathrm{~kg}$ LXe (25 kg fiducial mass). At the famous LNGS underground laboratory, the XENON collaboration constructs a new XENON1T setup [77, 78] with a target mass of $2.2 \mathrm{t}$, which will be the first TPC for the WIMP search with a mass of about $1 \mathrm{t}$. The goal of XENON1T is to perform a background-free DM search run with an exposure of 2 tonne $\times$ years. The expected sensitivity is at a level of $2 \times 10^{-47} \mathrm{~cm}^{2}$ at a WIMP mass of $50 \mathrm{GeV} / c^{2}$. One believes that all sub-systems of XENON1T can be re-used in a later upgrade phase of the experiment, XENONnT, with about $7 \mathrm{t}$ of LXe [68]. These projects will significantly improve the sensitivity to WIMP-nucleon interactions by 1-2 orders of magnitude compared to the present status [69].

There are many other running direct DM search experiments [79, 80, 73, 81, 82, 83, 84, 85, 71, 86, 87, 88, 89, 90, 91]. The results of these experiments are given in the figures of section 6 in connection with the collider DM search results of the ATLAS collaboration. The experimental status of the direct DM experiments is comprehensively discussed in [4, 92, 61, 68, 69, 13.

3.1. Current situation - fight for the best exclusion curve. The nuclear recoil energy $E_{\mathrm{R}}$ produced by a DM WIMP with mass $m_{\chi}$ is measured by a DM detector. The differential event rate (the spectrum) has the form

$$
S(t) \equiv \frac{d R}{d E_{\mathrm{R}}}=N_{A} \frac{\rho_{\mathrm{local}}^{\mathrm{DM}}}{m_{\chi}} \int_{v_{\min }}^{v_{\max }} d v f(v) v \frac{d \sigma^{A}}{d q^{2}}\left(v, q^{2}\right) .
$$

The nuclear recoil energy $E_{\mathrm{R}}=q^{2} /\left(2 M_{A}\right)$ is typically about $10^{-6} m_{\chi}, N_{A}$ is the number density of target nuclei with mass $M_{A}, v_{\max }=v_{\mathrm{esc}} \approx 600 \mathrm{~km} / \mathrm{s}$, and $v_{\min }=\left(M_{A} E_{\mathrm{R}} / 2 \mu_{A}^{2}\right)^{1 / 2}$ is the minimal WIMP velocity which still can produce the recoil energy $E_{\mathrm{R}}$. The WIMP-nucleus differential elastic scattering cross section for spin-non-zero $(J \neq 0)$ nuclei contains the coherent (spin-independent, or SI) and axial (spin-dependent, or SD) terms [93, 94]

$$
\frac{d \sigma^{A}}{d q^{2}}\left(v, q^{2}\right)=\frac{\sigma_{\mathrm{SD}}^{A}}{4 \mu_{A}^{2} v^{2}} F_{\mathrm{SD}}^{2}\left(q^{2}\right)+\frac{\sigma_{\mathrm{SI}}^{A}}{4 \mu_{A}^{2} v^{2}} F_{\mathrm{SI}}^{2}\left(q^{2}\right) .
$$

The normalized nuclear form-factors $F_{\mathrm{SD}, \mathrm{SI}}^{2}\left(q^{2}\right)$ are expressed in terms of the nuclear structure functions given in [93, 94]. For $q=0$ the nuclear SD and SI cross sections can be represented as

$$
\begin{aligned}
\sigma_{\mathrm{SI}}^{A} & =\frac{\mu_{A}^{2}}{\mu_{p}^{2}} A^{2} \sigma_{\mathrm{SI}}^{p}, \\
\sigma_{\mathrm{SD}}^{A} & =\frac{4 \mu_{A}^{2}}{\pi} \frac{(J+1)}{J}\left\{a_{p}\left\langle\mathbf{S}_{p}^{A}\right\rangle+a_{n}\left\langle\mathbf{S}_{n}^{A}\right\rangle\right\}^{2}=\frac{\mu_{A}^{2}}{\mu_{p}^{2}} \frac{4}{3} \frac{J+1}{J} \sigma_{\mathrm{SD}}\left\{\left\langle\mathbf{S}_{p}^{A}\right\rangle \cos \theta+\left\langle\mathbf{S}_{n}^{A}\right\rangle \sin \theta\right\}^{2} .
\end{aligned}
$$

The effective spin cross section $\sigma_{\mathrm{SD}}$ and the coupling mixing angle $\theta$ were introduced [95]:

$$
\sigma_{\mathrm{SD}}=\frac{\mu_{p}^{2}}{\pi} \frac{4}{3}\left[a_{p}^{2}+a_{n}^{2}\right], \quad \tan \theta=\frac{a_{n}}{a_{p}} ; \quad \sigma_{\mathrm{SD}}^{p}=\sigma_{\mathrm{SD}} \cdot \cos ^{2} \theta, \quad \sigma_{\mathrm{SD}}^{n}=\sigma_{\mathrm{SD}} \cdot \sin ^{2} \theta .
$$

Here, $\mu_{A}=\frac{m_{\chi} M_{A}}{m_{\chi}+M_{A}}$ is the reduced mass and $\mu_{n}^{2}=\mu_{p}^{2}$ is assumed. The dependence on the effective WIMP-quark scalar $\mathrm{C}_{q}$ and axial-vector $\mathrm{A}_{q}$ couplings $\left(\mathrm{A}_{q} \cdot \bar{\chi} \gamma_{\mu} \gamma_{5} \chi \cdot \bar{q} \gamma^{\mu} \gamma_{5} q+\mathrm{C}_{q} \cdot \bar{\chi} \chi \cdot \bar{q} q\right)$ 
and on the spin $\left(\Delta_{q}^{(p, n)}\right)$ and the scalar $\left(f_{q}^{(p)} \approx f_{q}^{(n)}\right)$ structure of the nucleons enter via the zero-momentum-transfer WIMP-proton and WIMP-neutron SI and SD cross sections

$$
\begin{aligned}
\sigma_{\mathrm{SI}}^{p}=4 \frac{\mu_{p}^{2}}{\pi} c_{0}^{2}, & c_{0}=c_{0}^{p, n}=\sum_{q} \mathrm{C}_{q} f_{q}^{(p, n)} \\
\sigma_{\mathrm{SD}}^{p, n}=12 \frac{\mu_{p, n}^{2}}{\pi} a_{p, n}^{2} & a_{p}=\sum_{q} \mathrm{~A}_{q} \Delta_{q}^{(p)}, \quad a_{n}=\sum_{q} \mathrm{~A}_{q} \Delta_{q}^{(n)} .
\end{aligned}
$$

The factors $\Delta_{q}^{(p, n)}$, which parameterize the quark spin content of the nucleon, are defined as $2 \Delta_{q}^{(n, p)} s^{\mu} \equiv\left\langle p, s\left|\bar{\psi}_{q} \gamma^{\mu} \gamma_{5} \psi_{q}\right| p, s\right\rangle_{(p, n)}$. The quantity $\left\langle\mathbf{S}_{p(n)}^{A}\right\rangle=\left\langle A\left|\sum_{i}^{A} \mathbf{s}_{p(n)}^{i}\right| A\right\rangle$ is the total spin of protons (neutrons) averaged over all $A$ nucleons of the nucleus $(A, Z)$. For the direct DM detection isotopes either $\left\langle\mathbf{S}_{p}^{A}\right\rangle$ or $\left\langle\mathbf{S}_{n}^{A}\right\rangle$ dominates: $\left\langle\mathbf{S}_{n(p)}^{A}\right\rangle \ll\left\langle\mathbf{S}_{p(n)}^{A}\right\rangle$ [96, 97, 98. The differential event rate (1) can be rewritten in the form [95, 99]

$$
\begin{aligned}
\frac{d R\left(E_{\mathrm{R}}\right)}{d E_{\mathrm{R}}} & =\kappa_{\mathrm{SI}}\left(E_{\mathrm{R}}, m_{\chi}\right) \sigma_{\mathrm{SI}}+\kappa_{\mathrm{SD}}\left(E_{\mathrm{R}}, m_{\chi}\right) \sigma_{\mathrm{SD}} \\
\kappa_{\mathrm{SI}}\left(E_{\mathrm{R}}, m_{\chi}\right) & =N_{T} \frac{\rho_{\chi} M_{A}}{2 m_{\chi} \mu_{p}^{2}} B_{\mathrm{SI}}\left(E_{\mathrm{R}}\right)\left[M_{A}^{2}\right], \quad B_{\mathrm{SI}, \mathrm{SD}}\left(E_{\mathrm{R}}\right)=\frac{\langle v\rangle}{\left\langle v^{2}\right\rangle} F_{\mathrm{SI}, \mathrm{SD}}^{2}\left(E_{\mathrm{R}}\right) I\left(E_{\mathrm{R}}\right), \\
\kappa_{\mathrm{SD}}\left(E_{\mathrm{R}}, m_{\chi}\right) & =N_{T} \frac{\rho_{\chi} M_{A}}{2 m_{\chi} \mu_{p}^{2}} B_{\mathrm{SD}}\left(E_{\mathrm{R}}\right)\left[\frac{4}{3} \frac{J+1}{J}\left(\left\langle\mathbf{S}_{p}\right\rangle \cos \theta+\left\langle\mathbf{S}_{n}\right\rangle \sin \theta\right)^{2}\right] .
\end{aligned}
$$

The dimensionless integral $I\left(E_{\mathrm{R}}\right)$ accumulates properties of the DM velocity distribution

$$
I\left(E_{\mathrm{R}}\right)=\frac{\left\langle v^{2}\right\rangle}{\langle v\rangle} \int_{x_{\min }} \frac{f(x)}{v} d x=\frac{\sqrt{\pi}}{2} \frac{3+2 \eta^{2}}{\sqrt{\pi}\left(1+2 \eta^{2}\right) \operatorname{erf}(\eta)+2 \eta e^{-\eta^{2}}}\left[\operatorname{erf}\left(x_{\min }+\eta\right)-\operatorname{erf}\left(x_{\min }-\eta\right)\right] .
$$

Here one assumes the Maxwell-Boltzmann DM velocity distribution in the rest frame of the Galaxy, $\eta$ denotes the dimensionless Earth's speed with respect to the halo, and $x_{\min }^{2}=\frac{3}{4} \frac{M_{A} E_{\mathrm{R}}}{\mu_{A}^{2} \bar{v}^{2}}$ [100, 64]. The error function is $\operatorname{erf}(x)=\frac{2}{\sqrt{\pi}} \int_{0}^{x} d t e^{-t^{2}}$. The velocity variable is the dispersion $\bar{v} \simeq 270 \mathrm{~km} / \mathrm{c}$. The mean velocity $\langle v\rangle=\sqrt{\frac{5}{3}} \bar{v}$. Integrating the differential rate 11 from the recoil energy threshold $\epsilon$ to some maximal energy $\varepsilon$, one obtains the total detection rate $R(\epsilon, \varepsilon)$ as a sum of the SD and SI terms

$$
R(\epsilon, \varepsilon)=R_{\mathrm{SI}}(\epsilon, \varepsilon)+R_{\mathrm{SD}}(\epsilon, \varepsilon)=\int_{\epsilon}^{\varepsilon} d E_{\mathrm{R}} \kappa_{\mathrm{SI}}\left(E_{\mathrm{R}}, m_{\chi}\right) \sigma_{\mathrm{SI}}+\int_{\epsilon}^{\varepsilon} d E_{\mathrm{R}} \kappa_{\mathrm{SD}}\left(E_{\mathrm{R}}, m_{\chi}\right) \sigma_{\mathrm{SD}}
$$

To accurately estimate the event rate $R(\epsilon, \varepsilon)$, one needs to know a number of quite uncertain astrophysical and nuclear structure parameters as well as very specific characteristics of the experimental setup [21] (see also [101]).

Finally, to estimate the expected direct DM detection rates - via formulas (1) or (5) - one should calculate the WIMP-proton and WIMP-neutron spin $\sigma_{\mathrm{SD}}^{p, n}$ and scalar $\sigma_{\mathrm{SI}}^{p, n}$ cross sections at $q=0$. To this end a SUSY-like model or some measured data (for example, from the DAMA/LIBRA experiment) can be used. It is these calculations that are usually compared with experimental results, which are presented in the form of exclusion curves - upper limits of the cross sections as functions of the WIMP mass.

Figures 3 and 4 from [61] illustrate the typical situation in the field of direct DM search experiments. They contain a lot of exclusion curves that demonstrate the recent achievements 


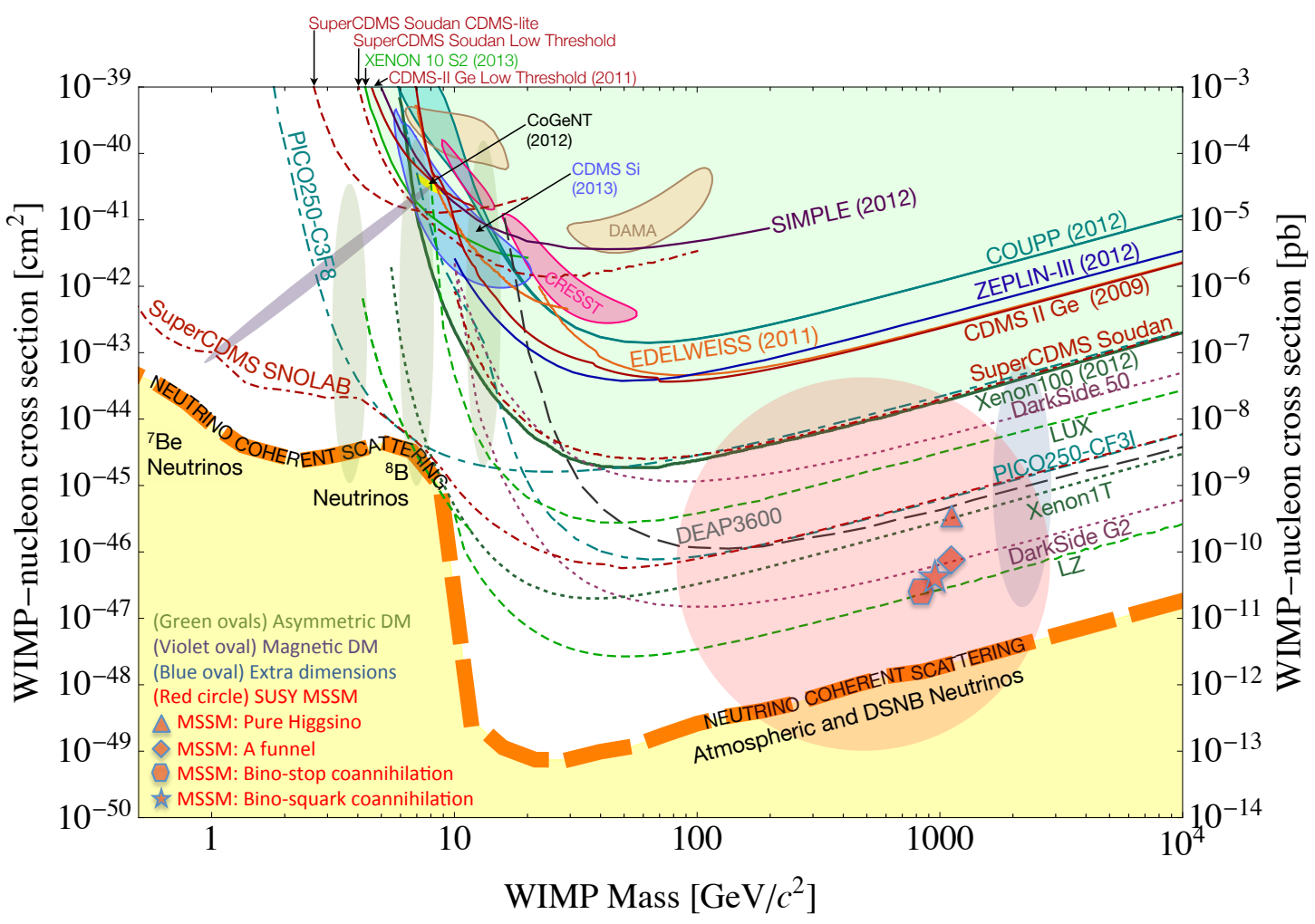

Figure 3. A compilation of WIMP-nucleon spin-independent cross section limits (solid curves), hints for WIMP signals (shaded closed contours) and projections (dot and dotdashed curves) for direct detection experiments that are expected to operate over the next decade. Also shown is an approximate band where coherent scattering of ${ }^{8} \mathrm{~B}$ solar neutrinos, atmospheric neutrinos and diffuse supernova neutrinos with nuclei will begin to limit the sensitivity of direct detection experiments to WIMPs. Finally, a suite of theoretical model predictions is indicated by the shaded regions, with model references included. From 61].

and expected improvements in sensitivity for both $\sigma_{\mathrm{SI}}$ and $\sigma_{\mathrm{SD}}$ WIMP-nucleon cross sections together with a range of theoretical benchmarks [61].

In the case of non-observation of a DM signal the exclusion curve simply reflects the sensitivity of a given direct DM search experiment and potentially allows one to constrain some version of the SUSY-like model, provided the curve is sensitive enough. Therefore the best exclusion curve is currently the only aim of almost all direct DM search experiments (DAMA/LIBRA is a clear exception). The main competition between these experiments takes place right in the field of the exclusion curves.

It is important to note that without proper knowledge of the nuclear and nucleon structure it is not possible to extract reliable and useful information from direct DM search experiments (at least in the form of the $\sigma_{\mathrm{SD}}^{n, p}$ and $\sigma_{\mathrm{SI}}$ cross sections). However, astrophysical uncertainties, 

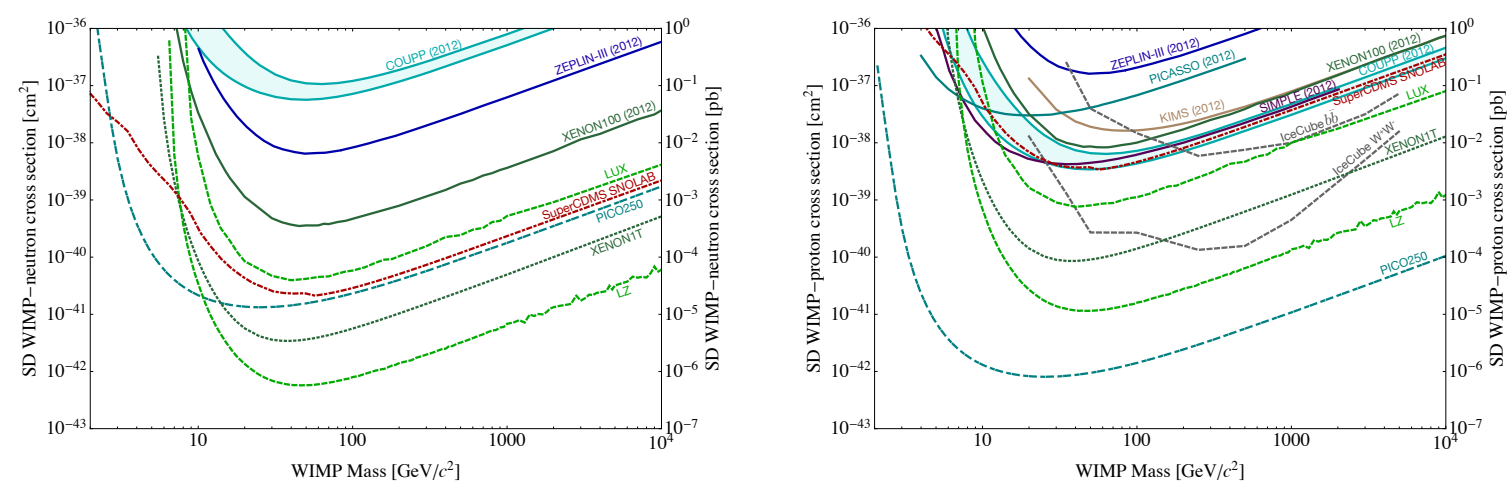

Figure 4. A compilation of WIMP-nucleon spin-dependent cross section limits (solid curves) and projections (dot and dot-dashed curves) for direct detection experiments that are expected to operate over the next decade. From 61.

in particular the DM distribution in the vicinity of the Earth, make it far more difficult to interpret the results of the DM search experiments. To have a chance to compare sensitivities of different experiments, people adopted a common truncated Maxwellian DM particle distribution but nobody can prove its correctness. In the case of undoubted direct DM detection one can make some conclusions about the real DM particle distribution in the vicinity of the Earth.

Furthermore, almost by definition (from the very beginning), a modern experiment aiming at the best exclusion curve is doomed to non-observation of the DM signal. This is due to the fact that a typical expected DM signal spectrum exponentially drops with recoil energy and it is practically impossible to single it out from the background non-WIMP spectrum of a typical detector. One needs a clear signature of interactions between WIMP particles and target nuclei. Ideally, this signature should be a unique feature of such an interaction [102]. Without these signatures one can hardly convince anybody that the final measured spectrum is saturated by the DM particles. Furthermore, on the basis of measured recoil spectra, with the help of these signatures, one can estimate the WIMP mass.

Therefore, exclusion curves hardly help to prove an observation of a DM signal. Nevertheless an exclusion curve is at least something from nothing observed. It allows a sensitivity comparison of different experiments and therefore one can decide who is the best "excluder". Unfortunately, the SUSY model paradigm is very flexible, it has a lot of parameters, and one hardly believes that an exclusion curve can ever impose any decisive constraint on it. Over many years the experimental groups compare obtained exclusion curves with SUSY (MSSM, NMSSM, etc) predictions. These comparisons permanently show that some extra domains of relevant parameter space are excluded. However, this long-term "exclusion business" has no practical benefit either for SUSY model construction, or planning of a new DM search. The situation is much worse due to the famous nuclear and astrophysical uncertainties involved in the evaluation of the exclusion curves. This is why it does not look very decisive to use refined data and methods and spend big resources fighting only for the best exclusion curve (a new information on the subject can be found in [103]). This fighting could only be accepted when one tries to strongly improve the 
sensitivity of a small DM detector with a view to use many copies of it in a huge detector array with a total tonne-scale mass [104].

In fact, all modern very sensitive and very low-background direct DM detectors are aimed at the best exclusion curve from the very beginning. They are looking for some event excess above a very low background, provided it is well understood. If one is the best in seeing nothing, a new best exclusion curve appears.

Furthermore, if one manages to find some events pretending to be the wanted excess, the powerful statistical likelihood technique allows one to quantify existence of this excess (in terms of $1-3 \sigma$ ) but does not allow one to prove the real detection of the DM interactions. Indeed, if a DM particle with mass $m_{\mathrm{DM}}$ exists and can interact with ordinary matter with some not-verysmall cross section $\sigma_{\mathrm{DM}}$, then an excess induced by these DM particles should be seen (better in a measured recoil spectrum). But the opposite is not correct. Any (or very specifically selected) excess (in the recoil spectrum) is not yet a proof of the existence of the DM-particle interaction with the detector material. It is not enough. One needs an unambiguous signature of the galactic nature of the observed interactions. The annual modulation component in the excess events could prove the DM nature of the excess.

3.2. Positive signatures and recoil spectra. The problem of DM particle detection is also very complicated due to a lack of any reliable indication where one should look for a signal of the DM interactions - the DM masses and DM-SM couplings are (in general) unknown. Therefore, to certainly detect a DM particle one has to unambiguously register some "positive signature" of the DM interaction with a target material. This positive signature must be very specific (ideally unique) only for the true DM interaction, and reliable experimental observation of the signature will serve as unambiguous proof of the DM detection.

There are some typical characteristics of WIMP DM particle interactions with a nuclear target which can potentially play the role of these positive DM signatures [104]. First of all, WIMPs produce nuclear recoils, whereas most radioactive backgrounds produce electron recoils. Nevertheless, for example, neutrons (and any other heavy neutral particle) can also produce nuclear recoils.

Due to the extremely rare event rate of the WIMP-nucleus interactions (the mean free path of a WIMP in matter is of the order of a light year), one can expect two features. One is the negligible probability of two consecutive interactions in a single detector or two closely located detectors. Multiple interactions of photons, $\gamma$-rays or neutrons under the same conditions are much more common. Therefore only non-multiple interaction events can be from the DM WIMPs. The other feature is a uniform distribution of the WIMP-induced events throughout a detector. This feature can be used to identify background events (from photons, neutrons, beta and alpha particles) in rather large-volume position-sensitive detectors.

The shape of the DM WIMP-induced recoil energy spectrum can be predicted rather accurately (for given WIMP mass, fixed nuclear structure functions, and astrophysical parameters). The observed energy-recoil spectrum, claiming to be from DM particles, must be consistent with the expectation. However, this shape is exponential, right as it is for many background sources. Unfortunately, the nuclear-recoil feature, the non-multiple interaction, the uniform event distribution throughout a detector, and the shape of the recoil energy spectrum could not be the clear "positive signature" of the DM interactions. 
The currently most promising, technically feasible and already used (by the DAMA collaboration) "positive signature" is the annual modulation signature [100, 64, 105]. The DM flux and its average kinetic energy vary annually due to the combined motion of the Earth and the Sun relative to the Galactic center. The impact DM energy increases (decreases) when the Earth velocity is added to (subtracted from) the velocity of the Sun (see Fig. 5). The recoil spectrum

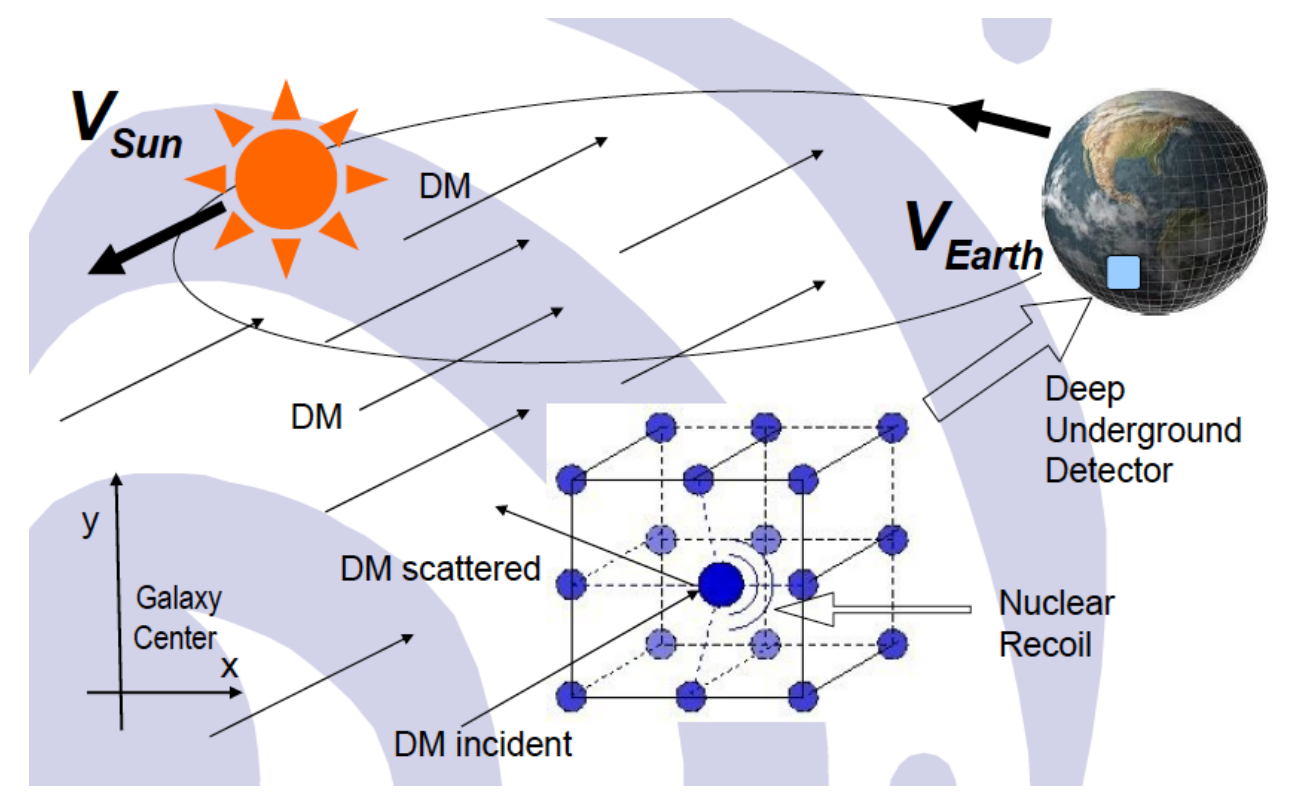

FiguRE 5. Detection of DM particles via elastic scattering from target nuclei in a detector located deep underground. Due to the expected annual modulation signature of the recoil measured event rate the Sun-Earth system is a particularly proper setup for successful direct DM detection [104].

produced from the DM-nucleus scattering in a target detector is therefore expected to show this annual modulation effect [100, 105].

The velocity of the Earth relative to the Galaxy is $v_{E}(t)=v_{S}+v_{O} \cos \gamma \cos \omega\left(t-t_{0}\right)$, where $v_{S}$ is the Sun's velocity relative to the Galaxy, $v_{O}$ is the Earth's orbital velocity around the Sun and $\gamma$ is the angle of inclination of the plane of the Earth's orbit relative to the galactic plane. One has $\omega=2 \pi / T$ ( $T=1$ year) and the maximum velocity occurs at day $t_{0}=155.2$ (June $2)$. The change in the Earth's velocity relative to the incident DM particles leads to a yearly modulation of the scattering event rate (1) in the form

$$
S(t)=S_{0}+S_{m} \cos \omega\left(t-t_{0}\right),
$$

where $S_{0}$ is the constant part and $S_{m}$ is the amplitude of the modulated signal. Both of them depend on the target nucleus $(A, Z)$, DM particle mass and density $\rho_{\text {local }}^{\text {DM }}$, velocity distribution of the DM particles in the solar vicinity $f(v)$, and cross sections of the DM-nucleus scattering (see, for example, [63, 64, 106, 66] and a new recent paper [107]).

In general, the expected modulation amplitude is rather small (about 7\%) [95, 108, 21] and to observe it, one needs huge (at best tonne-scale) detectors which can continuously operate during 5-7 years (to have a chance to observe the annual modulation effect). Of course, to reliably use this signature one should prove the absence of annually modulated backgrounds. This question is crucially investigated by the DAMA/LIBRA collaboration [108, 21]. 
Unfortunately, mainly due to not-yet-enough target mass and short running time, the abovementioned promising LXe based experiments seems to be unable to see a positive annual modulation signature of the DM interactions. Furthermore, to the best of our knowledge, they not ever aimed at fulfilling the goal.

Another potentially promising positive DM signature is connected with a possibility of registering the direction of the recoil nuclei induced by a DM particle. One plans to measure the correlation of the event rate with the Sun's motion. Unfortunately, the task is extremely complicated (see for example, [109, 110, 111]).

The third potentially useful positive WIMP DM signature is related with the coherence of the WIMP-nucleus spin-independent interaction. Due to a rather low momentum transfer, a WIMP coherently scatters by the whole target nucleus and the elastic cross section of this interaction should be proportional to $A^{2}$, where $A$ is the atomic number of the target nucleus. Contrary to the $A^{2}$ behavior, the cross section of neutron scattering by nuclei (due to the strong nature of this interaction) is proportional to the geometrical cross-section of the target nucleus $\left(A^{2 / 3}\right.$ dependence). To reliably use this $A^{2}$ signature, one has to satisfy at least two conditions. First, one should be sure that the spin-independent WIMP-nucleus interaction really dominates over the relevant spin-dependent interaction. This is far from being obvious [112, 99, 113]. Second, one should rather accurately measure the recoil spectra (in the worst case integrated event rates) under the same background conditions, at least for two targets with a different atomic number A. Currently, this goal looks far from being achievable.

At the level of our knowledge the DM problem could not be solved independently from the set of other related problems, such as proof of SUSY, astrophysical dark matter properties, etc. Furthermore, due to the huge complexity of the DM search (technical, physical, astrophysical, necessity for positive signatures, etc), one should, perhaps, deal with the DM problem boldly using a reliable model-dependent framework - for example the framework of SUSY, where the same LSP neutralino should be seen coherently (or lead to coherent effects) in all available experimental observables (direct and indirect DM searches, rare decays, high-energy searches at LHC, astrophysics, etc). A success of the SUSY framework will be a proof of the SUSY existence and simultaneous solution of the DM problem. In some sense, this SUSY framework can serve as a specific and very decisive and positive DM signature.

Ideally, in order to be convincing, an eventual DM signal should combine more than one of these positive DM signatures [102].

A physical reason (if one forgets about the above-mentioned competition of experiments) to improve the exclusion curve is usually an attempt to constrain a SUSY-like model. Unfortunately, this is almost hopeless due to the huge flexibility of these models and uncertainties from the nuclear structure and astrophysics. At the present level of experimental accuracy, simple fighting for the best exclusion curve is almost useless either for real DM detection or for substantial restriction of the models. One should go beyond the exclusion curve and try to obtain a reliable recoil energy spectrum. Very accurate off-line investigation of the measured spectrum allows one to single out different non-WIMP background sources and to perform controllable background subtractions. The spectrum allows one to look for the annual modulation effect, the only currently available positive signature of DM particle interactions with terrestrial nuclei. The effect is not simply a possibility of background rejection (among many others as claimed, 
for example, in [4]), it is a unique signature which demonstrates the galactic nature of the DM interaction with matter. This is inevitable for the laboratory proof of the DM population around the Earth [114.

Finally, the future requirements for 100-t scale direct DM detector, will meet a severe restriction - further sensitivity is strongly limited by an irreducible neutrino background (Fig. 3), mainly from supernovae, the Sun and neutrinos from cosmic rays [2, 115]. In this situation it is clear that to have progress in the DM detection a positive DM signature - the annual modulation - it is inevitable to fight against the irreducible $\nu$-background.

3.3. The DAMA/LIBRA evidence. Till now only the DAMA (DArk MAtter) collaboration has certainly given the evidence (at $9.3 \sigma \mathrm{CL}$ ) for the presence of DM particles in the halo of our Galaxy. The DAMA/LIBRA setups have in use a highly radio-pure $\mathrm{NaI}(\mathrm{Tl})$ target during 14 annual cycles (corresponding to cumulative exposure of 1.33 tonnexyears) at the deep underground Gran Sasso National Laboratory [95, 108, 21, 116]. The evidence is based on model-independent registration of changes in the flux of DM particles hitting the DAMA setup - the predicted annual modulation of specific shape and amplitude due to the combined motions of the Earth and the Sun through the Galaxy [100].

The DAMA/LIBRA measured modulation amplitude of the single-hit events is $(0.0112 \pm$ $0.0012)$ counts $/ \mathrm{day} / \mathrm{kg} / \mathrm{keV}$. The measured phase is $(144 \pm 7)$ days and the measured period is $(0.998 \pm 0.002)$ yr (Fig. 6). These values are well in agreement with those expected for true DM particles. This intriguing evidence of the DM detection is still very debated, simply because of its severe conflict with numerous null-results of almost all other direct DM search experiments [68. People widely discussed many other possible annual phenomena, such as, for example, neutrons leached from the rocks surrounding the underground experiment in response to seasonal temperature variations. Nevertheless, after many years of dedicated investigations of the DAMA collaboration no systematics or side reactions able to mimic the signature (that is, able to account for the measured modulation amplitude and simultaneously satisfy all the requirements of the signature) has been found or suggested [117, 116].

For completeness one should point out that indications on the similar annual cycles, consistent in phase with DAMA observations, were also seen (with much low statistical significance) by the CoGeNT (Coherent Germanium Neutrino Technology) [118, 119], CRESST-II [79], and CDMS-Si [81] DM-search experiments [69].

Despite the strong and reliable belief of the DAMA collaboration in the observation of the annual modulation signature, it is obvious that such a serious claim should be verified by at least another one completely independent experiment. If one wants to confirm (more important, if one wants to reject) the DAMA result, one should perform a new experiment which would have the same or better sensitivity to the annual modulation signature. Furthermore, it would be reasonable to locate a new setup in another low-background underground laboratory, and perhaps, in the Southern Hemisphere, where this new DM-modulation experiment would gauge the extent of the Earth's seasonal effects, which would be out of phase relative to ones in the Northern Hemisphere [2].

Finally, one can believe or cannot believe in the DAMA/LIBRA DM evidence, but to have a final scientific decision on the subject, one should prove experimentally the non-existence of the annual modulation effect observed by the DAMA/LIBRA collaboration at the $9 \sigma$ level. To this 

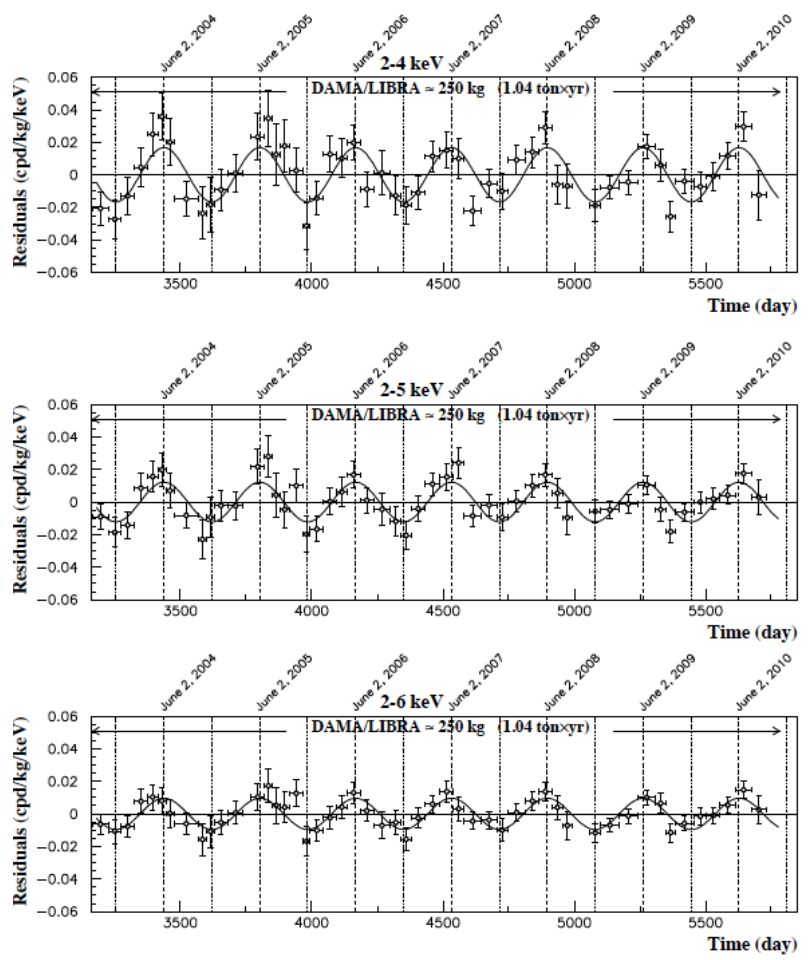

FiguRE 6. Experimental residual rate of the single-hit scintillation events measured by DAMA/LIBRA-phase1 in the (2-4), (2-5) and (2-6) keV energy intervals as a function of the time. The data points present the experimental errors as vertical bars and the associated time bin width as horizontal bars. The superimposed curves are $A \cos \omega\left(t-t_{0}\right)$ with a period $T=2 \pi / \omega=1 \mathrm{yr}$, a phase $t_{0}=152.5$ day (June 2nd) and modulation amplitudes, $A$, equal to the central values obtained by best fit on the data points of the entire DAMA/LIBRA-phase1. The dashed vertical lines correspond to the maximum expected for the DM signal (June 2nd), while the dotted vertical lines correspond to the minimum. From [116.

end one should first be able to ever see the effect in principle, to prove the proper sensitivity of a used or proposed setup.

\section{How Can one estimate the prospects for the DM Detection?}

Before designing a future DM detector one reasonably wants to have a feeling concerning how many events one could register during meaningful time of measurements with this detector (the expected event rate, section 3). A source of this information can be one of many recently appeared DM-models (section 7), or available experimental and astrophysical data. In the latter case one again should have a model that could simultaneously describe all the data and could give predictions for the DM rate via a unique set of parameters (as a SUSY-model can do).

Rather thorough investigations of prospects for direct DM detection have been carried out on the border of millennia on the basis of different versions of SUSY extension of the SM (MSSM, mSUGRA, NMSSM, etc). The main reason was the LSP - stable, neutral, massive, weaklyinteracting particle - an excellent (by-product) DM candidate in SUSY models with R-parity conservation. There are several main possibilities for the LSP in the MSSM - neutralino, 
gravitino, sneutrino, etc. The neutralino LSP was a particularly well-studied case (see, for example, [120]).

The modern SUSY framework is very appropriate for the goal. It allows one to describe simultaneously (with one set of SUSY parameters) all available experimental observables from very low up to very high energies. Therefore expected direct DM detection rates can be rather easily connected with probabilities of rare processes, results of high-energy measurements (LEP, FNAL), cosmological observations and restrictions on relic DM abundance (density).

For example, one can investigate the MSSM parameter space taking into account available accelerator, non-accelerator and cosmological constraints on the SUSY particle spectrum (upper bounds on their masses). On this basis one can obtain a reliable prediction for the expected SUSY DM detection rate in any high-accuracy DM detector (such as one with LXe). Nonobservation of the relevant DM candidates allows one to exclude some domains of the MSSM parameter space and make, for instance, predictions for charged Higgs boson search with a collider [121]. Furthermore, in this approach the MSSM Higgs bosons produced in the LSPneutralino self-annihilation in the Earth and the Sun could severely contribute to the total indirect DM detection rate. This contribution results in some lower bound for the muon flux from the Sun and one can expect an energetic $\tau$-neutrino flux from the Sun at a level of $10^{2}$ $\mathrm{m}^{-2} \mathrm{yr}^{-1}$ due to these charged Higgs bosons [122].

Nevertheless, at that time the high-energy colliders and accelerators were not yet considered (perhaps, due to relatively low energy, etc) as useful tools for "direct" collider DM search.

The unique LHC energy and the SUSY paradigm have opened new possibilities for connection of LHC experiments with the other DM searches. One can use available results from one kind of these experiments for goal-pointing for another one by means of some important hints on where one should search for a signal.

An example can be found in [123] where prospects for observation of a SUSY-like signal from two gluinos, were investigated within a certain region of the mSUGRA parameter space. In the region the lightest neutralinos $\chi_{1}^{0}$ are DM particles with $m_{\chi_{1}} \simeq 60 \mathrm{GeV} / c^{2}$ and naturally explain the excess of diffuse galactic $\gamma$-rays observed by the EGRET space apparatus. Additionally the cross-section of the two gluino production in pp-collisions at the LHC (14 TeV) was estimated at a level of $5-10 \mathrm{pb}$. Rather high transverse missing energy carried away by the two $\chi_{1}^{0}$ is the essential signature of these events that allows significant background reduction. Furthermore, distributions of the invariant masses of two opposite charged lepton pairs produced by the $\chi_{2}^{0} \rightarrow \chi_{1}^{0} l^{+} l^{-}$three-body decays have kinematic endpoints which measure the difference between the masses of $\chi_{2}^{0}$ and $\chi_{1}^{0}$. These signatures still demonstrate good prospects for discovery of the gluinos at the LHC.

The same SUSY-based interplay between the direct DM search and the LHC search for DM was considered earlier, for example in [124] and in [125]. In the latter paper, under an assumption that R-Parity conserving SUSY is already discovered at the LHC, a question of the LSP to be a real DM particle was addressed. In particular, the consistency of the observed SUSY realization (mSUGRA as an example) with astrophysical and non-accelerator constraints was studied and requirements for statistical and systematic accuracy of LHC measurements were formulated quantitatively. A discussion was given in [126] how one can constrain the underlying 
SUSY model and hence extract information about the nature of DM particles on the basis of techniques used to reconstruct decays of SUSY particles assumed to be observed at the LHC.

Recently a question of DM complementarity was studied in the 19-parameter phenomenological Minimal Supersymmetric Standard Model (pMSSM) including all available experimental constraints [127]. The ability to investigate the neutralino DM properties by the direct DM experiments XENON1T and LUX-ZEPLIN, by indirect DM searches with Fermi-LAT, CTA and IceCube, as well as by the LHC studies were examined. In particular, it was shown that expected LHC constraints on the pMSSM models are directly sensitive to many other sparticles beyond the LSP. Other sensitive tests of the pMSSM models are spin-independent direct detection with LUX-ZEPLIN and indirect detection through CTA. It was found that all discussed DM search techniques work well together within the MSSM and on this basis form a comprehensive program to determine DM properties [128].

Another investigation of the prospects of indirect and direct DM searches within the MSSM approach with 9 parameters (MSSM-9) was reported in [129]. A Monte Carlo scan of the parameter space was performed with allowance for all available particle physics constraints including the Higgs mass of $126 \mathrm{GeV} / c^{2}$. Two regions for DM were found with a TeV mass LSP neutralino. Prospects for future indirect (with the CTA) and direct (with XENON-1T) searches of these LSP were discussed and some search strategies were proposed [129]. Phenomenological study of the CMSSM (mSUGRA) with non-thermal neutralino DM candidate was carried out in [130].

Contrary to the MSSM in a GUT-defined SUSY model their parameters are no longer independent. The LHC prospects to probe a broad class of GUT-inspired SUSY models were studied in [131] with all available experimental constraints including the bounds from the muon $g-2$ anomaly, the DM relic density and the Higgs mass measurement.

Some version of the pMSSM was also used in [132] for explanation of an excess in $\gamma$-rays from the center of our Galaxy due to DM annihilation. The LSP neutralinos with mass about $90 \mathrm{GeV} / c^{2}$ provide a reasonable description of the excess, have correct relic density and are consistent with the other astroparticle and collider experiments. If the pMSSM explanation of the excess seen by Fermi-LAT is correct, a DM signal might be discovered soon - claimed the authors of [132].

Prospects of the detection of a GeV-scale neutralino as DM in the Next-to-Minimal Supersymmetric Standard Model (NMSSM) at the $14 \mathrm{TeV}$ LHC were studied in [133] by means of dedicated scans of the relevant parameter space including all available constraints. It was demonstrated in [134] that with a neutralino as DM candidate, the gamma-ray excess observed in the Fermi-LAT data cannot be accommodated in the MSSM. To reach the goal one needs the NMSSM with an extra singlet superfield and specific collider phenomenology.

There are some other investigations of DM detection prospects within SUSY-like models. For example, one believes [135] that in some SUSY models a right-handed sneutrino can be LSP and can play the role of a good DM candidate [136, 137, 138. Being a scalar particle this LSP will have the LHC signatures quite distinct from those one expects for the LSP-neutralino. Constraints on the sneutrino-LSP scenario were studied on the basis of general results of SUSY searches at RUN I of the LHC and most promising sneutrino signatures were proposed for further searches at RUN II. Phenomenological constraints on a light GeV-scale sneutrino as a 
DM particle were also investigated in [139]. How to find a "natural" supersymmetry via the interplay between the LHC and direct DM detection was discussed in [140].

Therefore, the "indirect" constraints on DM properties from collider physics were studied and used during many years. One of recent examples can be found also in [141], where constraints on DM from the first CMS and ATLAS supersymmetry searches are investigated. It was shown that within the minimal supergravity model (mSUGRA), the early search for SUSY (superpartners) at the LHC excluded a remarkable portion of the parameter space available for DM direct detection experiments. In particular, the prospects for detecting the SUSY DM (being the neutralino LSP) in the XENON and CDMS experiments are significantly affected in the low LSP mass region. In the case of non-universal soft breaking the regions excluded by minimal SUGRA, are not excluded at all. The authors allow an optimistic conclusion that an observation of DM (by direct detection) in the LHC excluded regions of mSUGRA might indicate non-universalities in soft breaking SUSY. Another important example of modern LHC influence on prospects of DM direct and indirect detection within SUSY can be found in a recent paper of Pran Nath [142]. This is a canonical way of collider data usage for direct/indirect DM search studies.

These few examples show that a properly developed model, especially the one like supersymmetric MSSM, mSUGRA, or NMSSM, can accurately unify information from all available energy scales and experiments and produce constraints or predictions for each of the DM search approaches listed in section 2. Therefore it is very reasonable to have in mind the SUSY-like background under the complementarily (or interplay) of all above-mentioned DM search opportunities.

\section{First "DireCT" DM SEarch With Tevatron}

Due to exciting results of the Tevatron and big expectations with the LHC, about 5 yeas ago a "direct" approach to the DM problem at colliders was proposed [143] and rapidly accepted by the collider community [144, 145, 146, 147, 148]. The main goal was to relate the pair production rate of DM candidates at colliders to the annihilation and scattering rates at direct and indirect DM experiments. It was believed that on this way (see, for example, [143, 145]) as few assumptions as possible should be made about unknown underlying new physics.

A true DM particle (section 1) is electrically neutral, massive enough, weakly interacting (WIMP), and stable. Therefore, the WIMP feature of the DM particle defines the very strategy of "direct" DM search with a collider. WIMPs, once produced, like neutrinos do not interact either electromagnetically or strongly with ordinary matter and pass numerous detectors layers without a trace. Their signature is invisibility. However the experiments ATLAS and CMS were designed to see this invisibility by means of an accurate measurement of energy deposited in the detectors by all other "visible" products of a hard LHC $p p$-collision. Hence, exploiting this hermeticity, one can judge the WIMP presence from the disbalance of the measured energy/momentum. Since the longitudinal momenta of the colliding partons are unknown, only the transverse missing energy, $E_{\mathrm{T}}^{\text {miss }}$, can be reliably used to search for the WIMP traces. In other words, hunting for WIMPs one should look for events with remarkable momentum imbalance, which is transverse to the initial proton beam line [149].

The collider searches for DM have their own advantages and disadvantages [150, 23]. Obviously, a collider search for WIMP does not suffer from astrophysical uncertainties. The search 
does not care about the existence of the Milky Way at all. Due to the famous low-recoil problem (small energy deposition) sensitivity of direct and indirect detection techniques almost vanishes with reduction of the WIMP mass. In contrast, colliders are able to copiously produce the light WIMPs (if production cross section is not very small), and one has no problem with sensitivity up to very low-mass WIMPs (if one finds a way to suppress neutrino background in this case). However, collider searches suffer from parton distribution function suppression for high WIMP masses (above hundreds of $\mathrm{GeV}$ ), where the other two search techniques are more robust.

Already at this stage a very important precaution [150, 23] says that a great disadvantage of any collider DM search is absence of any possibility of proving whether the observed $E_{\mathrm{T}}^{\text {miss }}$ signal is actually caused by the true DM particle or produced by a particle that is stable only on collider timescales, but not cosmological ones. The other techniques deal with the true DM particles produced long ago. Unfortunately this precaution almost always is forgotten or ignored (see discussion in section 8).

It looks like that the first quantitative discussions of a "direct" collider search for DM were based on the first mono-jet ( + large $E_{\mathrm{T}}^{\text {miss }}$ ) results of the CDF at the Tevatron and were given in [144] and in [143, 145]. These papers are rather remarkable. First, they have started to use the Effective Field Theory (EFT) approach in the subject, and next, from the very beginning many very serious assumptions have been used without any (or reasonable) proof.

Everybody will agree that true DM particles might be created at a collider. The very idea that direct DM detection requires an interaction of DM particles with ordinary matter (nucleons, nuclei, etc) is correct. But the point following intuitively from Fig. 1, that the same interaction can lead to the DM particle pair production at a hadron collider [144] far to be obvious. It needs to be well justified. Furthermore, in general it is wrong within such a complete theoretical model as SUSY. For example, in a R-conserved SUSY model the DM WIMPs are LSPs and usually constitute final products of cascade decays of heavier unstable SUSY-particles accompanied by SM particles, in particular, with high transverse momentum $p_{\mathrm{T}}$. Nevertheless as soon as one assumes that the same couplings lead to direct DM particle production at hadronic colliders such as the Tevatron, one can investigate the interplay between the two DM searches (within the EFT). One should also bear in mind that although the basic processes that work in direct detection and in collider production of DM occur through $s$ - and $t$-channel exchange of the same mediator, the regimes probed in the two types of the experiments are very different [144].

Due to the assumed DM nature (electric neutrality and stability), these WIMPs will leave any detector tracelessly, otherwise they could be detected (by ionization, interaction, or decay) and do not have a chance to serve as DM. Therefore signature of such escaping particles is (large) missing energy and momentum. More strictly, the latter is missing vector transverse momentum, $p_{\mathrm{T}}^{\text {miss }}$, the magnitude of which is called $E_{\mathrm{T}}^{\text {miss }}$ [151]. Unfortunately, only large transverse missing energy $E_{\mathrm{T}}^{\text {miss }}$ could be measured. To catch the signature one should search for visible particles recoiling against the WIMPs and triggering a relevant DAQ system.

This signature can be used to set constraints on the WIMP couplings to the constituents of nuclei, which in turn can be translated to constraints on direct detection cross sections [145].

The CDF collaboration has performed a search for one-jet events with large missing transverse energy $(\bar{p} p \rightarrow j+\chi \chi+X)$ using $1 \mathrm{fb}^{-1}$ of data [152]. . Here $\chi$ denotes the WIMP. These monojet searches at the Tevatron within the above-mentioned assumptions can be connected (via 
the same effective operators) to the DM direct detection searches and can place limits on the expected rates of the latter.

The analysis performed in [144] showed for the first time that in many cases the Tevatron provided the best limits, particularly for light WIMPs with mass below $5 \mathrm{GeV} / c^{2}$, and for SD WIMP-nucleon interactions. The bounds on the strength of the various effective operators were translated into bounds on direct detection rates. This enables one to plot the Tevatron limits - exclusion curves - in the $\sigma_{\mathrm{DM}}^{\mathrm{SI}, \mathrm{SD}}-m_{\mathrm{DM}}$ planes (as in Figs. 3 and 4 from section 3 ). The relevant figures can be found in [144].

In general these results confirmed the main expectations that collider bounds could be more promising when DM direct detection scattering is suppressed, for example, by kinematics due to (very) light DM mass, or due to non-coherent WIMP-nucleus SD interactions. Nevertheless, with this good result, the authors of [144] have mentioned that an introduction of a light mediator significantly weakens the collider bounds. Furthermore, any direct detection discovery that is in apparent conflict with obtained mono-jet limits will thus point to a new light state coupling the SM to the dark sector [144]. Some suspicions appear after these words concerning usefulness of the Tevatron constraints for direct detection experiments. For example, if one plans a new direct DM search experiment with ambitious goals, he should take into account the already achieved results, especially those, which teach one where is nothing to see. The Tevatron limits excluded some regions of DM properties, but, nevertheless, the authors of the results openly allowed a direct DM experiment to look for signal in the excluded region.

Most complete theoretically justified investigations of the collider limits for the DM properties were given in [143, 145]. Models, where a WIMP DM candidate $\chi$ is a fermion or a scalar interacting with quarks and/or gluons, were analysed within the EFT. The authors assumed that the WIMP is the only new particle in the energy ranges relevant for current experiments. (It looks like a very strong assumption, because in almost all reliable BSM theories it is not true). Next WIMPs appear in pair only. Under this assumption, the WIMP will couple to the SM particles through higher dimensional operators in the EFT, presumably mediated by particles of the dark sector which are somewhat heavier than the WIMP itself. The WIMP is assumed to be a singlet under the SM gauge groups, and thus possesses no tree-level couplings to the electroweak gauge bosons.

Contrary to only 4 effective operators discussed in [144] a complete list of 24 operators was considered in [145]. The operators for Dirac fermions and scalars fall into six categories (Table 1) with characteristic $E_{\mathrm{T}}^{\text {miss }}$ spectral shapes [153]. For each operator the parameter $M_{*}$ can be determined (or constrained) from comparison of (initiated by this operator) WIMP-pair hadroproduction $(p p, p \bar{p} \rightarrow \chi \chi+X)$ with relevant measurements.

This set of high dimensional contact operators defines the Effective Field Theory (EFT) description of the WIMP-hadron interactions. It is a nonrenormalizable field theory and it breaks down at some energy scale represented by the masses of those particles which have been integrated out. In the operator definitions, $M_{*}$ is the suppression scale of the heavy mediator particles that are integrated out. The quantities $M_{*}$ which characterize the interaction strength of the interactions are functions of the masses and the coupling strengths of the mediating particles to WIMPs and SM fields and can be computed in terms of the fundamental parameters for any full-scale theory [145]. 
TABLE 1. Operators coupling WIMPs, denoted as $\chi$, to SM particles. The operator names beginning with D, C, R apply to WIMPs that are Dirac fermions, complex scalars or real scalars respectively. From [145].

\begin{tabular}{|l|c|l|c|l|c|}
\hline Name & Operator & Name & Operator & Name & Operator \\
\hline D1 & $m_{q} \bar{\chi} \chi \bar{q} q / M_{*}^{3}$ & D9 & $\bar{\chi} \sigma^{\mu \nu} \chi \bar{q} \sigma_{\mu \nu} q / M_{*}^{2}$ & $\mathrm{C} 3$ & $\chi^{\dagger} \partial_{\mu} \chi \bar{q} \gamma^{\mu} q / M_{*}^{2}$ \\
D2 & $i m_{q} \bar{\chi} \gamma^{5} \chi \bar{q} q / M_{*}^{3}$ & $\mathrm{D} 10$ & $i \bar{\chi} \sigma_{\mu \nu} \gamma^{5} \chi \bar{q} \sigma_{\alpha \beta} q / M_{*}^{2}$ & $\mathrm{C} 4$ & $\chi^{\dagger} \partial_{\mu} \chi \bar{q} \gamma^{\mu} \gamma^{5} q / M_{*}^{2}$ \\
D3 & $i m_{q} \bar{\chi} \chi \bar{q} \gamma^{5} q / M_{*}^{3}$ & $\mathrm{D} 11$ & $\alpha_{s} \bar{\chi} \chi G_{\mu \nu} G^{\mu \nu} / 4 M_{*}^{3}$ & $\mathrm{C} 5$ & $\alpha_{s} \chi^{\dagger} \chi G_{\mu \nu} G^{\mu \nu} / 4 M_{*}^{2}$ \\
$\mathrm{D} 4$ & $m_{q} \bar{\chi} \gamma^{5} \chi \bar{q} \gamma^{5} q / M_{*}^{3}$ & $\mathrm{D} 12$ & $i \alpha_{s} \bar{\chi} \gamma^{5} \chi G_{\mu \nu} G^{\mu \nu} / 4 M_{*}^{3}$ & $\mathrm{C} 6$ & $i \alpha_{s} \chi^{\dagger} \chi G_{\mu \nu} \tilde{G}^{\mu \nu} / 4 M_{*}^{2}$ \\
$\mathrm{D} 5$ & $\bar{\chi} \gamma^{\mu} \chi \bar{q} \gamma_{\mu} q / M_{*}^{2}$ & $\mathrm{D} 13$ & $i \alpha_{s} \bar{\chi} \chi G_{\mu \nu} \tilde{G}^{\mu \nu} / 4 M_{*}^{3}$ & $\mathrm{R} 1$ & $m_{q} \chi^{2} \bar{q} q / 2 M_{*}^{2}$ \\
$\mathrm{D} 6$ & $\bar{\chi} \gamma^{\mu} \gamma^{5} \chi \bar{q} \gamma_{\mu} q / M_{*}^{2}$ & $\mathrm{D} 14$ & $\alpha_{s} \bar{\chi} \gamma^{5} \chi G_{\mu \nu} \tilde{G}^{\mu \nu} / 4 M_{*}^{3}$ & $\mathrm{R} 2$ & $\chi^{2} \bar{q} \gamma^{5} q i m_{q} / 2 M_{*}^{2}$ \\
$\mathrm{D} 7$ & $\bar{\chi} \gamma^{\mu} \chi \bar{q} \gamma_{\mu} \gamma^{5} q / M_{*}^{2}$ & $\mathrm{C} 1$ & $m_{q} \chi^{\dagger} \chi \bar{q} q / M_{*}^{2}$ & $\mathrm{R} 3$ & $\alpha_{s} \chi^{2} G_{\mu \nu} G^{\mu \nu} / 8 M_{*}^{2}$ \\
$\mathrm{D} 8$ & $\bar{\chi} \gamma^{\mu} \gamma^{5} \chi \bar{q} \gamma_{\mu} \gamma^{5} q / M_{*}^{2}$ & $\mathrm{C} 2$ & $i m_{q} \chi^{\dagger} \chi \bar{q} \gamma^{5} q / M_{*}^{2}$ & $\mathrm{R} 4$ & $i \alpha_{s} \chi^{2} G_{\mu \nu} \tilde{G}^{\mu \nu} / 8 M_{*}^{2}$ \\
\hline
\end{tabular}

The authors focused on the mono-jet event search at the Tevatron, where the WIMPs recoil against a single jet $(\bar{p} p \rightarrow \chi \chi+j+X)$, with restrictions on any additional SM radiation. These mono-jet searches were used to determine the constraints on the coefficients of the effective operators as a function of the WIMP mass. Many relevant figures are given in [145]. The bounds on the strength of effective-operator interactions of WIMPs with hadrons were translated into constraints on the possible contributions to direct detection cross sections for each of those operators in the form of numerous exclusion curves (for each operator from Table 1). In the $\sigma_{\mathrm{DM}}-m_{\mathrm{DM}}$ planes (Figs. 78 ) these curves are superimposed with the relevant direct detection exclusion curves for comparison (and competition).

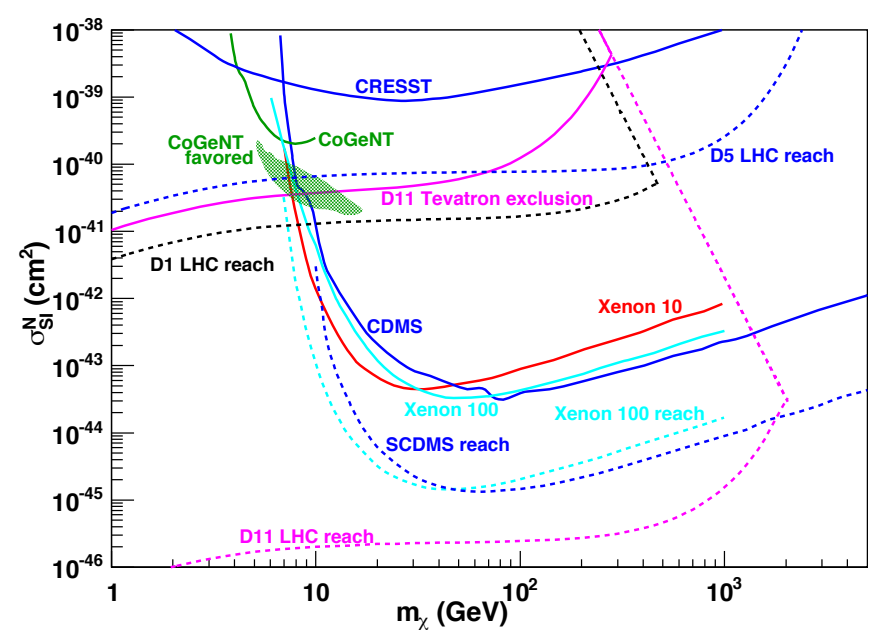

FiguRe 7. Experimental limits on spin-independent WIMP direct detection from several direct DM search experiments [154, 155, 156, 157, 158, 159, 160] in comparison with the Tevatron exclusion curves (for the operator D11 - solid magenta line) and LHC discovery reaches (dashed lines) for relevant operators. Figure from [145].

As authors [145] noted, in all cases colliders can probe regions of very light WIMP masses more effectively than direct detection experiments. Furthermore, it was shown that for many considered operators the direct detection rates are expected to be very small because of the 


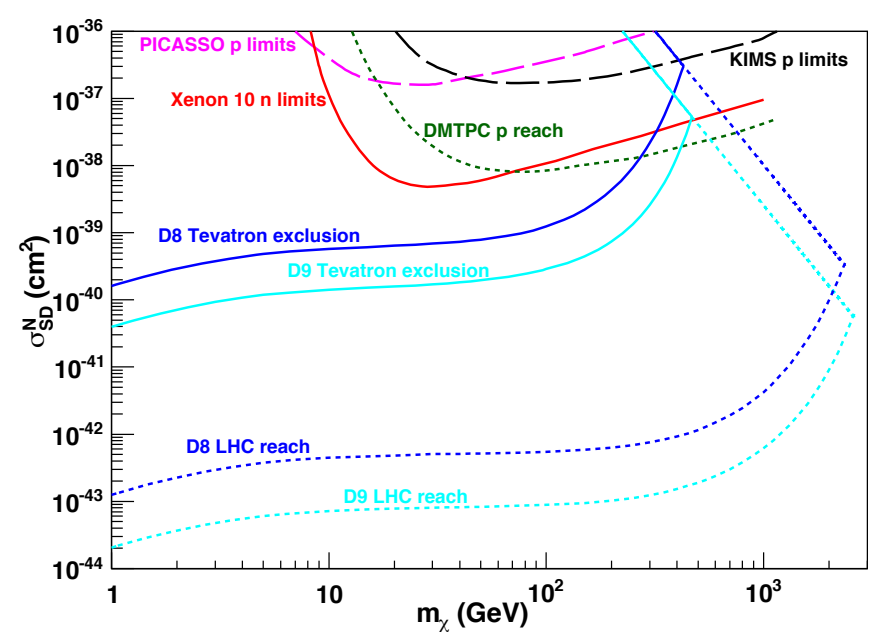

FiguRE 8. Experimental limits on spin-dependent WIMP direct detection from several direct DM search experiments [161, 162, 156, 163] in comparison with the Tevatron exclusions curves (solid lines) and LHC discovery reaches (dashed lines) for relevant operators. Figure from [145].

velocity suppression, and colliders become the only way to effectively probe such kind of possible WIMP-hadron interactions. The question concerning applicability of these operators for true DM interaction still remained unanswered. In the case of a WIMP whose dominant recoil is through a SD interaction, collider constraints are already much stronger even than the expected reaches of near-future direct detection experiments.

The important question on validity of the EFT approach was discussed already in [145]. Obviously, the validity of EFT itself and what happens above the regime of its validity depends on the underlying complete model, and therefore is inevitably model dependent. The specifics of a complete model makes collider bounds stronger or weaker. In some sense the fact reincarnates the model-dependence in the usage of EFT. To allow the EFT approach, some lower bound for any $M_{*}$ should exist depending on a given WIMP mass. These constraints are shown in Figs. 78 as straight lines for large $m_{\chi}$.

People believe [164] the EFT approach allows a valuable comparison of complementary results on DM detection. If any of the experiments sees a signal, the interpretation in this approach can lead to further insights into the nature of DM as well as the underlying physics by a comparison of different techniques and observables.

In general, the EFT approach is considered conservative, but in regimes where the validity might be questioned the cross section is mostly underestimated, compared to the full theory, which leads to more conservative limits [145]. Finally, the authors wrote that while effective theories may not always capture our favorite parameters of our favorite complete models, they do provide a language to describe WIMP-SM interactions which captures a wide class of theories in a fairly model-independent fashion [145].

A reader could ask - for what? Being sooner or later detected, the DM particle must be implemented into one of our (perhaps, new) "favorite complete models" like, for example, SUSY. It is not clear how this "fairly model-independent fashion" could help. 
Concluding this section one can first say that the competition (comparison) between direct DM and collider DM search experiments has started to run only in the field of exclusion curves. Unfortunately this competition helps almost nothing on the way to real DM detection (section 3). Next, one can point out that a huge bulk of publications on the subject appeared after the famous papers [143, 144, 145]. A start of the "direct" DM search at the LHC was given, followed by many proposals for very exotic DM searches with other accelerators, and not only (section 7).

And finally, speaking about DM search or ever detection with a collider one must not forget that a WIMP is not yet a DM particle. As nicely mentioned recently in [22, 61, 13, the discovery of a DM signal at particle colliders only establishes the production of a particle with lifetime greater than about $100 \mathrm{~ns}$. The assumption that this particle contributes to DM requires an extrapolation in lifetime of 24 orders of magnitude! It is only by corroborating a particle collider discovery through another method that one can claim that the collider discovery is relevant for cosmology. Colliders cannot say anything about the stability of WIMPs which is the essential property of the true DM particle [165]. Therefore only the direct DM detection can play the decisive role in the DM problem (section 8).

\section{ATLAS RESUlts on the DM SEARCH AT LHC}

As already discussed in section 5 , the missing large transverse energy, $E_{\mathrm{T}}^{\mathrm{miss}}$, (caused by the escaping GeV-scale-mass WIMPs) is the key signature on which the idea of the LHC search for DM particles strongly relies. The events possessing the $E_{\mathrm{T}}^{\text {miss }}$ can be produced in association with ordinary matter (which tag the event) - photons, jets (from quarks or gluons), $W-, Z-$, Higgs-bosons and heavy quarks ( $b$ - and top-quark). One assumes these SM particles back-toback recoil against the undetected WIMPs, making the latter "visible" due to a large value of measured $E_{\mathrm{T}}^{\mathrm{miss}}$. More generally, these events (with large $E_{\mathrm{T}}^{\mathrm{miss}}$ ) constitute a low-background sample that provides powerful sensitivity to new physics phenomena [166, 167].

Despite the point that the LHC experiments cannot establish whether a WIMP candidate is stable on cosmological time scales and hence is a true DM candidate the terms WIMP and DM-particle are used as synonymous [151] in all experimental papers in the section.

The first ATLAS paper on "direct" Search for dark matter candidates and large extra dimensions in events with a photon and missing transverse momentum in pp collision data at $\sqrt{s}=7$ TeV with the ATLAS detector was published at the very beginning of 2013 [166]. New phenomena were looked for in events with an energetic photon and large missing transverse momentum $\left(p p \rightarrow \gamma+E_{\mathrm{T}}^{\text {miss }}+X\right)$ at $7 \mathrm{TeV}$ and integrated luminosity of $4.6 \mathrm{fb}^{-1}$. The measurements were found to be in good agreement with the SM predictions for the background (Fig. 9). The obtained results were converted into model-independent 90\% CL upper limits on the visible (new physics) cross section $\sigma \times A \times \epsilon$ (cross section $\times$ acceptance $\times$ efficiency) of $5.6 \mathrm{fb}$. The results were further translated into exclusion curves on the pair-production cross section of WIMP candidates in $p p \rightarrow \chi \chi+\gamma+X$ under the assumption that the pair can be traced (optimistically, detected) due to energy imbalance with an energetic photon (from initial-state radiation). The generic graph in Fig. 10 shows typical production of two WIMPs in a collision of quarks from initial protons [165]. 


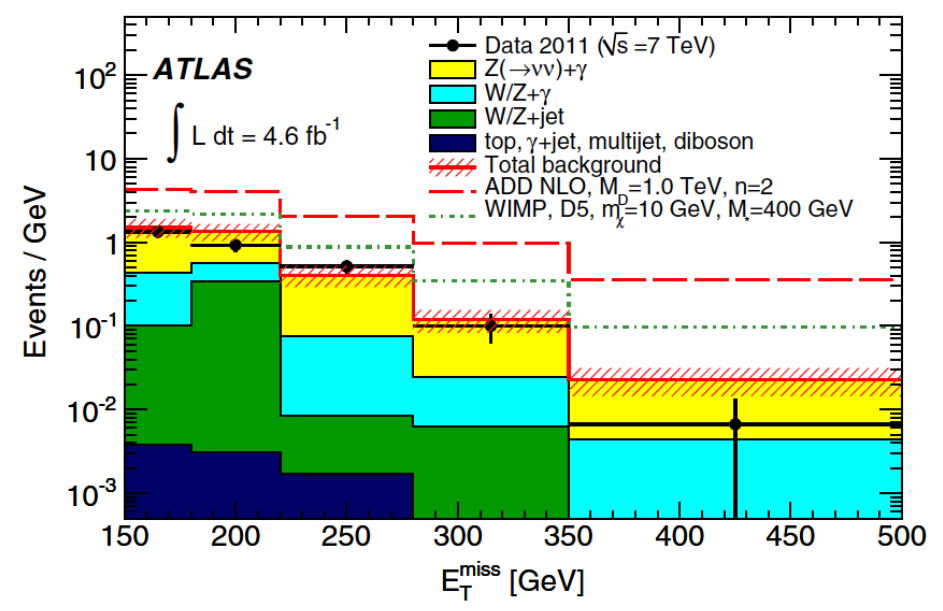

Figure 9. Measured $E_{\mathrm{T}}^{\text {miss }}$ distribution in comparison with the relevant SM background predictions and some expectations. From [166].

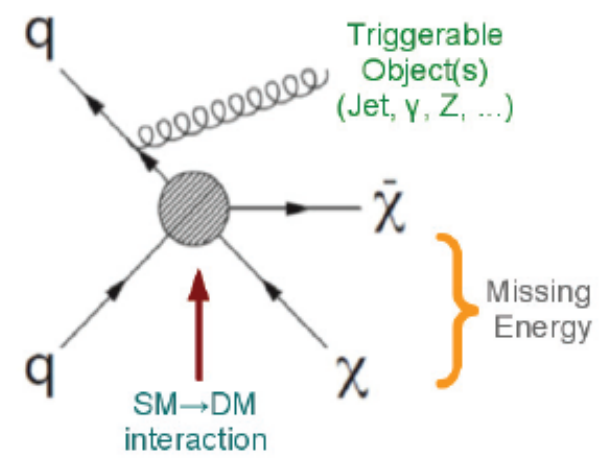

Figure 10. Production of WIMP candidates tagged by a measurable object from initialstate radiation. The final state monojet and/or monophoton together with (large) $E_{\mathrm{T}}^{\text {miss }}$ provides a new physics collider signature. From [165].

To obtain the limits on WIMP-SM interaction one needs a tool to describe interaction of WIMPs with the SM particles. To this end the EFT approach with several operators from [145] was used. The WIMPs were assumed to be Dirac fermions with $m_{\chi}$ between $1 \mathrm{GeV} / c^{2}$ and $1.3 \mathrm{TeV} / c^{2}$ and the effective operators D1 (scalar), D5 (vector), D8 (axial-vector), and D9 (tensor) from Table 1 were used. These operators correspond to spin-independent (D1 and D5) and spin-dependent (D8 and D9) interactions. In the case of the D1 (D5) spin-independent operator, values of $M_{*}$ below 31 and $5 \mathrm{GeV}$ (585 and $156 \mathrm{GeV}$ ) were excluded at 90\% CL for $m_{\chi}$ equal to $1 \mathrm{GeV} / c^{2}$ and $1.3 \mathrm{TeV} / c^{2}$, respectively. Values of $M_{*}$ below 585 and $100 \mathrm{GeV}$ (794 and $188 \mathrm{GeV}$ ) were excluded for the D8 (D9) spin-dependent operator for $m_{\chi}$ equal to $1 \mathrm{GeV} / c^{2}$ and $1.3 \mathrm{TeV} / c^{2}$, respectively. On the basis of the prescription of [145] these results were translated into exclusion curves - upper limits for the nucleon-WIMP interaction cross section as a function of $m_{\chi}$ (Fig. 11). From the figure one can conclude [166] that obtained results (under validity of the EFT) gave the best exclusion limits for spin-independent nucleonWIMP interactions in the small-mass region $\left(1<m_{\chi}<3.5 \mathrm{GeV} / c^{2}\right)$ and for spin-dependent 




Figure 11. Upper limits (90\% CL) on the nucleon-WIMP cross section as a function of WIMP mass for spin-dependent (left) and spin-independent (right) interactions. The results are compared with previous mono-jet and mono-photon results at colliders [151, [167, 168] and results from direct detection experiments. From [166].

interactions for all masses $\left(1 \mathrm{GeV} / c^{2}<m_{\chi}<1 \mathrm{TeV} / c^{2}\right)$. These results confirmed the general expectations (discussed in section 5), and improved previous CDF-based achievements.

The second ATLAS paper on Search for dark matter candidates and large extra dimensions in events with a jet and missing transverse momentum with the ATLAS detector was published in the middle of 2013 [151]. Again, new phenomena were searched in events with a high-energy jet and large missing transverse momentum $\left(p p \rightarrow\right.$ jet $\left.+E_{\mathrm{T}}^{\text {miss }}+X\right)$ using data at $7 \mathrm{TeV}$ and an integrated luminosity of $4.7 \mathrm{fb}^{-1}$.

The main physics results of the search are given in Fig. 12 as the measured $E_{\mathrm{T}}^{\text {miss }}$ and leading jet $p_{\mathrm{T}}$ distributions in comparisons with the SM predictions. No excess of events beyond expectations from SM processes was observed and some limits on the visible cross sections of new physics processes were obtained.

The EFT approach (section 5) was used for comparison of the LHC DM-search results with the results of direct and indirect DM searches. According to Table 1, interactions of SM particles and WIMPs are described by only two parameters, the scale $M_{*}$ and the WIMP particle mass $m_{\chi}$. From measured distributions (Fig. 12) lower limits on the mass parameters $M_{*}$ of effective operators associated with the above-mentioned new processes can be first derived as functions of the WIMP mass $m_{\chi}$ [164]. To this end, under assumptions of WIMP pair-production and absence of any other possible new particles, 5 effective WIMP-SM contact operators from Table 1 were considered, and for each of them constraints on $M_{*}$ as functions of $m_{\chi}$ were derived and presented in Fig. 4 of paper [151].

Next, these bounds on $M_{*}$ (for a given $m_{\chi}$ ) can be converted within EFT into the bounds on WIMP-nucleon cross sections, which are probed by direct DM detection experiments (section 3). Depending on the type of interaction, contributions to SD- or SI- WIMP-nucleon interactions 

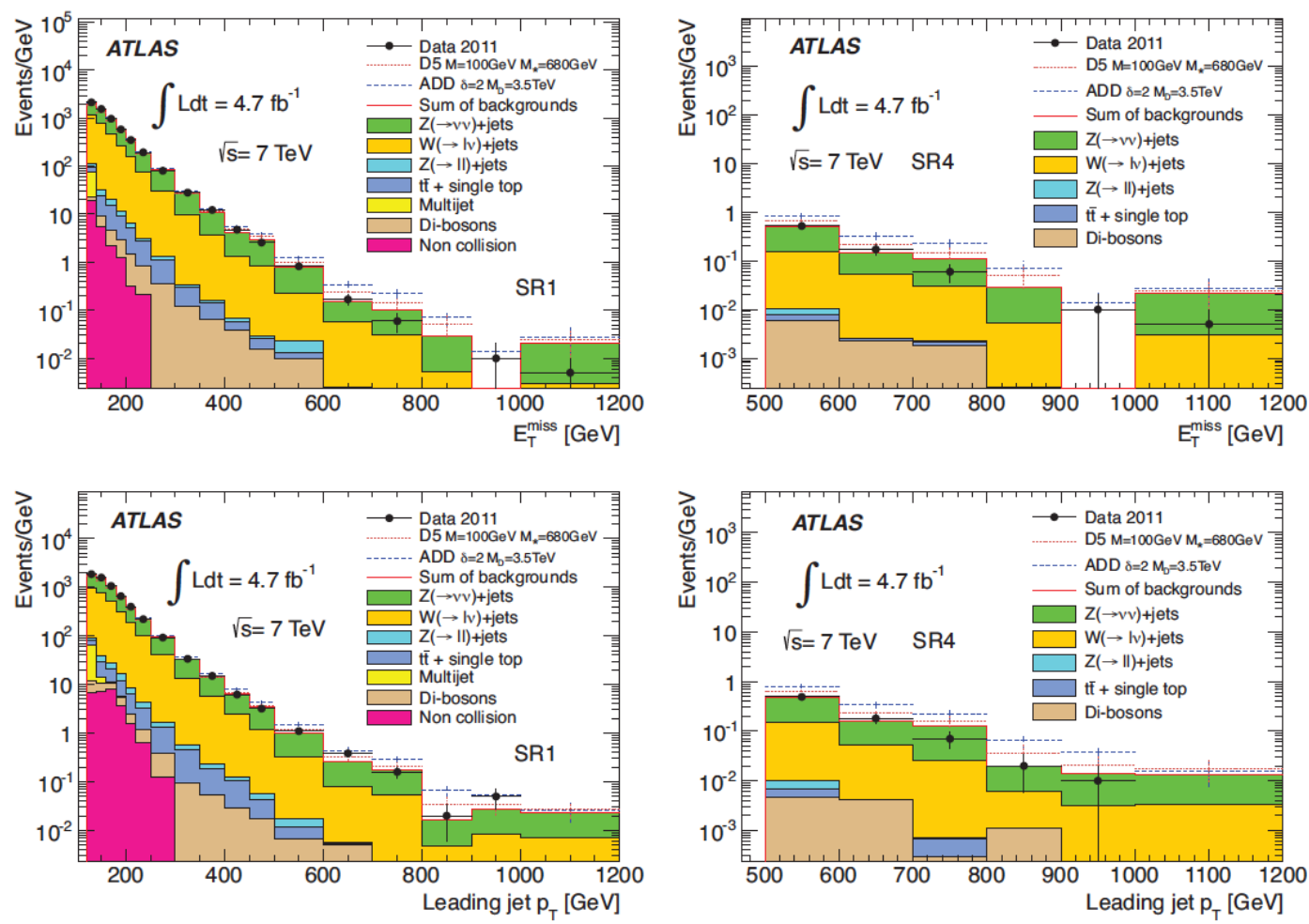

FIGURE 12. Measured $E_{\mathrm{T}}^{\text {miss }}$ and leading jet $p_{\mathrm{T}}$ distributions in comparison with the relevant SM (background) predictions. From [151].

are expected. This translation into bounds on WIMP-nucleon cross sections is shown in Fig. 13 [151. Again, the spin-independent ATLAS-based exclusion curves are particularly relevant in
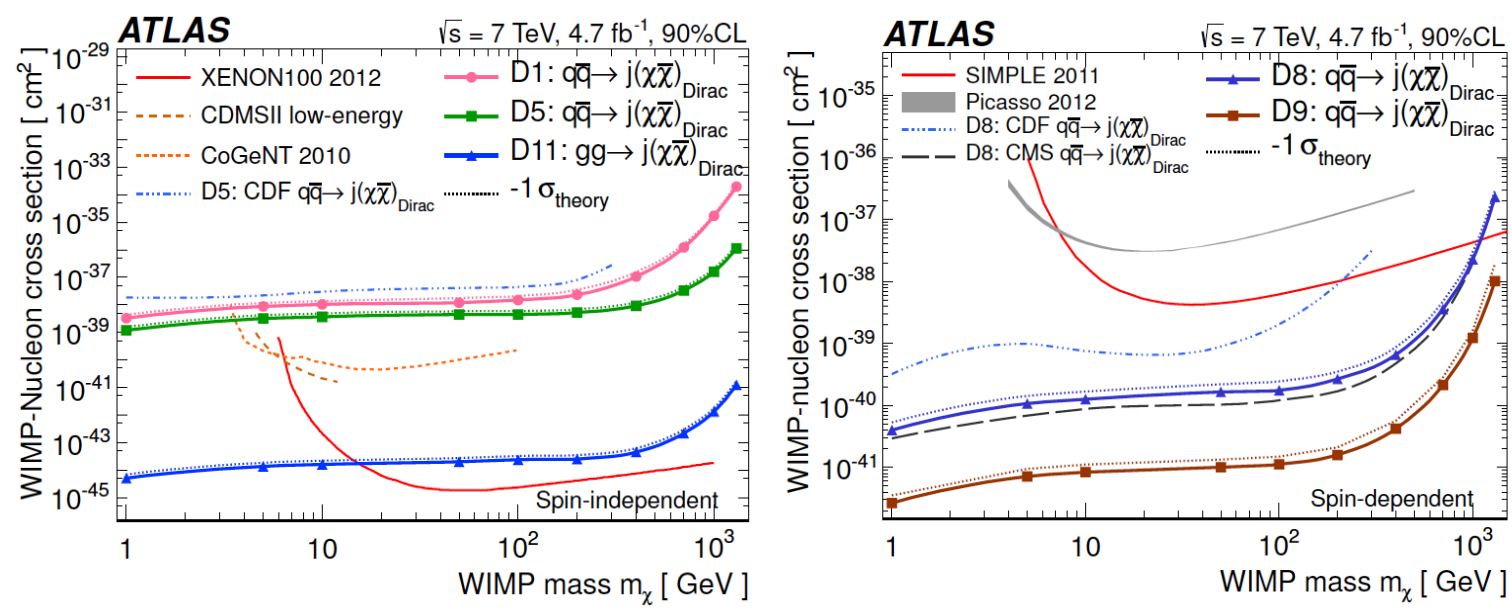

Figure 13. ATLAS limits (90\% CL) on spin-independent (left) and spin-dependent (right) WIMP-nucleon scattering cross sections. More details are in [151].

the low $m_{\chi}$ region $\left(<10 \mathrm{GeV} / c^{2}\right)$ where the direct DM limits could suffer from a technical problem with a very low recoil energy deposition. The bound looks "especially powerful" in the case of the gluon D11-operator, it is competitive to direct detection bounds up to $m_{\chi}$ of $20 \mathrm{GeV} / c^{2}$, provided this gluon operator is relevant for the direct DM search technique. Some 
of the limits are substantially better than the limits set by direct and indirect DM detection experiments, in particular at small WIMP masses $m_{\chi}<10 \mathrm{GeV} / c^{2}$ [151].

The EFT approach [145] also allows one to interpret the obtained collider limits in terms of the relic abundance of WIMPs, or WIMP self-annihilation rate, which is defined as the product of the annihilation cross section times the relative velocity, averaged over the DM velocity distribution. This interpretation was shown in Fig. 7 of [151], where the limits on vector and axial-vector effective operators were translated into upper limits on the WIMP annihilation rate to the four light quark flavours. The complementarity between collider and indirect searches for DM was demonstrated by the figure [151]. Nevertheless, it is important to remember [165] this complementarity remains valid or makes sense only under a number of important assumptions - the EFT must be valid, WIMPs must interact with SM particles exclusively via only one of the EFT operators (since a mix of operators with potential interference effects is not considered), and the interactions must be flavour-universal for the four light quarks.

In general, the ATLAS mono-jet results are somewhat better than the ATLAS mono-photon one overall due to the higher statistics in the data samples [165].

The 3rd ATLAS paper about Search for dark matter in events with a hadronically decaying $W$ or $Z$ boson and missing transverse momentum ... was published on 29 January 2014 [169] and already used data at $8 \mathrm{TeV}$ and $20.3 \mathrm{fb}^{-1}$. Events with a hadronic jet with the jet-mass consistent with a $W$ - or $Z$-boson, and with large missing transverse momentum were analyzed. For the first time, the goal was to look for the presence for a WIMP pair as DM candidates $(\chi \bar{\chi})$, produced in $p p$ collisions in association with a $W$ or $Z$ boson $\left(p p \rightarrow W / Z+E_{\mathrm{T}}^{\text {miss }}+X\right.$, see Fig. 14). As before, one assumed that the undetected $\chi \bar{\chi}$-pair produces large missing transverse
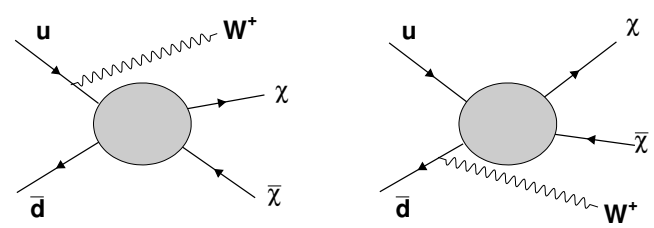

Figure 14. General graph for pair production of WIMPs $(\chi \bar{\chi})$ in $p p$ collisions with initial-state radiation of a $W$ boson. The same graphs can be given for initial-state radiation of a $Z$ boson. From [169].

momentum ( $E_{\mathrm{T}}^{\text {miss }}>150 \mathrm{GeV}$ in [169]), which can be "detected" (balanced) by means of a single massive jet reconstructed from the hadronic decays $W \rightarrow q \bar{q}^{\prime}$ or $Z \rightarrow q \bar{q}$.

The proposal to use the signature with hadronical decays of $W$ - or $Z$-boson is based on the results of [170], where a source of enhancement was found. Usually, due to a large rate of gluon/quark initial-state radiation relative to $\gamma$-, $W$ - or $Z$-boson radiation, the strongest limits mainly come from mono-jet analyses. But the EFT operators used in the mono-jet search (Table 1) have equal couplings of the $\chi$-particles to $u$-type and $d$-type quarks. From Fig. 14 one can see two ways of the $W$-boson radiation - from the initial $u$-quark or $\bar{d}$-quark. In the case of equal coupling, this interference is destructive and gives a small $W$-boson emission rate. Otherwise, due to constructive interference, the mono- $W$-boson production can be strongly enhanced [170] in comparison with other possibilities (moon-jet, mon-photon, etc). 
After sophisticated analysis, the finally obtained results and predicted backgrounds in the two signal regions are shown in Fig. 15 for the $m_{\text {jet }}$ distribution. The data agreed well with the

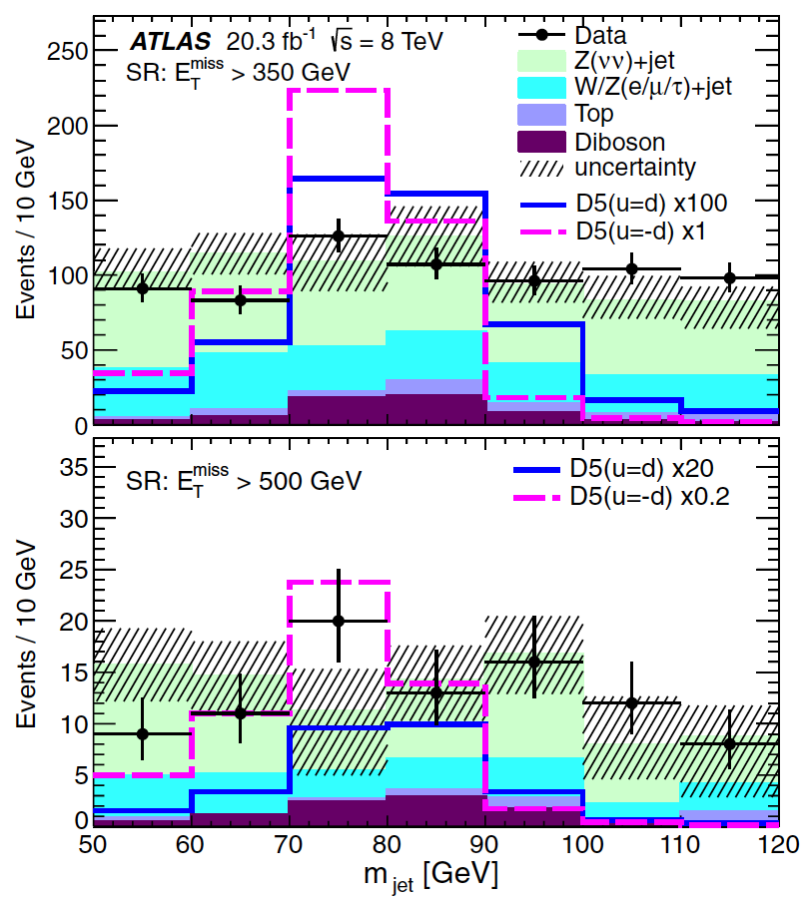

FiguRE 15. Distribution of $m_{\text {jet }}$ in the data and for the background in the signal regions (SR) with $E_{\mathrm{T}}^{\text {miss }}>350 \mathrm{GeV}$ (top) and $E_{\mathrm{T}}^{\text {miss }}>500 \mathrm{GeV}$ (bottom). Also shown are the combined mono- $W$-boson and mono- $Z$-boson signal distributions with $m_{\chi}=1 \mathrm{GeV} / c^{2}$ and $M_{*}=1 \mathrm{TeV}$ for the D5 destructive and D5 constructive cases. From [169].

background SM estimate for each $E_{\mathrm{T}}^{\text {miss }}$ threshold.

On the basis of this result new limits (as a function of $m_{\chi}$ ) were set on the mass scales $M_{*}$ of the C1 (scalar), D1 (scalar), D5 (vector), and D9 (tensor) effective operators from Table 1 . Figure 16 shows the excluded regions in the $M_{*}-m_{\chi}$ plane for these operators. These limits were set on the WIMP DM signals using the expected shape of the "signal" $m_{\text {jet }}$ distribution given in Fig. 15. It is remarkable, how much these $M_{*}$-limits differ from each other for different

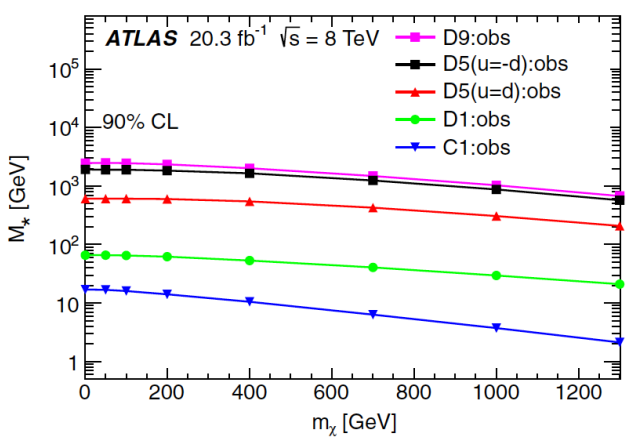

FiguRE 16. Observed limits on the effective theory mass scale $M_{*}$ as a function of $m_{\chi}$ at $90 \%$ CL from combined mono- $W$-boson and mono- $Z$-boson signals for various operators. For each operator, the values below the corresponding line are excluded. From [169]. 
operators.

In Figure 17 for both the spin-independent (C1, D1, D5) and the spin-dependent interaction (D9) exclusion curves for the WIMP-nucleon cross sections are given following method of [145]. The results are traditionally compared with measurements from direct detection experiments.

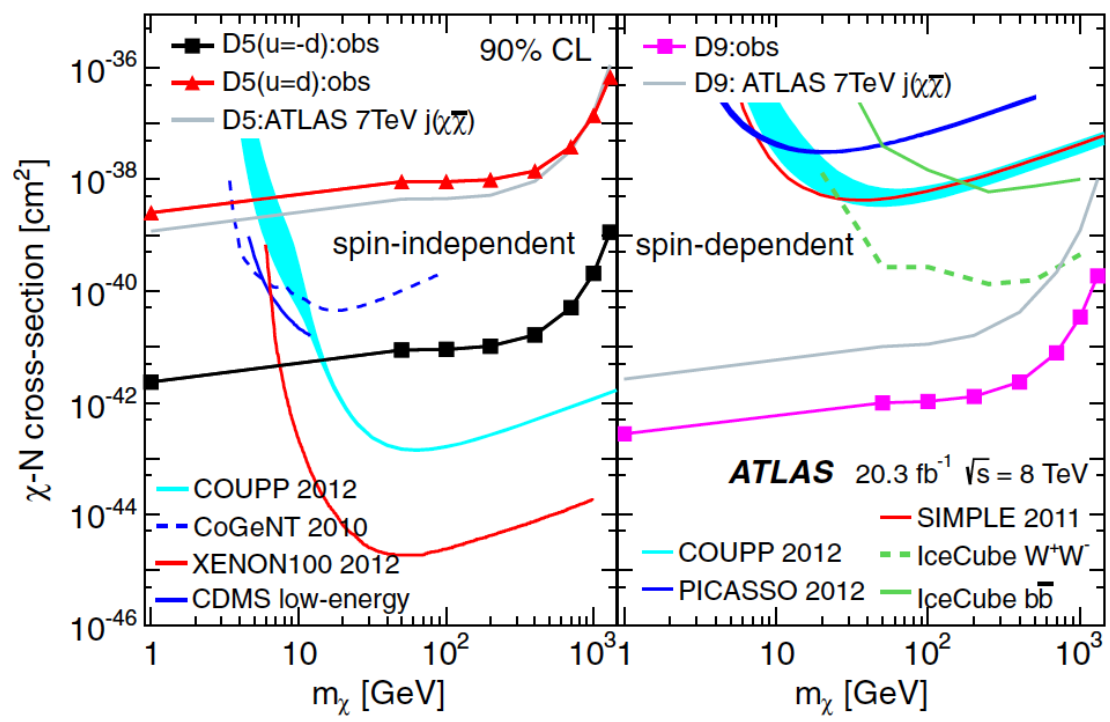

Figure 17. Limits on $\chi$-nucleon cross sections as a function of WIMP mass $m_{\chi}$ at $90 \%$ CL for spin-independent (left) and spin-dependent (right) effective operators, compared to some previous limits [70, 171, 157, 87, 91, 172, 173]. From [169].

One can conclude from this figure [169] that (as generally expected for a collider DM search) the search for WIMP pair production in association with $W / Z$-boson extends the limits on the WIMP-nucleon cross section in the low mass region $m_{\chi}<10 \mathrm{GeV} / c^{2}$. Next, the comparison of these new limits with the limits set by ATLAS in the $7 \mathrm{TeV}$ mono-jet analysis demonstrates improvement by about 3 orders of magnitude. The reason is the constructive interference (due to the opposite sign of $u \chi$-type and $d \chi$-type couplings), which has led to a very large increase in the $W$-boson-associated production cross section. For other cases, the limits are similar.

Assuming a simple model of WIMP production via the Higgs boson, a re-analysis of the data [169] was carried out. The upper limit on the Higgs boson production through $W H$ and $Z H$ modes and decay to invisible particles is obtained to be $1.3 \mathrm{pb}$ at $95 \%$ CL for $m_{H}=125$ $\mathrm{GeV} / c^{2}$. Figure 18 shows the upper limit of the total cross section of $W H$ and $Z H$ processes with $H \rightarrow \chi \bar{\chi}$, normalized to the SM next-to-leading order prediction for the $W H$ and $Z H$ production cross section $\left(0.8 \mathrm{pb}\right.$ is predicted for $m_{H}=125 \mathrm{GeV} / c^{2}$ in $\left.\mathrm{SM}\right)$, which is 1.6 at $95 \%$ CL for $m_{H}=125 \mathrm{GeV} / c^{2}$. This was the first new experimental result on the subject.

Continuation of the ATLAS collaboration search for DM with the help of the $Z$ and Higgs bosons was carried out in the dedicated paper [174]. Here the $E_{\mathrm{T}}^{\mathrm{miss}}$ was used as a signal - the momentum of the reconstructed $Z$ boson is expected to be balanced by the momentum of the invisibly decaying Higgs boson (with its mass allowed in the range $110<m_{H}<400 \mathrm{GeV} / c^{2}$ ). The measured $E_{\mathrm{T}}^{\text {miss }}$-distribution in events with an electron or a muon pair consistent with $Z$ boson decay can be used to constrain the $Z H$ production cross section times the branching ratio of the Higgs boson decaying to invisible particles. 


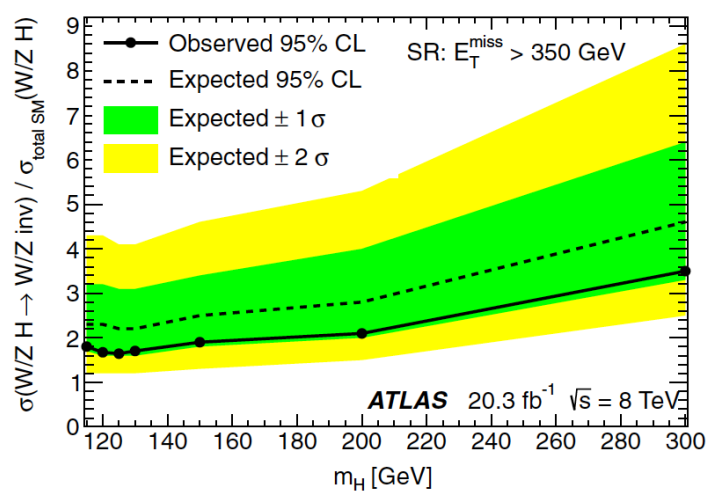

Figure 18. Limit on the Higgs boson cross section for decay to invisible particles divided by the cross section for decays to SM particles as a function of $m_{H}$ at $95 \% \mathrm{CL}$, derived from the signal region (SR) with $E_{\mathrm{T}}^{\mathrm{miss}}>350 \mathrm{GeV}$. From [169].

The total cross section for the associated production of the SM Higgs boson, with $m_{H}=$ 125.5 GeV $/ c^{2}$, and a $Z$ boson, according to [175] is $331 \mathrm{fb}$ at $\sqrt{s}=7 \mathrm{TeV}$ and $410 \mathrm{fb}$ at $\sqrt{s}=$ $8 \mathrm{TeV}$. The branching ratio of the Higgs boson decay to invisible SM particles $\left(H \rightarrow Z Z^{*} \rightarrow 4 \nu\right)$ is $1.2 \times 10^{-3}$. The performed search could not be sensitive to this value, and the main idea was to look for some enhancement in the invisible decay mode due to a contribution of not-SM particles.

The numbers of observed and expected events are shown in Table 2, Figure 19 shows the

TABLE 2. Number of events observed in data and expected from the signal $\left(m_{H}=125.5\right.$ $\mathrm{GeV} / c^{2}, \sigma_{Z H}^{\mathrm{SM}}, \mathrm{BR}(H \rightarrow$ invisible $\left.)=1\right)$ and from each background source for the 7 and 8 TeV data-taking periods. From [174].

\begin{tabular}{|l|c|c|}
\hline Data Period & $2011(7 \mathrm{TeV}+4.5 / \mathrm{fb})$ & $2012(8 \mathrm{TeV}+20.3 / \mathrm{fb})$ \\
\hline$Z Z \rightarrow \ell \ell \nu \nu$ & $20.0 \pm 0.7 \pm 1.6$ & $91 \pm 1 \pm 7$ \\
$W Z \rightarrow \ell \nu \ell \ell$ & $4.8 \pm 0.3 \pm 0.5$ & $26 \pm 1 \pm 3$ \\
Dileptonic $t \bar{t}, W t, W W, Z \rightarrow \tau \tau$ & $0.5 \pm 0.4 \pm 0.1$ & $20 \pm 3 \pm 5$ \\
$Z \rightarrow e e, Z \rightarrow \mu \mu$ & $0.13 \pm 0.12 \pm 0.07$ & $0.9 \pm 0.3 \pm 0.5$ \\
$W+$ jets, multijet, semileptonic top & $0.020 \pm 0.005 \pm 0.008$ & $0.29 \pm 0.02 \pm 0.06$ \\
Total background & $25.4 \pm 0.8 \pm 1.7$ & $138 \pm 4 \pm 9$ \\
\hline Signal & $8.9 \pm 0.1 \pm 0.5$ & $44 \pm 1 \pm 3$ \\
\hline Observed & 28 & 152 \\
\hline
\end{tabular}

$E_{\mathrm{T}}^{\text {miss }}$ distribution after the full event selection for the $8 \mathrm{TeV}$ data and the expected backgrounds. No significant deviation from the SM expectation was observed in both sets of data collected at $7 \mathrm{TeV}$ and $8 \mathrm{TeV}$ by the ATLAS experiment.

Assuming the SM rate for $Z H$ production, an upper limit of $75 \%$ (at 95\% CL) was set on the branching ratio of the SM Higgs boson decay to invisible particles. The expected limit in the absence of beyond-SM decays to invisible particles is $62 \%$ (at 95\% CL). Limits were also set on the cross section times branching ratio for additional neutral Higgs boson, with $110<m_{H}<400$ $\mathrm{GeV} / c^{2}$, produced in association with a $Z$ boson and decaying to invisible particles. 


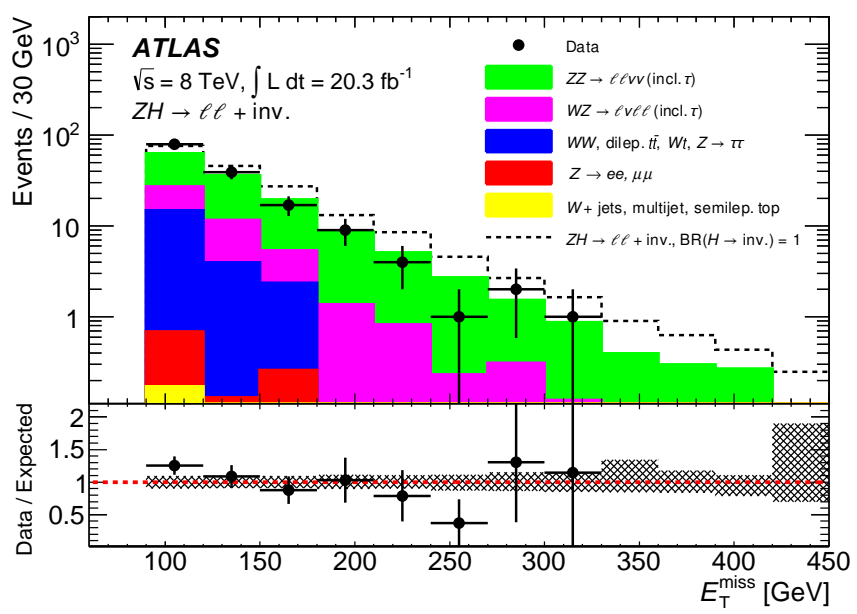

Figure 19. Distribution of $E_{\mathrm{T}}^{\text {miss }}$ after the full selection in the $8 \mathrm{TeV}$ data (dots). The filled stacked histograms represent the background expectations. The signal expectation for Higgs boson with $m_{H}=125.5 \mathrm{GeV} / c^{2}$, a SM $Z H$ production rate and $\mathrm{BR}(H \rightarrow$ invisible) $=1$ is stacked on top of the background expectations. From [174].

The obtained limit on $\mathrm{BR}(H \rightarrow$ invisible $)$ for the $125-\mathrm{GeV}$ Higgs boson can be interpreted in terms of an upper limit on the WIMP-nucleon cross section, when the Higgs boson serves as a mediator between WIMP and SM particles and therefore can decay to the WIMP pair [146]. The formalism used to interpret the $\mathrm{BR}(H \rightarrow$ invisible) limit in terms of the spin-independent WIMP-nucleon cross sections is described in [176, 177].

Figure 20 shows upper limits on the WIMP-nucleon cross section for three cases in which a single WIMP candidate was considered, being a scalar, a vector or a Majorana fermion. There

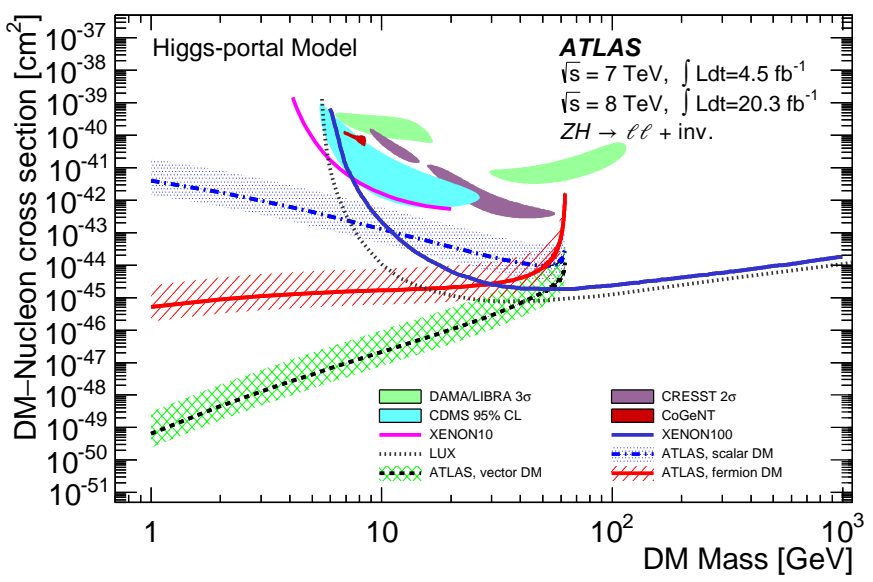

FigURE 20. Limits on the WIMP-nucleon scattering cross section (90\% CL), extracted from the $\mathrm{BR}(H \rightarrow$ invisible) limit, compared to results from direct-search experiments. Spin-independent results from direct-search experiments are shown from $85,178,70,79$, [18, 179, 180, 73]. From [174]. 
is no sensitivity to these models once the mass of the WIMP candidate exceeds $m_{H} / 2$. The Higgs-nucleon coupling was taken as $0.33_{-0.07}^{+0.30}$ [177], the uncertainty of which is expressed by the bands in the figure. It is seen that one has [174 the strongest limits for low-mass WIMP DM candidates.

The next step of the ATLAS DM program was Search for dark matter in events with a $Z$ boson and missing transverse momentum at $\sqrt{s}=8 \mathrm{TeV} \ldots$ [181]. Events with large $E_{\mathrm{T}}^{\mathrm{miss}}$ and $e^{+} e^{-}$- or $\mu^{+} \mu^{-}$-pairs consistent with the decay of a $Z$ boson (Fig. 21) were analyzed at 20.3 $\mathrm{fb}^{-1}$ statistics of $8-\mathrm{TeV}$ data. It was assumed that the large $E_{\mathrm{T}}^{\text {miss }}$ stemmed from the escaping

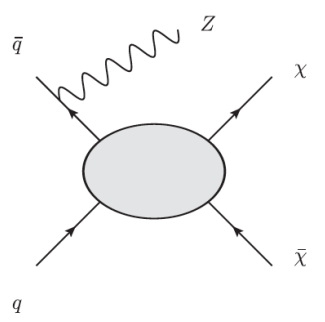

(a)

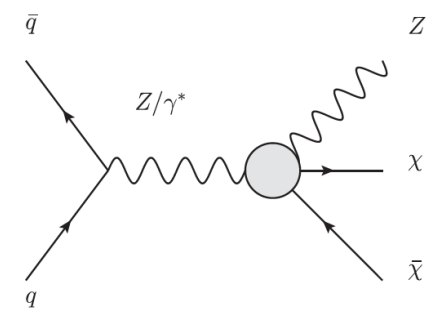

(b)

Figure 21. Graph (a) shows 2 WIMPs and $Z$ boson production $p p \rightarrow \chi \bar{\chi}+Z$ with the ISR operator, and graph (b) shows the same process via the $Z Z \chi \chi$ vertex. From [181.

$\chi \bar{\chi}$ particles. Several signal regions with different requirements on the $E_{\mathrm{T}}^{\text {miss }}$ were defined. From Fig. 22 one can conclude that no excess above the SM prediction (the background) was observed. This is the main model-independent result of [181].

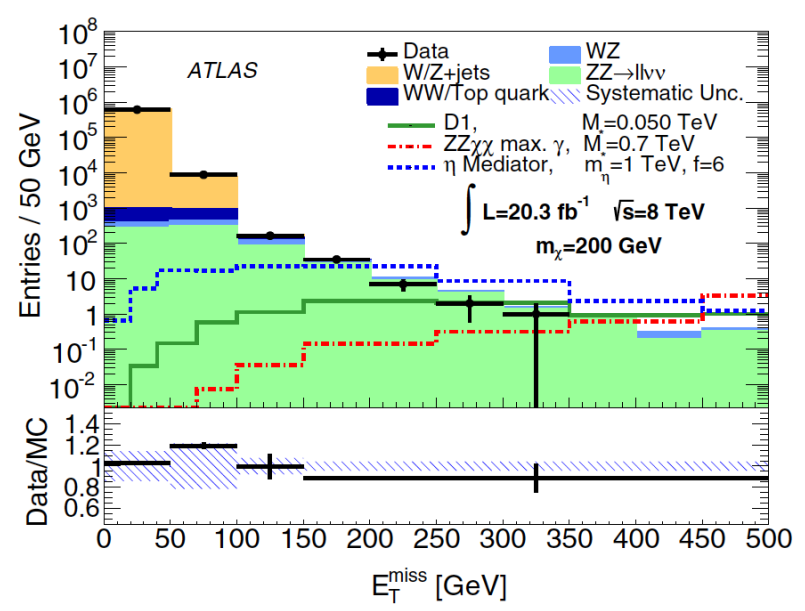

Figure 22. $\quad E_{\mathrm{T}}^{\text {miss }}$ distributions after all event selections. The hypothetical $p p \rightarrow Z \chi \bar{\chi}$ signals are given for various values of the mass scale, $M_{*}$. The WIMP mass is $m_{\chi}=200$ $\mathrm{GeV} / c^{2}$. From [181]

For interpretation of the obtained results the EFT framework [145] recently extended to describe interactions with electroweak bosons [146] was used. Again, the $\chi$-particle is considered as the only new particle; the mediator mass is assumed to be larger than typical momentum transfer, and the WIMPs are produced in pairs only. For graph (a) in Fig. 21 one used contact operators D1, D5, D9 from Table 1. Following [146], dimension-5 and dimension-7 operators 
were used to describe the WIMP interaction with the electroweak bosons. The dimension7 operator couples $\chi$ to $Z \gamma^{*}$ and $\chi$ to $Z Z$. Since a $Z$ boson is in the final state for each operator, intermediate states with a $Z$ or $\gamma^{*}$ each contribute to the matrix element. The relative contribution of the $Z$ and $\gamma^{*}$ diagrams is a parameter of the theory. According to Fig. 21, two models of the WIMP $+Z$ production were considered, via initial state $Z$-boson radiation, and when $Z$ interacts directly with WIMPs. The latter case was not previously investigated.

The $E_{\mathrm{T}}^{\text {miss }}$ region (Fig. 22 with the best expected limit on the cross section for $p p \rightarrow Z \chi \bar{\chi}+X$ production was used to calculate the limits (given in Fig. 23) on the mass scale $M_{*}$ as a function of $m_{\chi}$ for each effective operator in both above-mentioned models. Again, one sees substantial

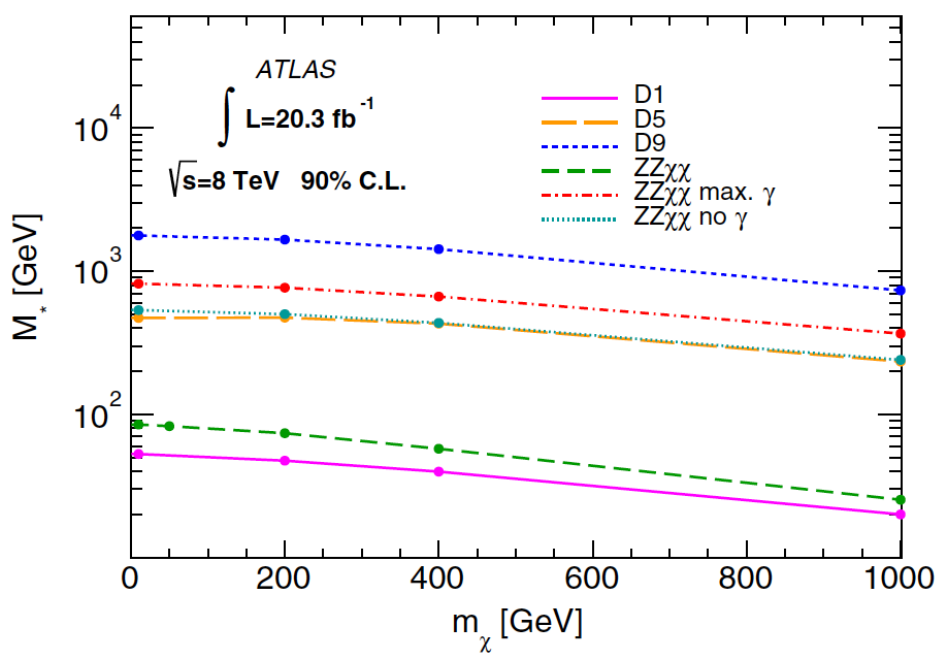

Figure 23. Lower limits (90\% CL) on the mass scale, $M_{*}$, of considered six contact operators as a function of $m_{\chi}$. For each operator, the values below the corresponding line are excluded. From [181].

variation of the $M_{*}$ as a function of the form of the effective operators.

To complete the traditional "collider-DM-search" analysis one transformed EFT limits from Fig. 23 into exclusion curves for the $\chi$-nucleon cross sections. The comparison of these limits with the direct and indirect detection exclusion curves is shown in Figs. 24 and 25. One can conclude from these figures that the ATLAS spin-dependent limits under discussion are less stringent than the ATLAS limits for WIMP candidates recoiling against a $W$ or $Z$ boson decaying to hadrons [169]. The limits degrade by $13-23 \%$, depending on the $E_{\mathrm{T}}^{\text {miss }}$ signal region under consideration. On the contrary, there is some improvement between the ATLAS results in the case of the spin-independent $\chi$-nucleon interaction, but, in general, all collider limits are still far to be competitive with direct DM limits.

In [181 limits are also set on the coupling and mediator mass of a model in which the interaction is mediated by a scalar particle [182]. In this model a scalar-mediator $\eta$, with mass $m_{\eta}$, and a $\eta$-WIMP coupling strength $f$ is responsible for the production of the DM particles. Limits on the cross section times branching ratio in the scalar-mediator model are shown in Fig. 7 of [181], and limits on $f$ as a function of mediator mass $m_{\eta}$ and $m_{\chi}$, as well as the exclusion region, are shown in Fig. 8 of [181]. These limits do not look very exciting, and neither does their further applicability. 


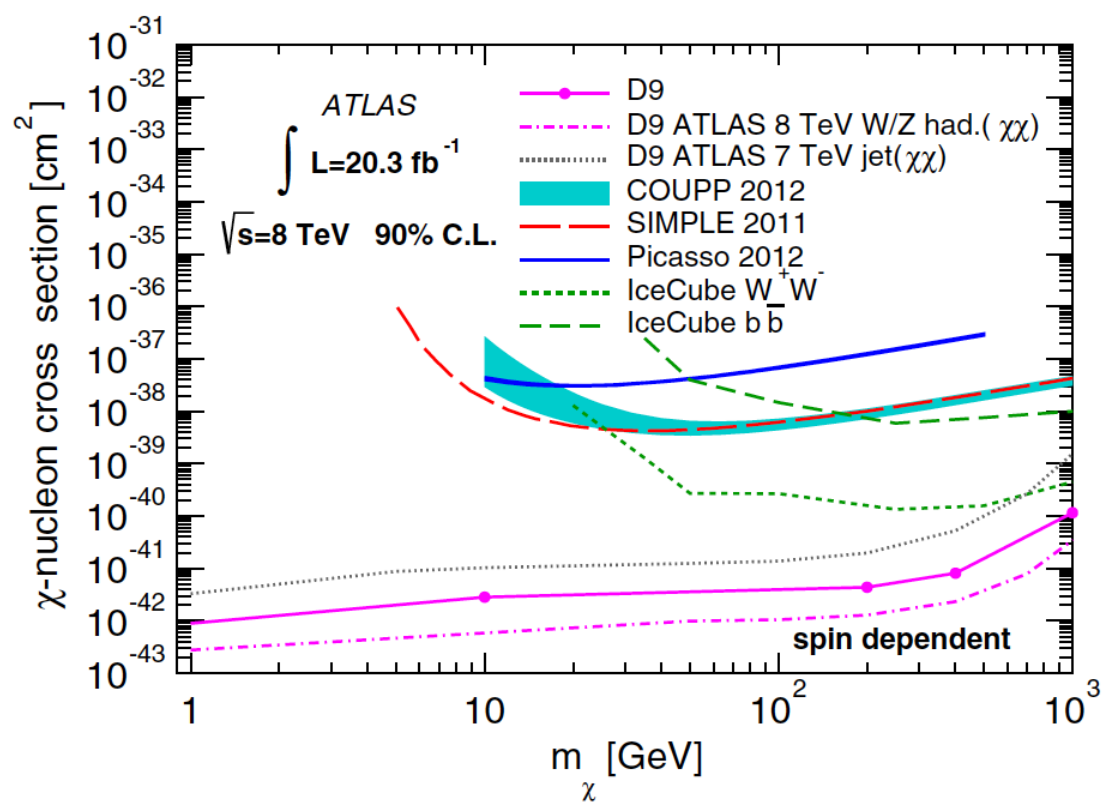

Figure 24. Observed upper limits $(90 \% \mathrm{CL})$ on the $\chi$-nucleon cross section as a function of $m_{\chi}$ for the spin-dependent D9 operator. The limits are compared with ATLAS results from hadronically decaying $W / Z$ [169] and $j+\chi \chi$ [151] searches, COUPP [173], SIMPLE [91, PICASSO [87, and IceCube [172]. From [181.

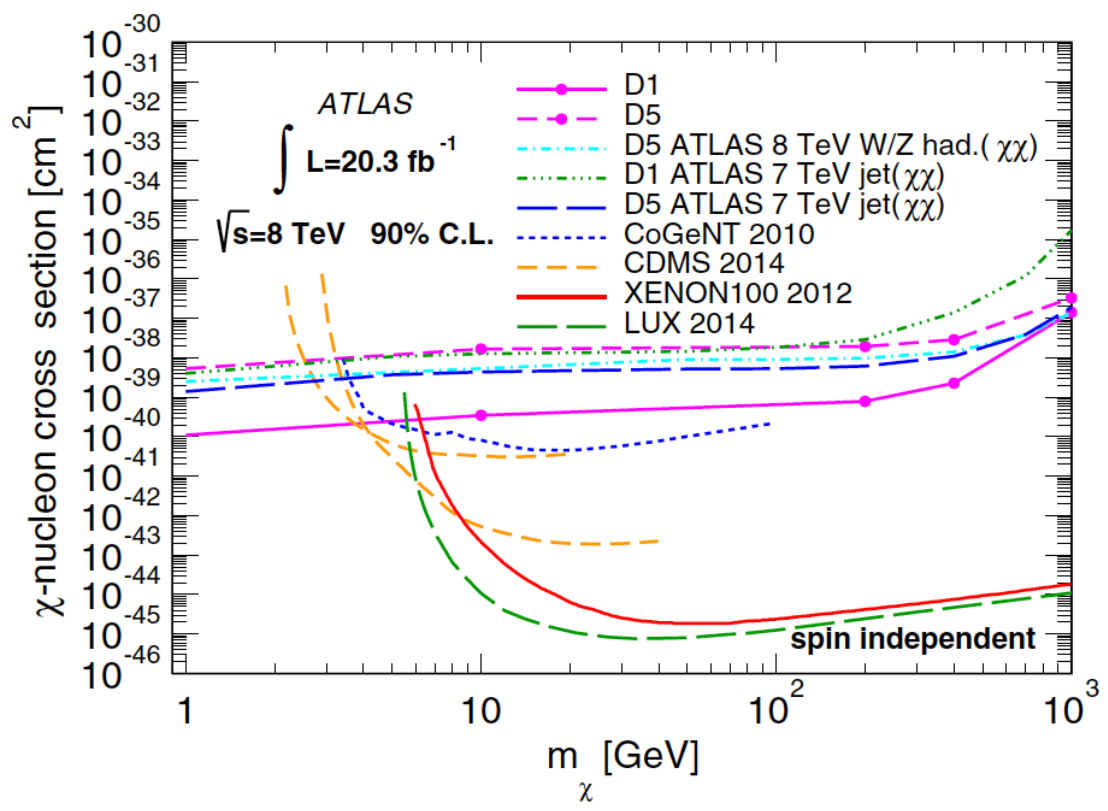

Figure 25. Observed upper limits $(90 \% \mathrm{CL})$ on the $\chi$-nucleon cross section as a function of $m_{\chi}$ for spin-independent effective operators. The limits are compared with ATLAS results from hadronically decaying $W / Z$ [169] and $j+\chi \chi$ [151] searches, CoGeNT [157, XENON100 [70, CDMS [180, 82, and LUX 73]. From [181].

Investigation of the DM problem with the ATLAS detector was continued by dedicated Search for dark matter in events with heavy quarks and missing transverse momentum ... [183] with $20.3 \mathrm{fb}^{-1}$ of $p p$ collisions collected at $8 \mathrm{TeV}$. This search for WIMP pair production in association 
with bottom or top quarks $(p p \rightarrow \chi \chi+b(\bar{b}), t(\bar{t})+X)$ was carried out in events with large $E_{\mathrm{T}}^{\text {miss }}$ produced together with high-momentum jets of which at least one was a b-quark-jet (Fig. 26). Final states with top quarks were selected by requiring a high jet multiplicity and in some cases
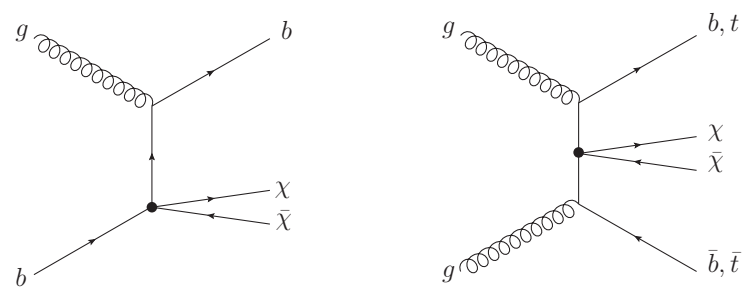

FiguRE 26. Dominant graphs for WIMP $\chi$ production in conjunction with (left) a single $b$-quark and (right) a heavy quark (bottom or top) pair. From [183].

a single lepton [183].

Figure 27 shows the measured $E_{\mathrm{T}}^{\mathrm{miss}}$ distributions for three signal regions and the so-called Razor variable $R$-distribution for the 3 rd signal region. Variable $R$ allowed one to utilize both transverse and longitudinal information about the event [184]. On this basis maximal rejection of the abundant $t \bar{t}$-background was achieved for $R>0.75$. In the figure all expected signals of
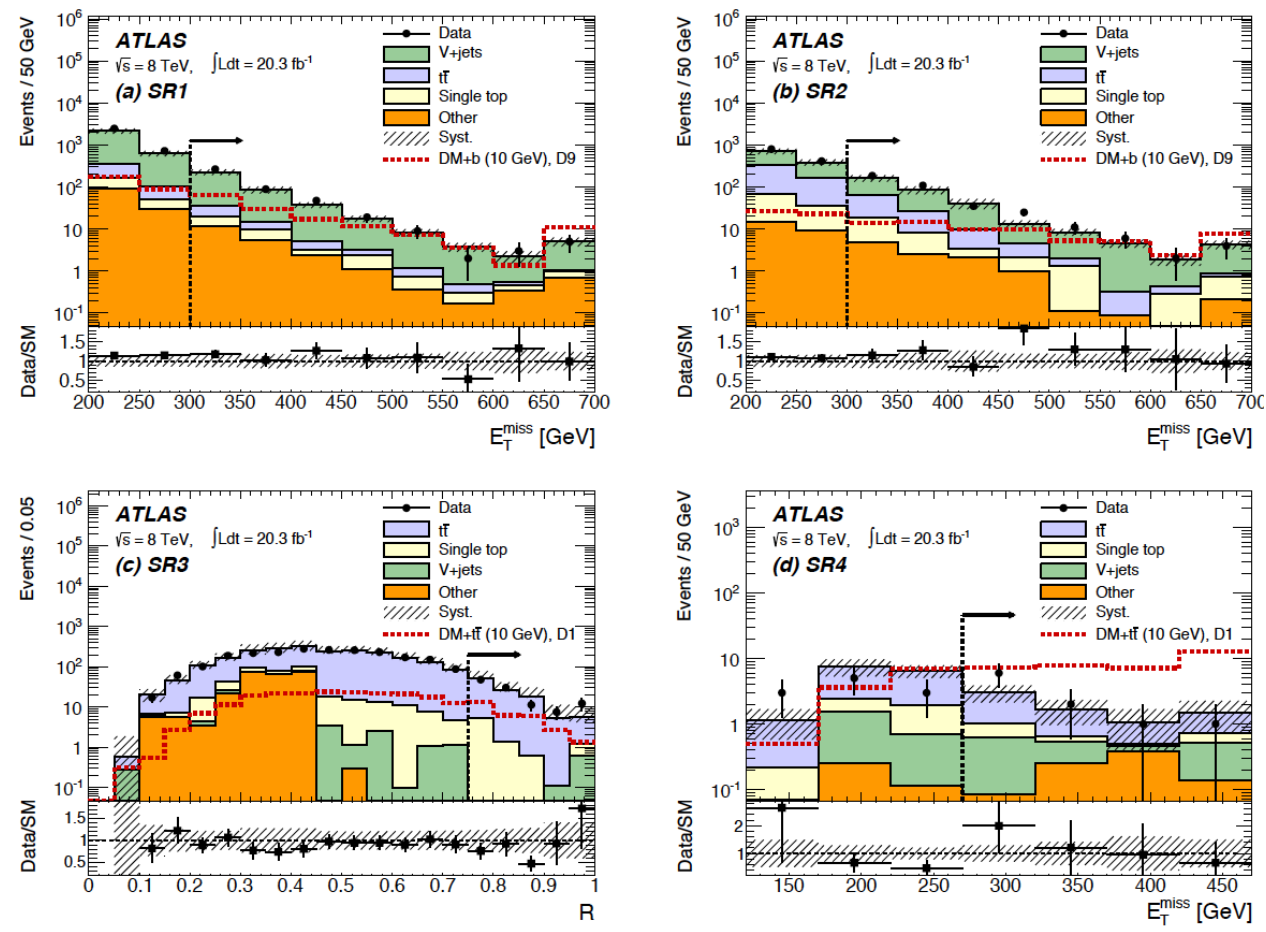

Figure 27. Comparison between data and expected SM background. $E_{\mathrm{T}}^{\text {miss }}$ distributions in SR1 (a) and SR2 (b) with expected signal (red line) for $\chi \bar{\chi}+b(\bar{b})$ due to D9operator. $R$ distribution in SR3 (c) excluding the selection on $R$. $E_{\mathrm{T}}^{\text {miss }}$ distribution in SR4 (d) excluding the selection on $E_{\mathrm{T}}^{\text {miss }}$. In (c), (d) the expected signal (in red) is given for $\chi \bar{\chi}+t \bar{t}$ due to D1-operator. The final selection requirements are indicated by an arrow. Other backgrounds are composed of diboson and multijet production. From [183].

the WIMP production were calculated for $m_{\chi}=10 \mathrm{GeV} / c^{2}$. The data distributions were found to be consistent with the relevant SM expectations. 
From the measured $E_{\mathrm{T}}^{\text {miss }}$-distributions limits were set on the mass scale $M_{*}$ of the scalar D1, C1 and tensor D9 EFT operators (Table 1), that were used to describe WIMP-SM interactions [145]. For these operators Fig. 28 shows $M_{*}-m_{\chi}$ lower limits obtained from 4 different signal regions SR1-SR4, defined in Table 1 of [183]. Typical rather substantial $M_{*}$-dependence on a
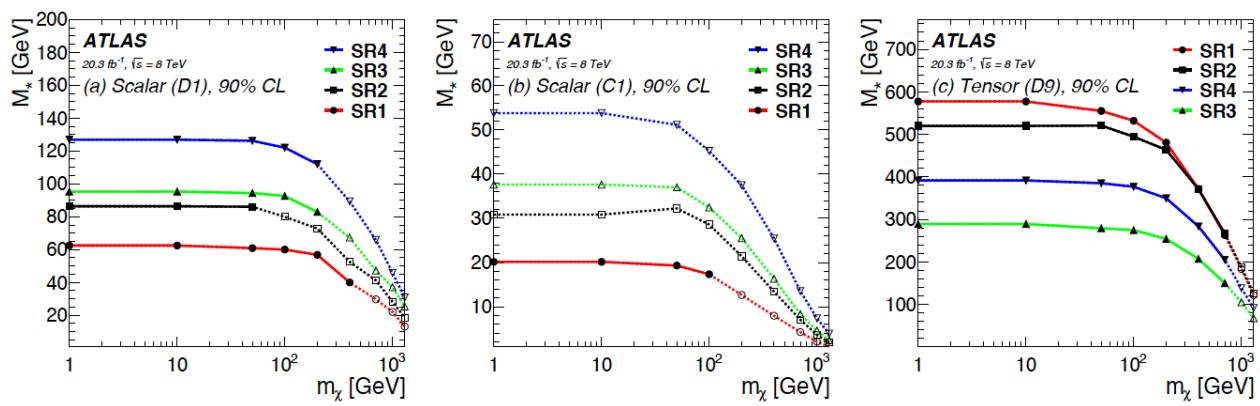

FiguRE 28. Lower limits on $M_{*}(90 \%$ CL) for the SR1 (red), SR2 (black), SR3 (green), and SR4 (blue) as a function of $m_{\chi}$ for the operators (a) D1, (b) C1, and (c) D9. Solid lines and markers indicate the validity range of the EFT. the dashed lines and hollow markers represent the full collider constraints. From [183.

type of EFT operator and signal region is clearly seen.

From Fig. 28 the traditional exclusion curves for the WIMP-nucleon cross-section for SI and SD interactions were obtained. Figure 29 shows that, as expected, the limits on the $\chi$-nucleon
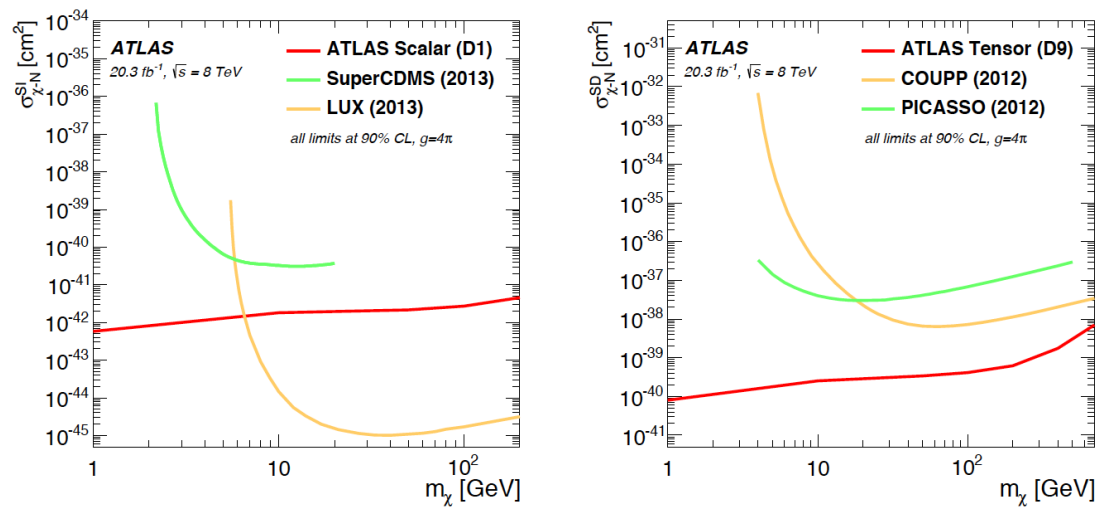

FiguRE 29. 90\% CL upper limits (red) on the $\chi$-nucleon cross-section as a function of $m_{\chi}$ (left) for the spin-independent coupling (scalar operator D1), and (right) for the spindependent coupling (tensor operator D9). The other curves show the exclusion curves from some of direct DM experiments [73, 180, 173, 87]. From 183.

cross-section improve rather significantly previous constraints in the regions of low-mass WIMPs.

The results of Fig. 27 were also interpreted in a bottom-Flavoured DM model (b-FDM) [185]. The model was proposed to explain the Fermi-LAT excess of $\gamma$-rays from the Galactic center in terms of specific DM annihilation, when the DM particles with mass of about $35 \mathrm{GeV} / c^{2}$ annihilated into $b$-quarks via a colored new scalar field, $\phi$ (Fig. 30). The DM $\chi$ particle is assumed to be a Dirac fermion that couples to right-handed down-type quarks, mainly to the $b$-quarks. Therefore the collider signature is $b$-quarks produced in association with $E_{\mathrm{T}}^{\text {miss }}$. 


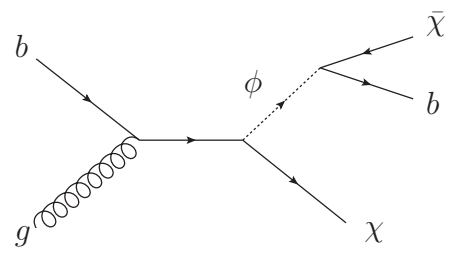

Figure 30. Example of DM, $\chi$, production in the $b$-FDM model. From [183].

From the observed exclusion contour in the $\left(m_{\chi}, m_{\phi}\right)$ plane given in Fig. 7 of [183], one concluded that in the $b$-FDM model mediators with $300<m_{\phi}<500 \mathrm{GeV} / c^{2}$ are excluded at $95 \%$ CL for DM particles with $m_{\chi} \simeq 35 \mathrm{GeV} / c^{2}$. Unfortunately, this information is not yet enough to reject completely the $b$-FDM model. The general conclusion of [183] is typical — the data are consistent with the SM, limits are the strongest at low DM masses.

The ATLAS collaboration has produced new results of mono-photon, mono-lepton and monojet study with $8 \mathrm{TeV}$ data, and has improved previously published 7-TeV-based DM constraints. Remarkably, the new "8-TeV" papers used "new phenomena" or "new particles" instead of the words "dark matter" in their titles.

Results of a search for new phenomena in events with an energetic photon and large missing transverse momentum in $p p$ collisions at $8 \mathrm{TeV}$ and integrated luminosity of $20.3 \mathrm{fb}^{-1}$ were reported in [186]. The obtained $E_{\mathrm{T}}^{\text {miss }}$ distribution of events with an energetic photon is shown in Fig. 31. The observed (in signal region) 521 events were well described by the SM background

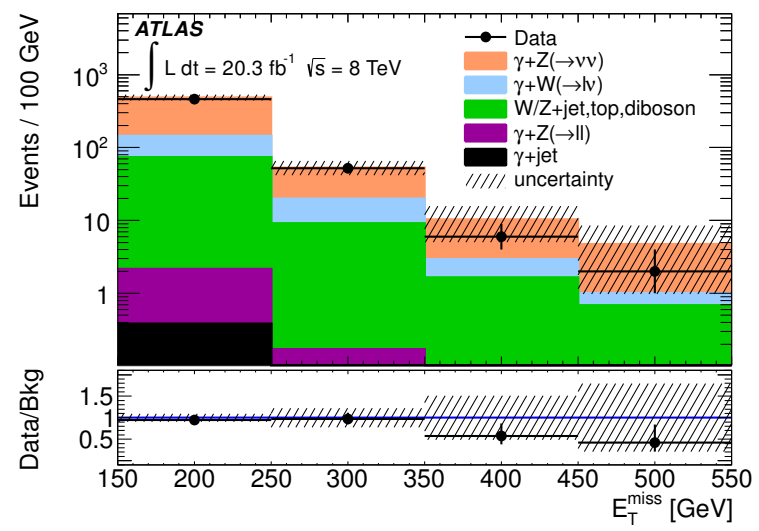

Figure 31. Distribution of $E_{\mathrm{T}}^{\text {miss }}$ in the signal region for the data and for the background. The lower part of the figure shows the ratios of the data to the expectedbackground event yields. From [186].

prediction of $557 \pm 36 \pm 27$, extrapolated from control regions. These results were interpreted in terms of exclusions on models that would produce an excess of the $\gamma+E_{\mathrm{T}}^{\text {miss }}$ events.

If $\sigma$ denotes a cross section of a new physics process, producing the $\gamma+E_{\mathrm{T}}^{\text {miss }}$ signature, then the most model-independent limit can be set on the fiducial cross section $\sigma \times A$. The fiducial acceptance $A$ was defined in [186]. The limit on $\sigma \times A$ was derived from a limit on the visible cross section $\sigma \times A \times \epsilon$, where $\epsilon$ is the fiducial reconstruction efficiency. A conservative estimate $\epsilon=69 \%$ was computed using WIMP samples with no quark/gluon produced from the main interaction vertex. The finally observed upper limit on the fiducial cross section was $5.3 \mathrm{fb}(95 \%$ 
CL). This limit is applicable to any model that produces $\gamma+E_{\mathrm{T}}^{\mathrm{miss}}$ events in the fiducial region and has similar reconstruction efficiency $\epsilon$ [186].

In [186] both the EFT approach [145] with two parameters $m_{\chi}$ and $M_{*}$ and a model with a $Z^{\prime}$-like mediator [146] were considered. In the latter case the mediator state $V$ can be explicitly produced when the typical momentum transfer in LHC collisions could reach the scale of the microscopic interaction $Q \geq m_{V}$ (Fig. 32). The interaction is described in the model by four

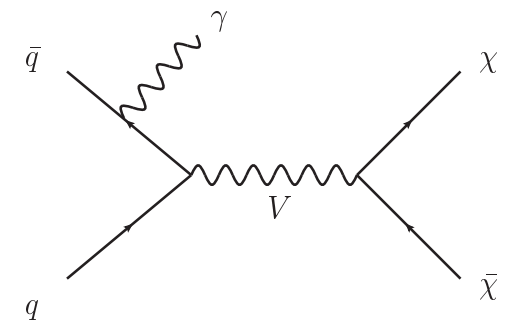

Figure 32. Production of $\chi \bar{\chi}$-pairs via an explicit $s$-channel $V$-mediator. The mass suppression scale of contact interaction appears via $M_{*}=m_{V} / \sqrt{g_{f} g_{\chi}}$, where $m_{V}$ is the mediator mass, $g_{f}$ and $g_{\chi}$ represent the relevant coupling factors. From [186].

parameters $-m_{\chi}, m_{V}$, the width of the mediator $\Gamma$, and the overall coupling $\sqrt{g_{f} g_{\chi}}$.

From the main results given in Fig. 31 traditional EFT constraints were derived in the form of limits on the $M_{*}$ as a function of $m_{\chi}$. But at this time one has cared about validity of the EFT. When the momentum transfer $Q$ becomes comparable to the mass of the intermediate state $m_{V}=M_{*} \sqrt{g_{f} g_{\chi}}$ the EFT approach fails [145, 148]. In order to have the situation under some control, limits obtained in [186] were presented only when (in simulated events) $Q<m_{V}$, for $\sqrt{g_{f} g_{\chi}}=1$, or $4 \pi$ (i.e. when the perturbative approach was still valid). This procedure was referred to as truncation. For the effective operators D5 (vector), D8 (axial vector), and D9 (tensor) from Table 1 these truncated limits are collected in Fig. 7, 8, and 9 of [186].

Finally these $M_{*}$-limits were translated, according to [145], into exclusion curves for the WIMP-nucleon interaction cross section as a function of $m_{\chi}$. The results are shown in Fig. 33 for spin-independent (D5) and spin-dependent (D8, D9) $\chi$-nucleon interactions and are compared to other measurements from various DM search experiments.

One can conclude from the figure that the LHC search for WIMP pair production accompanied by an energetic $\gamma$-quanta traditionally extends the limits on the $\chi$-nucleon scattering cross section to the low-mass region of $m_{\chi}<10 \mathrm{GeV} / c^{2}$. In fact, it brings nothing new.

The $\gamma+E_{\mathrm{T}}^{\text {miss }}$ data were used in [186] to constrain another DM model, which coupled directly WIMPs and SM gauge bosons [187]. The effective WIMP couplings to different bosons were parametrized by the coupling strengths $k_{1}$ and $k_{2}$, which correspond to the $\mathrm{U}(1)$ and $\mathrm{SU}(2)$ gauge sectors of the SM. WIMP production in the model via $p p \rightarrow \gamma+X \rightarrow \gamma \chi \bar{\chi}+X^{\prime}$, does not require any initial-state radiation (Fig. 34). This model can also describe the line near 130 $\mathrm{GeV}$ in the Fermi-LAT $\gamma$-ray spectrum [188, and allows a direct comparison of the Fermi-LAT and the ATLAS data in the same parameter space.

For this model limits were placed on the effective mass scale $M_{*}$ in the $\left(k_{2}, k_{1}\right)$ parameter plane, as shown in Fig. 35. The exclusion line is drawn by considering the value of $M_{*}$ needed to generate the $\chi \bar{\chi} \rightarrow \gamma \gamma$ annihilation rate consistent with the observed Fermi-LAT $\gamma$-ray line. 


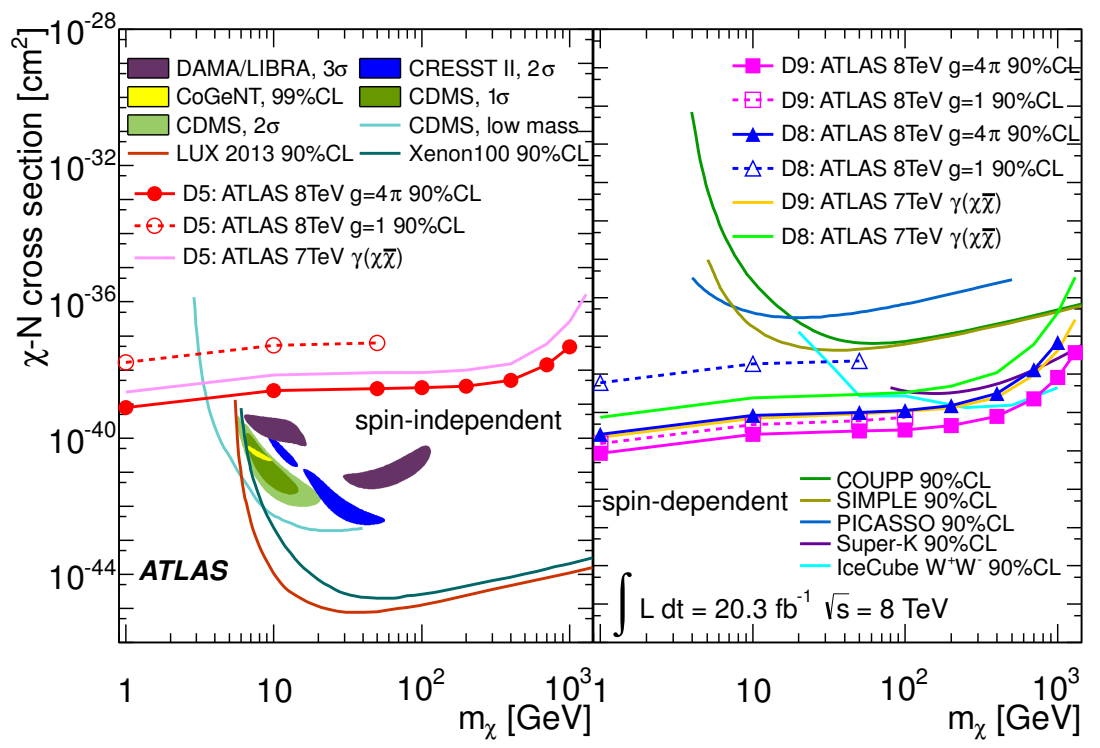

Figure 33. Upper limits $(90 \% \mathrm{CL})$ on the $\chi$-nucleon cross section as a function of $m_{\chi}$ for spin-independent (left) and spin-dependent (right) interactions. The truncation procedure is applied and coupling strength $g \equiv \sqrt{g_{f} g_{\chi}}=1$, or $4 \pi$. The previous ATLAS results obtained with $7 \mathrm{TeV}$ data and results from DM search experiments [70, 180, 84, 87, 91, 73, 88, 172, 173, 79, 81, 85, 42, are also shown. From [186].

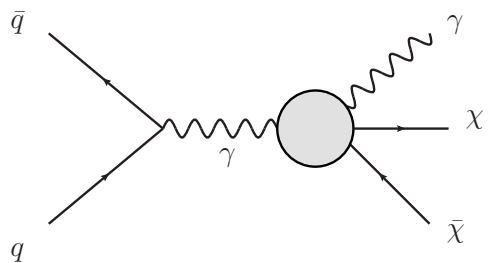

Figure 34. Production ( $s$-channel) of pairs of WIMP particles $(\chi \bar{\chi})$ via an effective $\gamma \gamma \chi \bar{\chi}$ vertex. From [186].

Contrary to the EFT problem with verification of validity (by means of truncation), any simplified model with explicit mediator is ultraviolet complete and therefore robust for all values of $Q$. For such a simplified $Z^{\prime}$-like model with vector interactions and mediator width $\Gamma=m_{V} / 3$, Fig. 14 from [186] shows the limits on the coupling parameter $\sqrt{g_{f} g_{\chi}}$ calculated for various values of the WIMP and mediator particle masses, and compared to the lower limit resulting from the relic DM abundance [189]. This competition between the relic DM abundance limits and LHCfound limits looks very complicated and not very impressive.

Furthermore, for this model with $Z^{\prime}$-like mediator [146] limits on $M_{*}$ as a function of $m_{V}$ are shown for vector (Fig. 36) and for axial-vector (Fig. 37) interactions. The limits are given for two representative WIMP masses $m_{\chi}$ of 50 and $400 \mathrm{GeV} / c^{2}$. The $M_{*}-m_{V}$ contours (thin lines) for an overall coupling $\sqrt{g_{f} g_{\chi}}=0.1,0.2,0.5,1,2,5,4 \pi$ are also shown in both figures.

One can see that when $m_{V}$ is greater than the LHC reach, the EFT approach provides a good approximation of the simplified model. The EFT limits look always more conservative than those from the simplified model as long as $m_{V}$ is greater than or equal to the value used for 


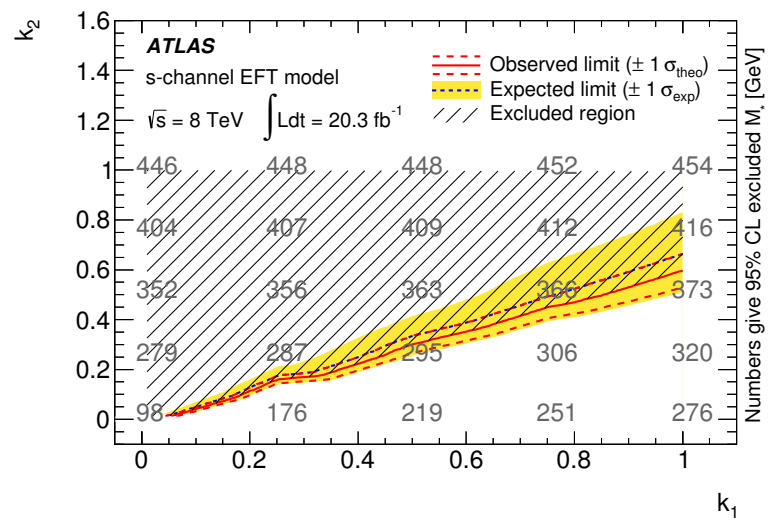

Figure 35. Limits at $95 \% \mathrm{CL}$ on the effective mass scale $M_{*}$ in the $\left(k_{2}, k_{1}\right)$ parameter plane for the $s$-channel EFT model inspired by Fermi-LAT $\gamma$-ray line, for $m_{\chi}=130$ $\mathrm{GeV} / c^{2}$. The upper part of the plane is excluded. From [186].

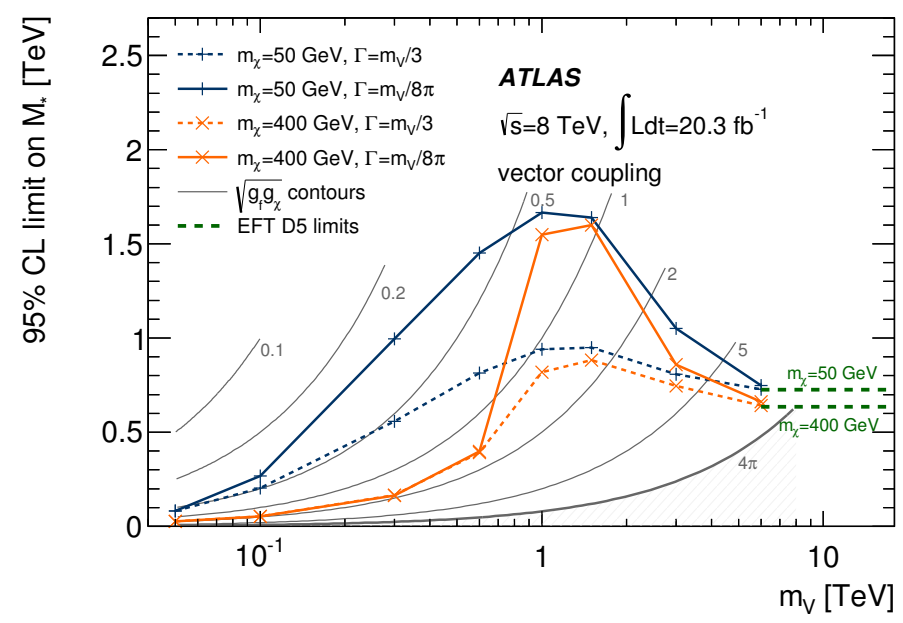

Figure 36. Observed lower limits at 95\% CL on the EFT suppression scale $M_{*}$ as a function of the mediator mass $m_{V}$, for a $Z^{\prime}$-like mediator with vector interactions. Results are shown for different values of the mediator total decay width $\Gamma$ and compared to the EFT observed limit results for a D5 (vector) interaction (dashed line). From [186].

EFT truncation. This can be seen by comparing the $M_{*}$ limits derived from the EFT approach using truncation to those of the simplified model, recalling that $m_{V}=M_{*} \sqrt{g_{f} g_{\chi}}$.

Finally observed $\gamma+E_{\mathrm{T}}^{\text {miss }}$ distributions were used in [186 for supersymmetry constraints. Collisions of protons could result in pair production of squarks, $\tilde{q}$, which could decay to a SM quark and a stable neutralino $\tilde{\chi}_{1}^{0}$. If the mass difference $m_{\tilde{q}}-m_{\tilde{\chi}_{1}^{0}}$ is small, the SM quarks would have very low momentum and would therefore not be reconstructed as jets. Again, the radiation of a photon either from an initial-state quark or an intermediate squark would result in $\gamma+E_{\mathrm{T}}^{\text {miss }}$ events, as shown in Fig. 38 .

One can conclude that the data of [186] are well described by the expected SM backgrounds. The observed upper limit on the fiducial cross section for the production of $\gamma+E_{\mathrm{T}}^{\text {miss }}$ events is $5.3 \mathrm{fb}(95 \% \mathrm{CL})$. More sophisticated analysis was given, validity of the EFT was discussed. 


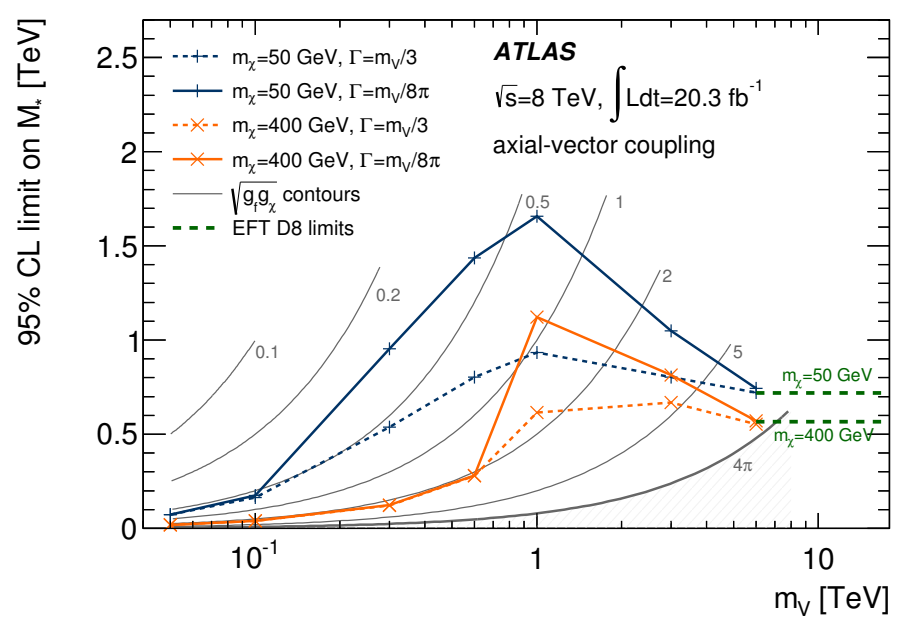

Figure 37. Observed limits at 95\% CL on the EFT suppression scale $M_{*}$ as a function of the mediator mass $m_{V}$, for a $Z^{\prime}$-like mediator with axial-vector interactions. Results are shown for different values of the mediator total decay width $\Gamma$ and compared to the EFT observed limit results for a D8 (axial-vector) interaction (dashed line). From [186].

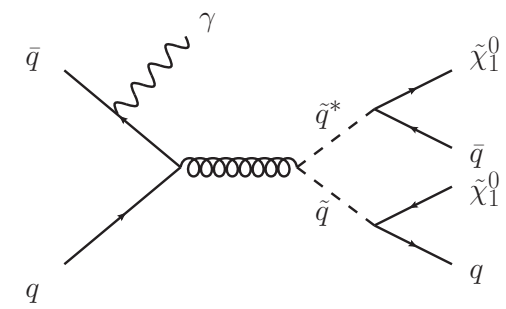

Figure 38. Pair production of squarks $(\tilde{q})$, followed by decay into quarks and neutrali$\operatorname{nos}\left(\tilde{\chi}_{1}^{0}\right)$. From [186].

Exclusion limits were presented on models of new phenomena with large extra spatial dimensions, supersymmetric quarks, and direct pair production of WIMP DM candidates [186]. Nevertheless, the paper leaves a feeling that one sinks into a set of quasi-model-independent receipts with a number of parameters of low physical meaning.

Continuation of the Search for new particles in events with one lepton and missing transverse momentum in pp collisions at $\sqrt{s}=8 \mathrm{TeV}$ with the ATLAS detector using $20.3 \mathrm{fb}^{-1}$ of collected data was published in [190]. For measured electrons and muons Fig. 39 shows the $p_{\mathrm{T}}, E_{\mathrm{T}}^{\mathrm{miss}}$, and $m_{\mathrm{T}}$ spectra after final event selection. The expected SM background and three examples of $W^{\prime}$-boson signals at different masses are also given in the Figure.

The transverse mass variable $m_{\mathrm{T}}=\sqrt{2 p_{\mathrm{T}} E_{\mathrm{T}}^{\text {miss }}\left(1-\cos \varphi_{\ell \nu}\right)}$ was used to identify the signal. Here $p_{\mathrm{T}}$ is the lepton transverse momentum, $E_{\mathrm{T}}^{\text {miss }}$ is the magnitude of the missing transverse momentum vector and $\varphi_{\ell \nu}$ is the angle between the $p_{\mathrm{T}}$ and $E_{\mathrm{T}}^{\text {miss }}$ vectors. The $E_{\mathrm{T}}^{\text {miss }}$ in each event is evaluated by summing over energy-calibrated physics objects (jets, photons and leptons) and adding corrections for calorimeter deposits not associated with these objects [190].

One can see agreement between the data and the predicted SM background for events with $m_{\mathrm{T}}<252 \mathrm{GeV}$, the lowest $m_{\mathrm{T}}$ threshold used to search for a new physics. The value of the 

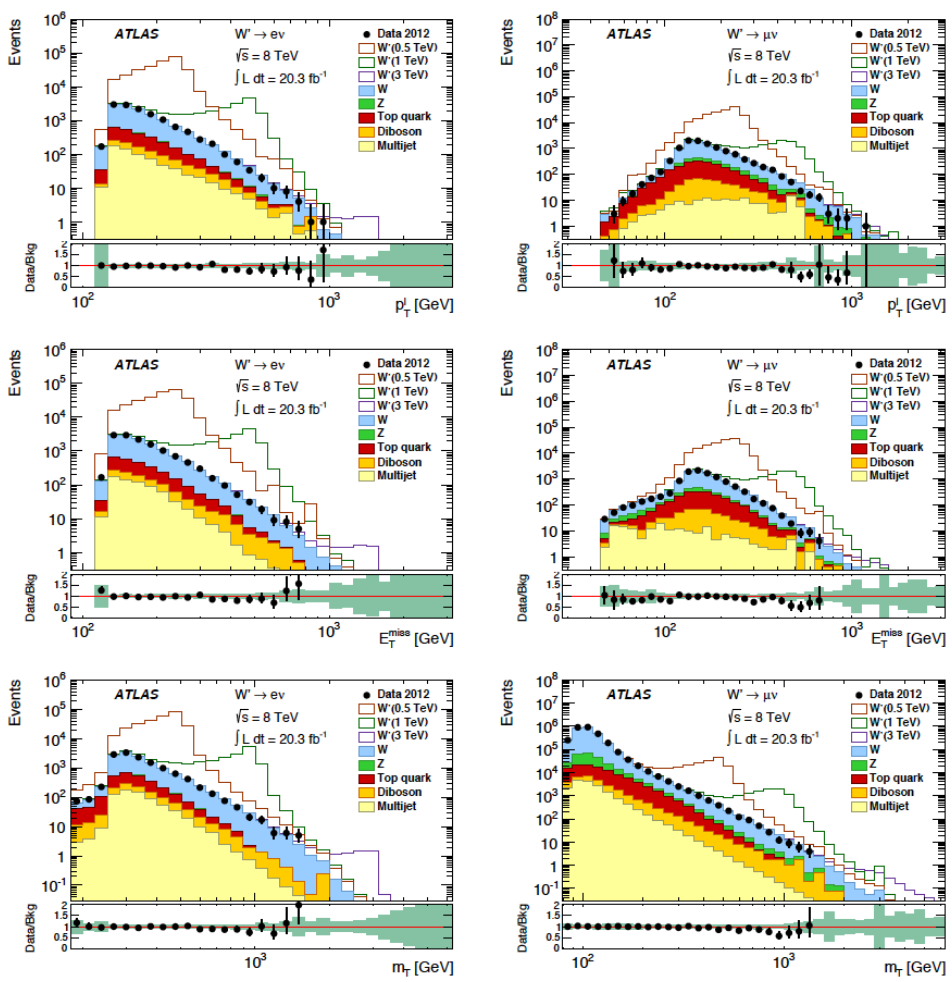

Figure 39. Spectra of lepton $p_{\mathrm{T}}$ (top), $E_{\mathrm{T}}^{\text {miss }}$ (centre) and $m_{\mathrm{T}}$ (bottom) for the electron (left) and muon (right) channels. The spectra of $p_{\mathrm{T}}$ and $E_{\mathrm{T}}^{\text {miss }}$ are shown with the requirement $m_{\mathrm{T}}>252 \mathrm{GeV}$. The ratio of the data to the total background prediction is shown below each of the distributions. From [190.

$m_{\mathrm{T}}$ threshold was a result of an optimization procedure. No significant excess beyond SM expectations is observed.

The mono-lepton data spectra (Fig. 39) were used to constrain direct production of WIMP DM candidates, which expected to be pair-produced, $p p \rightarrow \chi \bar{\chi}+X$, via some non-SM intermediate state. To obtain these constraints the EFT contact operators D1 (scalar), D5c (vector, with constructive interference) and D5d (vector, with destructive interference) and D9 (tensor) from Table 1 were used.

On this basis the new results of the ATLAS search for pair production of WIMP particles in association with a leptonically decaying $W$-boson at $8 \mathrm{TeV}$ (Fig. 39 ) were transformed into limits on $M_{*}$ (Fig. 40) and into exclusion curves for the WIMP-nucleon scattering cross-section (Fig. 41). Both are shown as a function of the WIMP mass $m_{\chi}$. Results of the previous ATLAS searches for $W / Z$-boson decaying hadronically [169], $Z$-boson decaying leptonically [181], and $j+\chi \chi[151]$ are also given in the figures.

One can see that the WIMP production signature with hadronical $W$ decays gave a factor of 1.5 stronger limits on $M_{*}$ with respect to the signature with leptonical $W$ decays. The limits in Fig. 40 were expected to be stable down to arbitrarily small values. One should note that the comparison between direct detection and ATLAS results (given in Fig. 41) is only possible within the validity of the EFT formalism (section 8) which was not discussed in [190].

Besides looking for the WIMP pair production some other Beyond-SM results were obtained in [190]. In particular, a $W^{\prime}$-boson with Sequential SM couplings was excluded for masses up to 


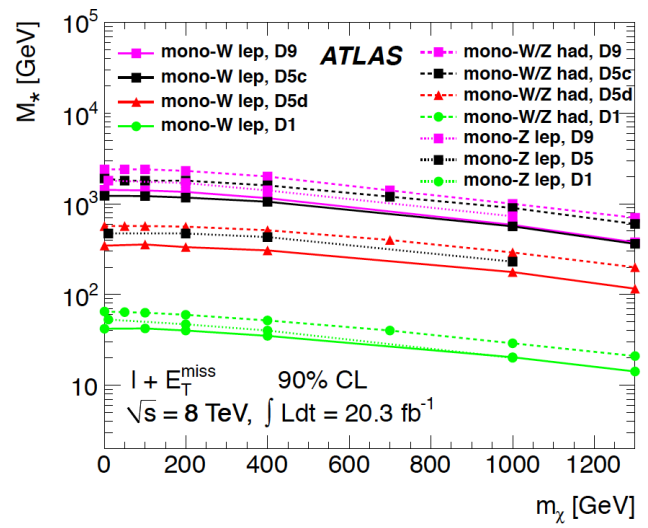

Figure 40. Observed limits for various EFT operators on $M_{*}$ as a function of the $m_{\chi}$ at 90\% CL for the combination of the $e$ - and $\mu$-channel. The values below the corresponding line are excluded. Results of the previous ATLAS searches are also shown. From [190].

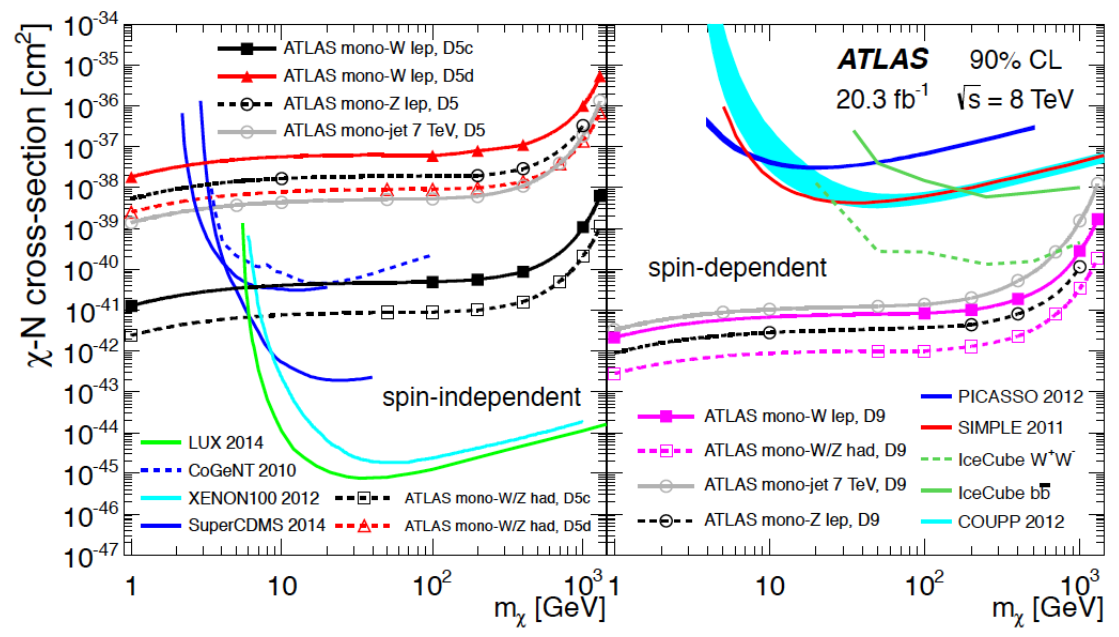

FIgURE 41. Observed limits on the WIMP-nucleon scattering cross-section as a function of $m_{\chi}$ at $90 \%$ CL for spin-independent (left) and spin-dependent (right) EFT operators. Results are compared with the previous ATLAS searches and with direct detection searches by CoGeNT [157, XENON100 [71, CDMS [180, 82, LUX [73, COUPP [173], SIMPLE [91], PICASSO [87] and IceCube [172. From [190].

$3.24 \mathrm{TeV} / c^{2}$, and excited chiral $W^{*}$-bosons [191, 192] with equivalent coupling strengths were excluded for masses up to $3.21 \mathrm{TeV} / c^{2}$.

Much more sophisticated analysis of the mono-jet signature was carried out with $20.3 \mathrm{fb}^{-1}$ of data in the paper [153] titled as Search for new phenomena in final states with an energetic jet and large missing transverse momentum in pp collisions at $\sqrt{s}=8$ TeV with the ATLAS detector.

Remember that the signature with an energetic jet and large $E_{\mathrm{T}}^{\mathrm{miss}}$ is considered as a very distinctive tool for a new physics search at colliders. These "monojet-like" (mono- $\gamma$, mono- $W / Z$, mono- $H$, etc) final states were already studied [193, 194, 168, 195, 167, 196, 151, 166, 197, 183, 181, 169, 198, in the searches for SUSY, large extra dimensions, and WIMPs as candidates for DM. 
The mono-jet events for the study were required in [153] to have no leptons at all and at least one jet with $p_{\mathrm{T}}>120 \mathrm{GeV} / c$. The missing transverse momentum of these events were varied between $E_{\mathrm{T}}^{\text {miss }}>150 \mathrm{GeV}$ and $E_{\mathrm{T}}^{\text {miss }}>700 \mathrm{GeV}$. The full data selected and the expected SM background are presented in Tables 4 and 5 of original paper [153].

Several measured distributions for three signal regions SR1 $\left(E_{\mathrm{T}}^{\text {miss }}>150 \mathrm{GeV}\right), \operatorname{SR} 7\left(E_{\mathrm{T}}^{\mathrm{miss}}>500\right.$ $\mathrm{GeV})$ and $\mathrm{SR} 9\left(E_{\mathrm{T}}^{\mathrm{miss}}>700 \mathrm{GeV}\right)$ together with the SM expectations are shown in Fig. 42 and Fig. 43. For illustration purposes, the figures include the impact of different Beyond-SM (ADD,

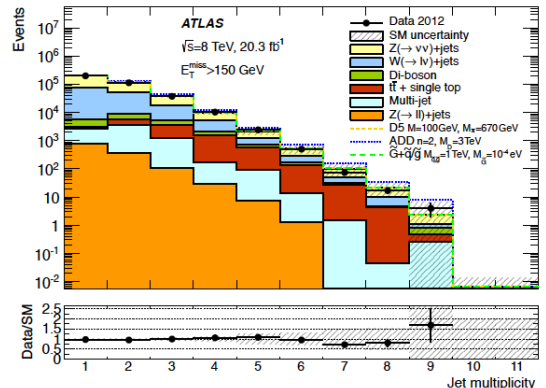

(a)

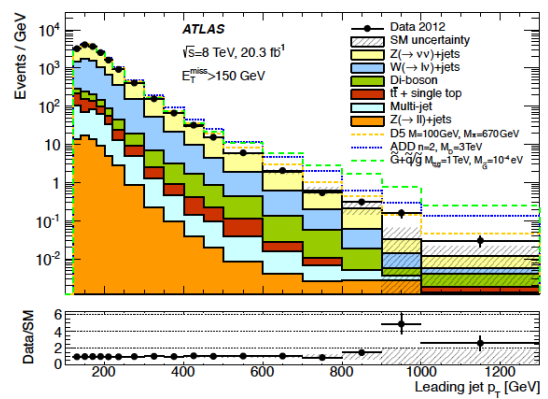

(c)

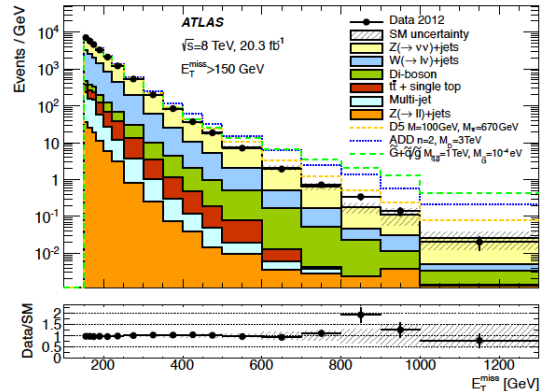

(b)

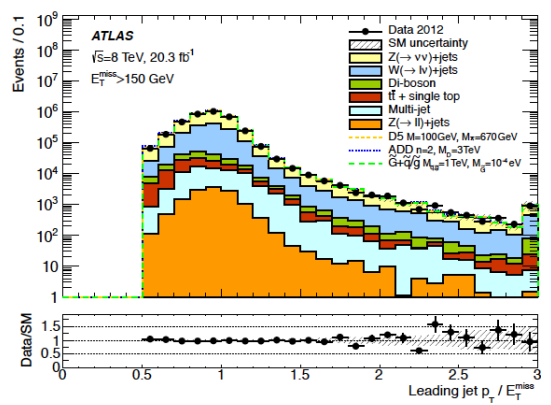

(d)

Figure 42. Measured distributions of (a) the jet multiplicity, (b) $E_{\mathrm{T}}^{\mathrm{miss}}$, (c) leading jet $p_{\mathrm{T}}$, and $(\mathrm{d})$ the leading jet $p_{\mathrm{T}}$ to $E_{\mathrm{T}}^{\text {miss }}$ ratio for the $\mathrm{SR} 1$ selection compared to the $\mathrm{SM}$ expectations. From 153.

WIMP, and GMSB SUSY) scenarios.

In general, good agreement was observed between the data and the SM expectations. The largest difference ( $1.7 \sigma$ deviation) between the number of events in the data and the expectations was observed for $E_{\mathrm{T}}^{\text {miss }}>700 \mathrm{GeV}$ (signal region SR9).

The agreement between the data and the SM expectations was used to put model-independent upper limits (Table 3) on the visible BSM cross section $\sigma \times A \times \epsilon$ (cross section $\times$ acceptance $\times$ efficiency), using approach of [199] and the systematic uncertainties on the backgrounds and the uncertainty on the integrated luminosity. The Table shows that visible cross sections $\sigma \times A \times \epsilon$ above $599 \mathrm{fb}-2.9 \mathrm{fb}$ are excluded at $90 \%$ CL for SR1-SR9 selections, respectively. Simulation of background processes $Z(\rightarrow \nu \bar{\nu})+$ jets allowed one to find that typical event selection efficiencies $\epsilon$ vary from $88 \%$ for SR 1 and $83 \%$ for SR3 to $82 \%$ for SR7 and $81 \%$ for SR9.

The main model-independent results of [153] given in Table 3 and in Fig. 42 and Fig. 43 can be transformed into exclusion limits on pair production of WIMP DM candidates, and further, for example, on models with large extra spatial dimensions and production of very light gravitinos in a gauge-mediated SUSY model. 

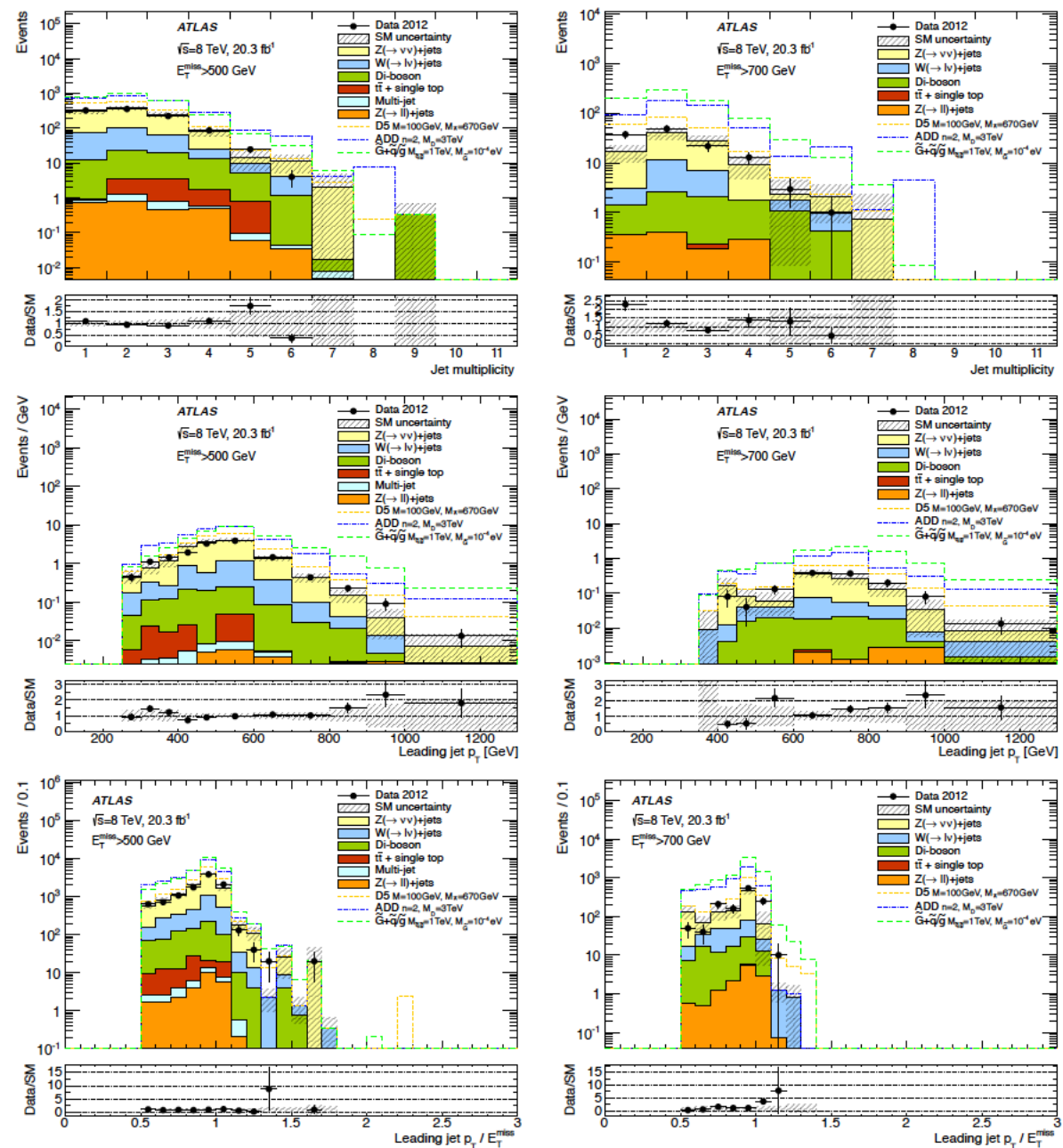

(a)

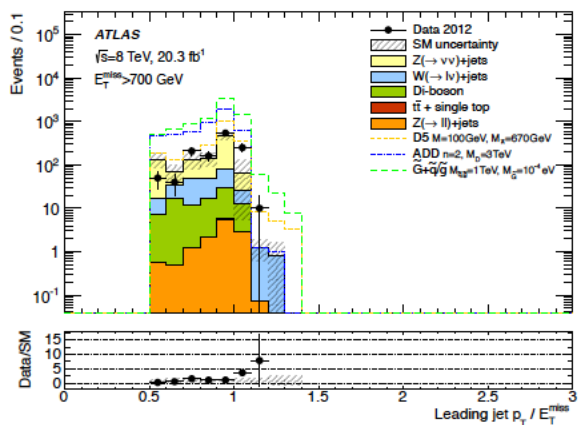

(b)

FiguRE 43. Measured distributions of the jet multiplicity, leading jet $p_{\mathrm{T}}$, and the leading jet $p_{\mathrm{T}}$ to $E_{\mathrm{T}}^{\text {miss }}$ ratio for (a) SR7 and (b) SR9 selections compared to the SM expectations. From [153].

TABLE 3. Observed (expected) $90 \%$ and $95 \%$ CL upper limits on the visible cross section $\sigma \times A \times \epsilon$ in fb for the SR1-SR9 selections. From [153].

\begin{tabular}{|l|c|c|}
\hline Signal Region & $90 \%$ CL & 95\% CL \\
\hline $\mathrm{SR} 1, E_{\mathrm{T}}^{\text {miss }}>150 \mathrm{GeV}$ & $599(788)$ & $726(935)$ \\
$\mathrm{SR} 2, E_{\mathrm{T}}^{\text {miss }}>200 \mathrm{GeV}$ & $158(229)$ & $194(271)$ \\
$\mathrm{SR} 3, E_{\mathrm{T}}^{\text {miss }}>250 \mathrm{GeV}$ & $74(89)$ & $90(106)$ \\
$\mathrm{SR} 4, E_{\mathrm{T}}^{\text {miss }}>300 \mathrm{GeV}$ & $38(43)$ & $45(51)$ \\
$\mathrm{SR} 5, E_{\mathrm{T}}^{\text {miss }}>350 \mathrm{GeV}$ & $17(24)$ & $21(29)$ \\
$\mathrm{SR} 6, E_{\mathrm{T}}^{\text {miss }}>400 \mathrm{GeV}$ & $10(14)$ & $12(17)$ \\
$\mathrm{SR} 7, E_{\mathrm{T}}^{\text {miss }}>500 \mathrm{GeV}$ & $6.0(6.0)$ & $7.2(7.2)$ \\
$\mathrm{SR} 8, E_{\mathrm{T}}^{\text {miss }}>600 \mathrm{GeV}$ & $3.2(3.0)$ & $3.8(3.6)$ \\
$\mathrm{SR} 9, E_{\mathrm{T}}^{\text {miss }}>700 \mathrm{GeV}$ & $2.9(1.5)$ & $3.4(1.8)$ \\
\hline
\end{tabular}


As already discussed above, any search for WIMPs at a collider, in particular at the LHC [200], is an important possibility of spreading some light on the DM problem. To fit the correct relic density for non-relativistic cold DM in the early universe [18] the WIMP masses are allowed to be between a few $\mathrm{GeV} / c^{2}$ and a $\mathrm{TeV} / c^{2}$, and they are expected to interact, despite gravity, only (very) weakly. Like ordinary neutrinos, WIMPs will escape detection because they ca not deposit any measurable amount of energy in a calorimeter. Therefore, one inevitably concludes that WIMP production at a collider can be noticed only by means of large transverse momentum imbalance $\left(p_{\mathrm{T}}^{\mathrm{miss}}\right)$ of ordinary particles, the magnitude of which is called $E_{\mathrm{T}}^{\mathrm{miss}}$.

To convert the main model-independent results of [153] into numerical constraints on the DM problem, one traditionally used the EFT approach, where the effective contact operators (Table 1) describe WIMP-SM interaction, which in fact could be mediated by a single new heavy particle or particles with mass too large to be produced directly at the LHC (Fig. 44(left)).
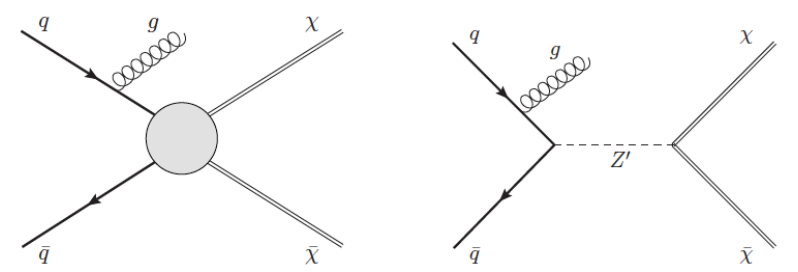

Figure 44. Feynman graphs for production of WIMP pairs $\chi \bar{\chi}$ associated with a jet from initial-state radiation of a gluon, $g$, via a contact effective operator (left), and in a simplified model with a $Z^{\prime}$ intermediate boson (right). From [153].

The representative set of seven operators (Table 1) included C1 (scalar), D1 (scalar), D5 (vector), D8 (axial-vector), D9 (tensor), C5 (scalar), D11(scalar) operators. The first five describe bilinear quark couplings to WIMPs, $q \bar{q} \rightarrow \chi \bar{\chi}$, the latter two describe $g g \rightarrow \chi \bar{\chi}$ couplings. Despite the serious questions about the validity of the EFT approach (section 8), one is forced to use it due to a lack of a reasonable alternative approach for comparing LHC results with results of direct and indirect DM searches.

From signal regions exhibiting the best expected sensitivity, for each EFT operator under consideration one first extracted the limits on $M_{*}$ as a function of $m_{\chi}$ (Fig. 45). The $1 \sigma$ and $2 \sigma$ error bands around the expected limit are due to the acceptance uncertainties. In particular, the main sources of experimental uncertainties were the parton-shower matching scale (5\%), jet and $E_{\mathrm{T}}^{\text {miss }}$ energy scale (up to $10 \%$ ), and PDF (5-29\%). The main sources of theoretical uncertainties were variation of the renormalization and factorization scales of the EFT operators (up to $46 \%$ ), and uncertainty due to the PDF for the operators (20-70\%). The effect of the beam-energy uncertainty $(2-9 \%)$ on the observed limit was negligible [153].

Looking at Fig. 45 one agrees with [153] that a demonstration of the EFT validity could be done by relating the scale $M_{*}$ to the mass of a mediator $m_{V}$ and the coupling constants $g_{i}$ by $m_{V}=f\left(g_{i}, M_{*}\right)$. The explicit form of the function $f$ depends on the concrete operator. For a given operator, the validity criterion is $Q<m_{V}$, where $Q$ is a momentum transferred in the hard interaction. Following this criterion, events were excluded from the analysis and omitted in Fig. 45. The criterion also depends on $g_{i}$, for which one considers two possibilities: $g_{i}=1$, and the maximum possible coupling to stay in the perturbative regime $\left(\sqrt{g_{i} g_{j}}=4 \pi\right)$. 


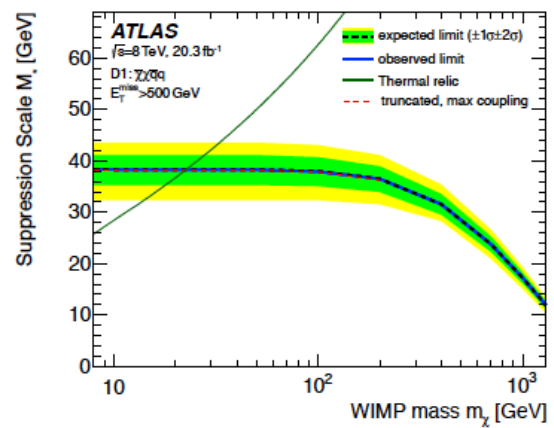

(a)

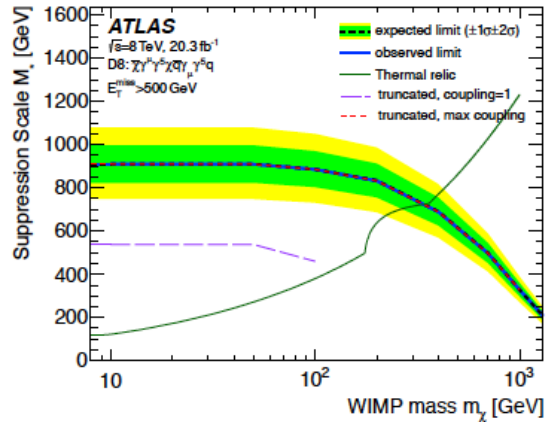

(c)

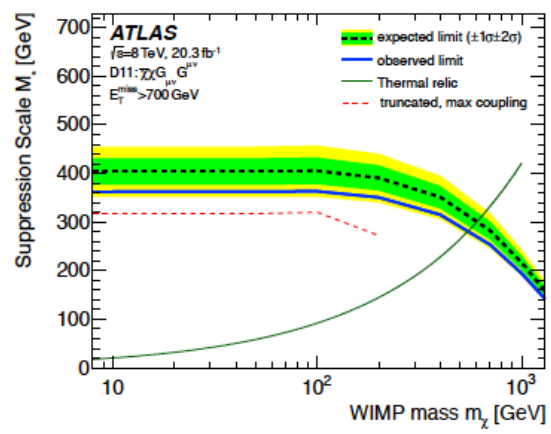

(e)

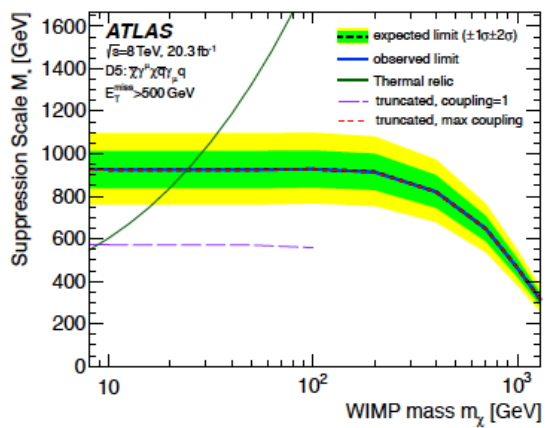

(b)

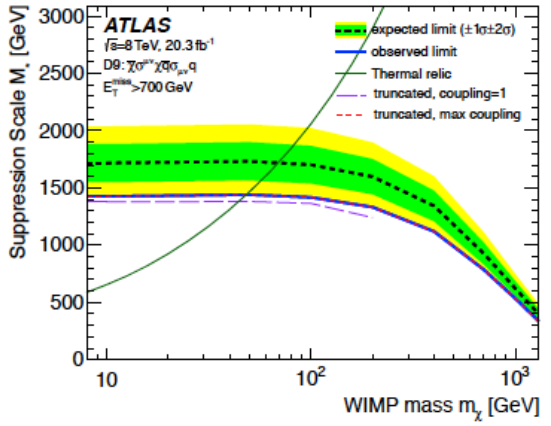

(d)

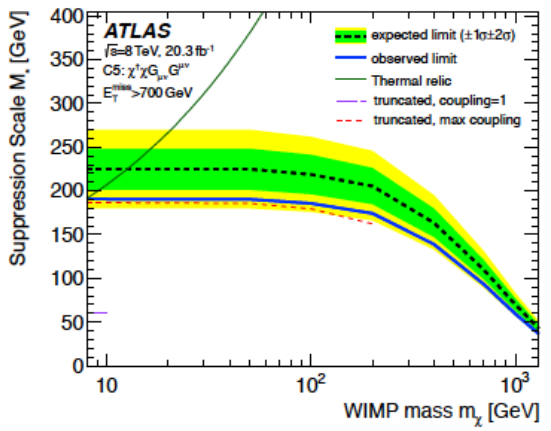

(f)

Figure 45 . Lower limits at $95 \% \mathrm{CL}$ on the scale $M_{*}$ are shown as a function of the WIMP mass $m_{\chi}$ for (a) D1, (b) D5, (c) D8, (d) D9, (e) D11 and (f) C5 operators. The expected and observed limits are shown as dashed black and solid blue lines, respectively. The rising green lines are the $M_{*}$ values at which WIMPs of the given mass result in the relic density as measured by WMAP [201, assuming annihilation in the early universe proceeded exclusively via the given operator. The purple long-dashed line is the $95 \% \mathrm{CL}$ observed limit on $M_{*}$ imposing a validity criterion with a coupling strength of 1 , the red dashed thin lines are those for the maximum physical coupling strength. From [153].

After reducing the signal cross section according to the criterion, the scale $M_{*}$ was recalculated in two expected truncated limit lines in Fig. 45. The truncated limits fulfill the validity criteria wherever the lines are drawn in the figure.

Finally, using D9 as an example, one can see that the maximum coupling criterion is fulfilled for all WIMP masses, the $g_{i}=1$ criterion is fulfilled for $m_{\chi}<200 \mathrm{GeV} / c^{2}$. For C5, the validity criterion for $g_{i}=1$ is violated over almost the whole WIMP mass range, and a truncated limit line is only drawn up to a WIMP mass of $10 \mathrm{GeV} / c^{2}$. 
For completeness, Fig. 45 also includes WIMP thermal relic curves, calculated for only onein-time effective operator in question [145, with the WIMP-SM coupling, that for fixed $M_{*}$ and $m_{\chi}$ gives the correct relic abundance. Remarkably, the D8-thermal-relic line has a bump at the top-quark mass where the annihilation channel to top quarks opens [153]. Under the assumption that true DM is entirely composed of these thermal relics, the limits on $M_{*}$ which are above the value required for the thermal relic density exclude the case where WIMPs annihilate to SM particles via the corresponding operator.

This discussion, taken almost completely from [153], demonstrates to an unbiased reader how complicated, poor controlled, and, therefore, rather useless are all these attempts to stay within the EFT approach. Furthermore, one can notice that the variation of upper limits on the scale $M_{*}$ as a function of an effective operator looks very substantial. Several auxiliary curves (with truncated limits and for relic abundance) in the figures convince the reader in the high accuracy level of the analysis, but make the impression of the figures very complicated and do not clarify the usefulness of these curves.

To obtain quantitative limits on WIMP pair production a so-called simplified model was used in addition to the EFT approach in [153] for alternative description of the WIMP-pair production. In the model the WIMPs couple to a $q$-pair explicitly via a new vector $Z^{\prime}$ boson (Fig. 44(right)) with mass $m_{V}$ and width $\Gamma$. In this case only coupling of $q \bar{q} \rightarrow \chi \bar{\chi}$ can be probed and the product of the coupling constants $\sqrt{g_{q} g_{\chi}}$ can be constrained. Since in the model one explicitly has $M_{*}=m_{V} / \sqrt{g_{q} g_{\chi}}$, this equality can be given as a point in the $M_{*}-m_{V}$ plane in Fig. 46(a). Therefore for a given $m_{V}$ and two representative values of $\Gamma$, one can compare
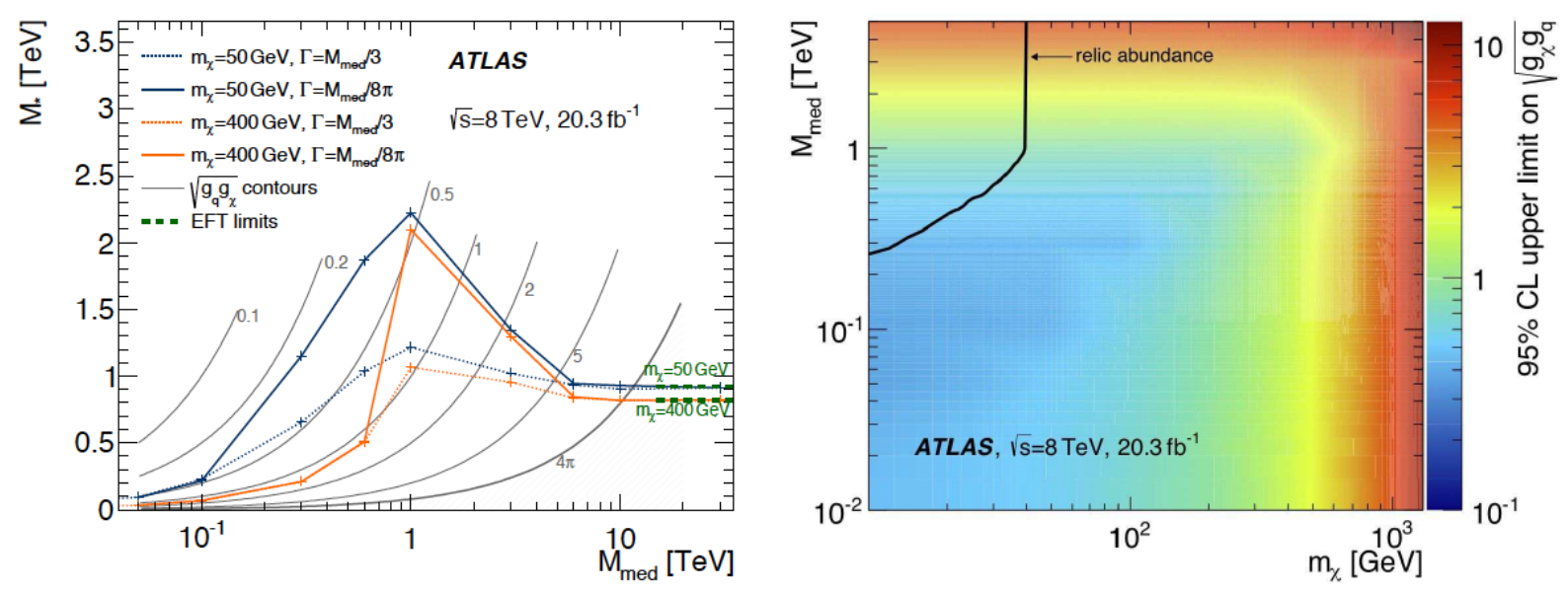

Figure 46. (a) Observed 95\% CL limits on the suppression scale $M_{*}$ as a function of the mediator mass $M_{\text {med }} \equiv m_{V}$, for $m_{\chi}=50$ and $400 \mathrm{GeV} / c^{2}$. The width of the mediator $\Gamma=m_{V} / 3$, or $m_{V} / 8 \pi$. The corresponding limits from EFT models are shown as dashed lines; contour lines indicating a range of values of $\sqrt{g_{q} g_{\chi}}$ are also shown. (b) Observed 95\% CL upper limits on $\sqrt{g_{q} g_{\chi}}$ in the plane of $M_{\text {med }}$ versus $m_{\chi}$. Values leading to the correct relic abundance [201] are shown by the black solid line. From [153].

the "true" above-mentioned value of the $M_{*}$ with the $M_{*}$ value (shown as dashed line) derived assuming a contact interaction.

From the figure one confirms the rather obvious point that the contact interaction can work only for $m_{V} \geq 5 \mathrm{TeV} / c^{2}$ because in the intermediate range $\left(700 \mathrm{GeV} / c^{2}<m_{V}<5 \mathrm{TeV} / c^{2}\right)$ the 
mediator can be produced resonantly and the true $M_{*}$ is higher than the $M_{*}$ obtained in the contact interaction regime. In this case the contact interaction limits will be pessimistic. Next, smaller mediator masses $m_{V}<700 \mathrm{GeV} / c^{2}$ give smaller true $M_{*}$ limits because once $m_{\chi}>m_{V}$ the WIMP pair production via this mediator will be kinematically suppressed. In this region, the contact interaction limits would be optimistic and overestimate the true $M_{*}$ values [153]. In this situation a question survives: How could one know which mediator mass "works today"?

In Fig. 46(b) the observed upper limits on the product of couplings of the simplified model vertex $\sqrt{g_{q} g_{\chi}}$ are shown in the $m_{V}-m_{\chi}$ plane. Within this model, the regions to the left of the correct-relic-density line lead to the values of the relic density larger than measured and are excluded.

Therefore the use of a simplified model allows one to avoid the poorly controlled problems of the EFT validity, to obtain some feeling about the EFT validity, but as a price an extra parameter dependence appears and the obtained constraints become more and more complicated.

Unfortunately, at the moment the EFT approach looks inevitable if one wants to obtain from Fig. 45 some valuable constraints on possible WIMP-nucleon SD and SI couplings. Therefore, as before, for example in [151], these $M_{*}-m_{\chi}$ limits with relevant effective operators were converted into exclusion curves for the SD and SI WIMP-nucleon scattering cross sections.

The ATLAS-obtained exclusion curves (Fig. 47) look particularly relevant for low WIMP masses and remain important for all $m_{\chi}$. The spin-dependent ATLAS exclusion curves in Fig. 47(b) are based on limits from the D8 (axial-vector) and D9 (tensor) operators. Both of them are significantly stronger than those from direct-detection experiments. The spin-independent ATLAS exclusion curves Fig. 47(a) traditionally look less restrictive.

Figure 47(c) illustrates conversion of the $M_{*}-m_{\chi}$ constraints into upper limits on the WIMP annihilation rate, calculated as the product of annihilation cross section $\sigma$ and the relative WIMP velocity $v$ averaged over the DM velocity distribution $\langle\sigma v\rangle$. Results of vector and axialvector operators describing the annihilations of WIMPs to the four light-quark flavors are only shown. One can compare these limits with those obtained earlier in [151. Limits on the WIMP annihilation to $u \bar{u}$ - and $q \bar{q}$-pairs from galactic high-energy $\gamma$-ray observations by the Fermi-LAT [202] and H.E.S.S. 203] telescopes and the annihilation rate that follows from the thermal relic density measured by WMAP 201] and PLANCK 204] satellites are also shown for comparison.

Figure 47 again concerns the EFT validity problem and shows the effects of the truncation procedure on the upper limits for the considered WIMP observables. In general, the EFT limits remain valid for WIMP masses up to about $200 \mathrm{GeV} / c^{2}$. The effect depends strongly on the operator and the values for the couplings. The allowed variation of the coupling strengths leads to changes in the limits of up to one order of magnitude. Strictly speaking, it is rather difficult to believe in usefulness of such limits.

The mono-jet $+E_{\mathrm{T}}^{\text {miss }}$ experimental data (Figs. 42 and 43 can be used (as before in [174]) for a study of the Higgs boson invisible decays [146]. A sizable value of $\mathrm{BR}(H \rightarrow$ invisible $)$ is not yet experimentally excluded. There are models connecting a "hidden" DM sector with SM particles via direct DM-Higgs-SM-couplings (for example, [205, 206, 207, 177]). In this case the Higgs boson can decay into invisible WIMP DM candidates producing a deviation of a measured SM Higgs branching ratio from the expected one [23]. 


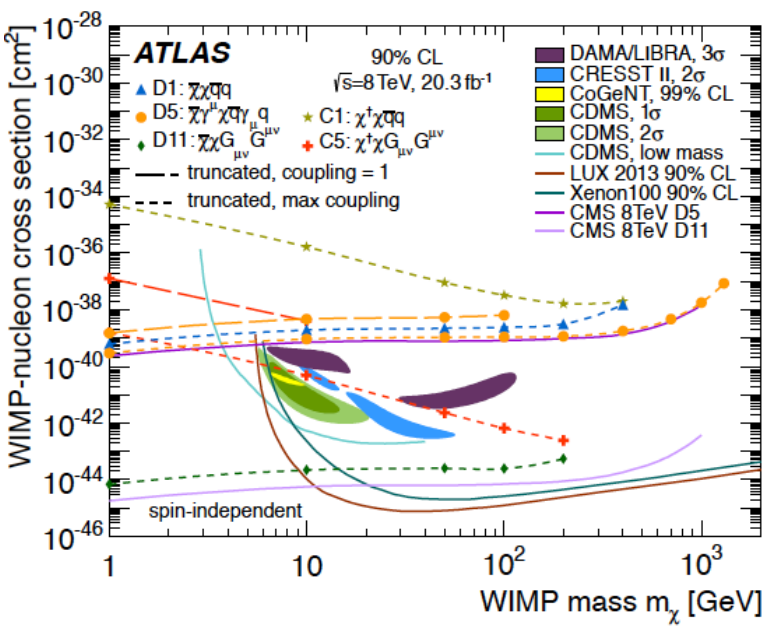

(a)

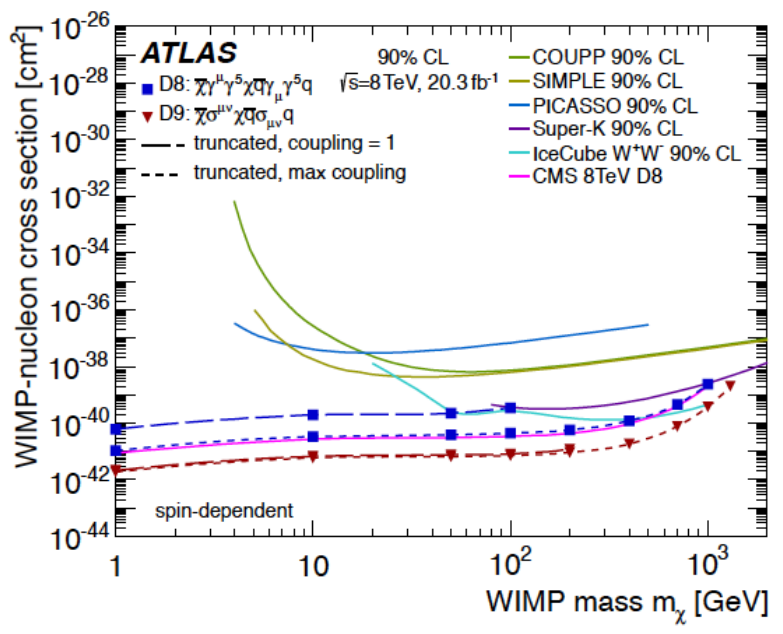

(b)

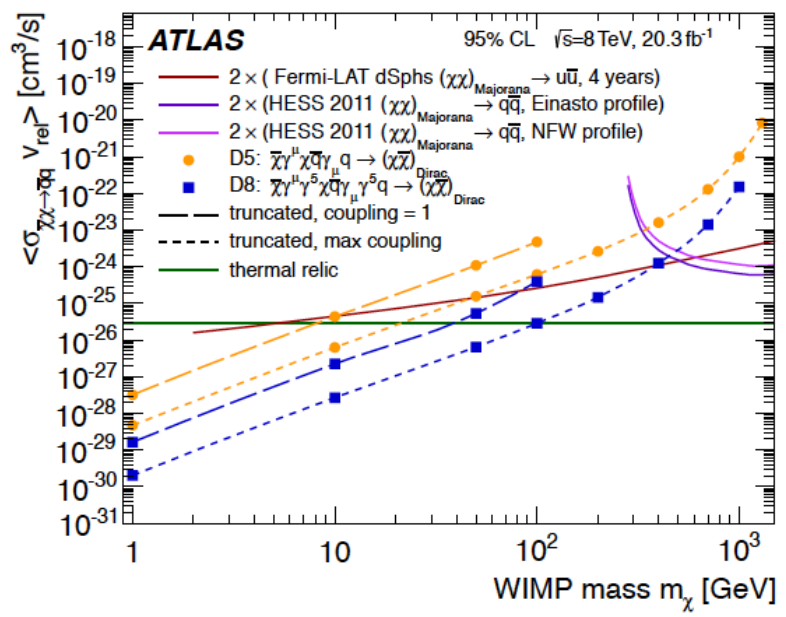

(c)

Figure 47. The exclusion curves for (a) the spin-independent and (b) spin-dependent WIMP-nucleon cross section as a function of the WIMP mass $m_{\chi}$ for different effective operators. Results from direct-detection experiments for the spin-independent [79, 73, 81, 82, 84, 85, 71, and spin-dependent [87, 88, 89, 90, 91, cross section, and the CMS (untruncated) results [197] are shown. (c) The 95\% CL limits on the WIMP annihilation rate as a function of the WIMP mass. Results from $\gamma$-ray telescopes [202, 203] are also shown, along with the thermal relic density annihilation rate [201, 204. From [153].

At the LHC, based on the associate Higgs- $Z$ boson production, the strong upper limits of $58-65 \%$ (at 95\% CL) were already set on the branching ratio for the Higgs invisible decay [174, 208]. This investigation was continued in [153], where the mono-jet $+E_{\mathrm{T}}^{\text {miss }}$ final state was used to search for the production of an invisibly decaying SM Higgs-like boson with an allowed mass range between $115 \mathrm{GeV} / c^{2}$ and $300 \mathrm{GeV} / c^{2}$. Figure 48 shows the observed and expected limits on the production cross section times branching ratio $\sigma \times \mathrm{BR}(H \rightarrow$ invisible $)$ as a function of the boson mass.

Values for $\sigma \times \mathrm{BR}(H \rightarrow$ invisible $)$ above $44 \mathrm{pb}$ for $m_{H}=115 \mathrm{GeV} / c^{2}$ and $10 \mathrm{pb}$ for $m_{H}=$ $300 \mathrm{GeV} / c^{2}$ are excluded. Comparison with previous constraints from the analysis of $Z H(Z \rightarrow$ 


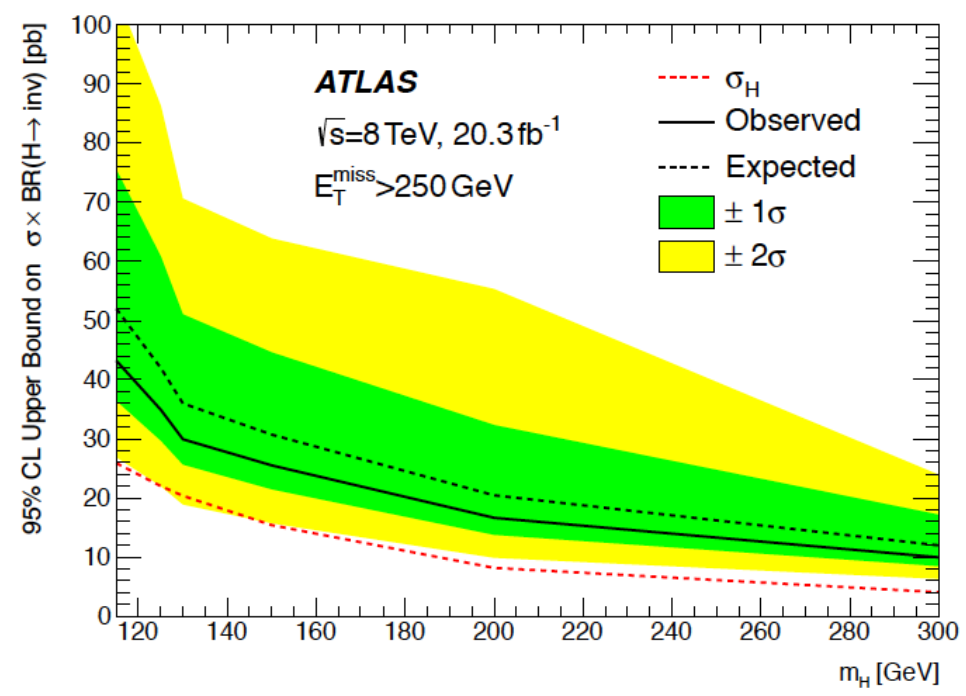

Figure 48. The observed (solid line) and expected (dashed line) 95\% CL upper limit on $\sigma \times \mathrm{BR}(H \rightarrow$ invisible $)$ as a function of the boson mass $m_{H}$. The shaded areas around the expected limit indicate the expected $\pm 1 \sigma$ and $\pm 2 \sigma$ ranges of limits in the absence of a signal. The expectation for a Higgs boson with $\mathrm{BR}(H \rightarrow$ invisible $)=1, \sigma_{H}$, is also shown. From [153].

$\ell^{+} \ell^{-}$) final states [174] shows that the obtained result is less sensitive and does not yet have the sensitivity to probe the SM Higgs boson couplings to invisible particles, at least for Higgs boson mass of $125 \mathrm{GeV} / c^{2}[153$.

Concluding the very sophisticated study of [153] one can say that search for new phenomena in events with an energetic jet and large $E_{\mathrm{T}}^{\text {miss }}$ at $\sqrt{s}=8 \mathrm{TeV}$ did not give evidence for disagreement with the SM expectations. The results were translated into model-independent upper limits on new physics contributions. Furthermore, a very sophisticated analysis of the EFT validity was carried out. To this end a special Appendix was arranged in [153]. The discussion of the subject runs to the higher level.

The latest, to our knowledge, results of the ATLAS search for invisible decays of the Higgs boson produced in association with a hadronically decaying vector boson in $p p$ collisions at $\sqrt{s}$ $=8 \mathrm{TeV}$ are given in [209].

Very similar analyses were carried out by CMS collaboration, and similar results were obtained. In particular, the review Search for Dark Matter at CMS [149] has presented the results from searches for directly produced WIMP particles on the basis of the full LHC RUN I dataset of $20 \mathrm{fb}^{-1}$ at $8 \mathrm{TeV}$. Final states with a mono-jet, mono-photon, and mono-lepton signature were considered, as well as processes with WIMP particles produced in association with top quarks. Most of these results were interpreted using the EFT approach, while results in simplified models were also reported. The latest results of the CMS collaboration DM search are given in [210]. Recent reviews of the subjects can also be found in [23, 150].

In the conclusion of this section (about ATLAS program for the WIMP search at the LHC) one can first point out that unfortunately, despite increasing complexity and increasing accuracy of the analyses performed in 2010-2014, all searches gave no evidence for a signal of physics beyond the SM based on the $E_{\mathrm{T}}^{\text {miss }}$ signature. This is the main physics result of the program. 
Second, the justification of connection of this program with the DM problem relies on a key assumption that the WIMPs looked for at the LHC are equivalent to the true DM particles.

Third, one believes, with all known caveats on the EFT results in mind, a few robust observations can be made on the complementarity between the collider and direct searches for DM particles. The first feature is the strength of the collider analyses searching for low-mass WIMPs with reasonable sensitivity up to zero mass. Nevertheless, a question survives about usefulness of these "zero-mass" results for solution of the DM problem. Nowadays it looks unrealistic that cosmological (cold, warm, etc) DM particle masses could be indeed so small. Obviously, at a rather high WIMP mass, the collider WIMP search potential vanishes due to the drop of the WIMP production cross section [149]. Furthermore, at least today, the direct DM detection technique is more constraining for spin-independent scattering for WIMP masses above a few $\mathrm{GeV} / c^{2}[23]$.

Another complementarity follows from the point that collider people believe that the directdetection experiments have typically reduced sensitivity to spin-dependent interactions, and hence allows the collider searches to constraint severely this kind of interaction at intermediate WIMP masses as well. Nevertheless, it is obvious that this statement has no power to reduce importance of modern direct DM-search experiments especially sensitive to the spin-dependent DM-proton/neutron couplings. The simple reason is that collider constraints concern WIMPs escaping detection, but not true DM particles. This most crucial assumption forces one to be very careful when comparing collider results with direct searches, to say nothing about the specific assumption of EFT or/and many specific variants of a simplified model.

As it pointed, in particular in [146], if WIMP-SM interactions at LHC energies cannot be described by effective operators, the obtained WIMP-SM constraints, depending on the mass and width of the intermediate particle, can become either significantly stronger or considerably weaker. This statement almost kills the meaning of the EFT-based analysis, because today one is unable to know for sure (also a posteriori) which of the unknown intermediate particles really contributed, to say nothing about its mass and width.

Finally, it is reasonable to end this section with the slide (given in Fig. 49 ) from one of a honest discussions of WIMP-DM search at the LHC, which collects the set of assumptions concerning the main subject. It is rather difficult to refrain from a "criminal" thought that here the points are assumed that in fact one should prove experimentally.

- Assuming this particle is cosmologically stable

- Assuming all DM is one type of particle

- Assuming same interaction as for direct detection

- Assuming Effective Field Theory is in range of validity

- Assuming local DM density well known

- Assuming DM velocity distribution is well known

- We find...

FIGURE 49. The main assumptions allowing DM interpretation of the collider WIMP search.

New papers are expected from the LHC on the subject, but it is clear now that they can add very little to solution of the DM problem and one should wait for success of direct DM search experiments. 


\section{Exotic DM SEARCheS}

The lack of clear evidence for Physics beyond SM (BSM), the absence of any SUSY signals and any hints of WIMPs in the first phase of the LHC, insufficient sensitivity of traditional direct and indirect techniques (excluding DAMA/LIBRA) to observe galactic halo DM in a laboratory have all cast some doubts on linking a true DM mass with the electroweak scale [211].

This point, together with some semi-confirmed hints on the BSM physics from rare decays, low-energy measurements, astrophysical observations and cosmological analyses, has motivated one to look for new strategies for DM searches at colliders, and for other DM candidates and DM-related models, like, for example, dark photon, light dark matter (LDM) particles with masses $0.1 \div 10 \mathrm{GeV} / c^{2}$ [212] and many others. Only some of them are briefly discussed in this section.

From the other side, people traditionally believe that such a situation suggests a need for much higher energies. One expects the future 100- TeV collider would be exciting for BSM-searches, including the search for a DM candidate [2].

7.1. Dark photon, dark gauge boson searches. One believes that the dark sector is complicated and could be charged under a new Abelian group $\mathrm{U}^{\prime}(1)$. A relevant light new mediator, the so-called dark photon $A^{\prime}$ (with mass $m_{A^{\prime}}$ ), connects the dark sector to the SM one. The "dark photon" and "dark boson" are typical names of some objects which mediate interaction between the dark sector and the visible one. In fact, any experimental evidence in favor of these dark forces has today nothing to do with detection of galactic true DM particles. Only further model-dependent assumptions can connect these objects with the DM problem.

Results of the special search for the dark photon $A^{\prime}$ in $\pi^{0}$ decays by the NA48/2 experiment at CERN was reported in [213]. One looked for the dark photon via the "signal" decay chain $K^{ \pm} \rightarrow \pi^{ \pm} \pi^{0}, \pi^{0} \rightarrow \gamma A^{\prime}$, and $A^{\prime} \rightarrow e^{+} e^{-}$on the basis of $5 \times 10^{6}$ fully reconstructed $K^{ \pm} \rightarrow \pi^{ \pm} \pi^{0}$, $\pi^{0} \rightarrow \gamma e^{+} e^{-}$decays in the kinematic range $m_{e e}>10 \mathrm{MeV} / c^{2}$ with a negligible background contamination. No signal was observed, and exclusion limits were set on the dark photon mass $m_{A^{\prime}}$ and the mixing parameter $\epsilon^{2}$, which connects dark and visible photons. See also [214].

With the BABAR detector, using $514 \mathrm{fb}^{-1}$ of data collected, an experimental search for the dark photon $A^{\prime}$ in the reaction $e^{+} e^{-} \rightarrow \gamma A^{\prime}, A^{\prime} \rightarrow e^{+} e^{-}, \mu^{+} \mu^{-}$was carried out [215. No significant deviations from SM were observed and upper limits were established on the dark-toreal photon mixing at the level of $\epsilon \simeq 10^{-4}-10^{-3}$ for $0.02<m_{A^{\prime}}<10.2 \mathrm{GeV} / c^{2}$. The range of the parameter space favored by interpretations of the discrepancy between the calculated and measured anomalous magnetic moments of the positive muons ( $g-2$ muon anomaly [216]) was severely constrained. Together with the above-mentioned results from NA48, the BABAR results exclude the entire region favored by the dark-photon scenario for the $g-2$ measurements [217.

The proposal for searching for a dark photon $A^{\prime}$ with mass $10-80 \mathrm{MeV} / c^{2}$ via the decay $\mu^{+} \rightarrow e^{+} \nu_{e} \bar{\nu}_{\mu}+A^{\prime}$ followed by $A^{\prime} \rightarrow e^{+} e^{-}$was formulated for the upcoming Mu3e experiment at the Paul Scherrer Institute [218]. The primary goal of the experiment is "traditional" search for the very rare lepton number violating and SM-forbidden decay $\mu^{+} \rightarrow e^{+} e^{+} e^{-}$. With expected $10^{15}\left(5.5 \times 10^{16}\right)$ muon decays in 2015-2016 (2018 and beyond), the Mu3e collaboration has a 
very good opportunity to reduce substantially the currently unexplored dark photon parameter space, probing the mixing parameter up to $\epsilon^{2} \sim 10^{-7}\left(10^{-8}\right)$.

In searching for a signal of the dark photons in ATLAS at the LHC tightly collimated groups of highly-boosted leptons - lepton-jets — were used [165]. There is essentially no SM background for them. These jets were predicted in [219] and were motivated by a DM model which is consistent with a possible positron excess in cosmic rays. In the model DM is the SUSY LSP, but it can decay into lighter "hidden valley" particles resulting in the production of dark photons which can finally decay into highly-boosted leptons.

Searches were made for muon jets containing 4 or more muons; pairs of muons jets of 2 or more muons; pairs of electron jets of 2 or more electrons. No significant excess of lepton jets was observed over the expected SM background. For broad applicability, limits expressed in terms of the signal cross section times branching ratio were derived for each pair of the dark gauge coupling parameter, $\alpha_{D}$, and $m_{A^{\prime}}$ [220]. Dissipative hidden sector generic DM model was considered in [221, 222].

Another idea to use the lepton-jets for search for light dark force carriers, $Z^{\prime}$-bosons, at the LHC was proposed in [223]. The GeV-scale dark gauge boson $Z^{\prime}$ was discussed [224, 225] in the context of some astrophysical anomalies and the $3.6 \sigma$ deviation from SM in the muon $g-2$ measurement [216]. A scenario, which could be easily probed at the LHC RUN-II, was studied where a top quark was assumed to undergo exotic decay to a $b$ quark and a charged Higgs followed by $H^{ \pm} \rightarrow W^{ \pm}+Z^{\prime}$. The decay products of the dark $Z^{\prime}$ further form a highly collimated lepton-jet. It is believed that the feature could help find the new boson with the $t \bar{t}$ samples.

A possibility for dark gauge $Z^{\prime}$ boson search at LHC was discussed in [226] via registration of events with the $Z^{\prime}$ resonance decay into dilepton pairs in association with large $E_{\mathrm{T}}^{\mathrm{miss}}$. This search channel is considered as a generic probe of $\mathrm{TeV}$-scale dark models with involvement of other dark sector particles. Another example of the dark $Z^{\prime}$ boson search with a collider was considered in [227]. The GeV-scale $Z^{\prime}$ boson, produced mainly from DM final state radiation, decays eventually to hadrons or leptons [228], which form a unique mono- $Z^{\prime}$ jet (or dilepton) signature. This final state contains significant discovery potential, which has not yet been examined in detail by the LHC experiments [228].

The famous muon $g-2$ anomaly [216] still works as the main motivation for the theoretical and experimental papers about light dark bosons of $\mathrm{MeV}-\mathrm{GeV}$ scale which could explain the anomaly. As clearly pointed out, for example in [229], the discussions on the subject are not necessarily linked to the DM physics. Sometimes there is no such a link at all. Nevertheless, the word "dark" is widely used, like dark leptonic gauge $Z^{\prime}$ boson or dark photon $A^{\prime}$, for the very suppressed coupling in contrast to the "bright" photon coupling.

Because of active searches in fixed target experiments and rare meson decays, the popular dark photon model is practically excluded as a possible solution of the $g-2$ problem, unless an invisibly-decaying dark photon mode is considered (see review in [229]). But a severe drawback of the mode is the requirement of very light and low-motivated new DM particles, lighter than the $\mathrm{MeV}-\mathrm{GeV}$ scale of the dark photon itself.

An alternative model with a dark leptonic gauge $Z^{\prime}$ boson based on the gauged lepton number or $\mathrm{U}(1)_{\mathrm{L}}$ symmetry was proposed in [229]. Unlike the dark photon, which couples only to a 
charged particle, the $Z^{\prime}$ boson can couple to SM neutrinos and charged leptons with the same strength. Furthermore, the $Z^{\prime}$ boson mainly decays right into the SM neutrinos and avoids the severe quarkonium decay constraints [230, 231, 215] as it does not couple to quarks.

There are further aspects to verify the $\mathrm{U}(1)_{\mathrm{L}}$ dark leptonic gauge boson model, which include the phenomenology of the exotic leptons required for the gauged $\mathrm{U}(1)_{\mathrm{L}}$, especially for the LHC experiments and implications for the neutrino physics [229].

In fact, the idea of a dark $Z^{\prime}$-boson is very popular (see, for example, recent paper [232]).

Re-analysis of the data from the electron beam-dump experiment E137, conducted at SLAC in 1980-1982, allowed one to obtained new constraints on sub-GeV DM and dark photons [233]. It was assumed that the DM candidates can interact with electrons (via a dark photon). Hence, DM can be produced in the electron-nucleus collisions and can be scattered off electrons in the E137 detector. The expected result could be striking: zero-background signature of a high-energy electromagnetic shower that points back to the beam dump. From non-observation of the signal the E137-result has constrained the possibility that invisibly decaying dark photons can explain the 3.6 $\sigma$ discrepancy between the measured and the SM value of the muon anomalous magnetic moment [216]. The E137 data also have convincingly demonstrated that (cosmic) backgrounds can be controlled and could serve as a powerful proof-of-principle for future beam-dump searches for sub-GeV dark matter scattering off electrons in the detector [233].

A search for a new electrophobic sub-GeV dark boson with a missing-beam-energy method was proposed in [234]. The idea of the search relies on two main assumptions. First, the $Z^{\prime}$ boson can be produced by $\mu$-beam scattering on nuclei $A$ via the reaction $\mu+A \rightarrow \mu+A+Z^{\prime}$. Second, one can "observe" $Z^{\prime}$ by looking for some excess of events with the large missing $\mu$ beam energy in a detector. The missing of the $\mu$-beam energy is expected to be due to the decay $Z^{\prime} \rightarrow \nu \nu$. The authors of [234] believe that the very specific signature and high quality of the muon beams at CERN SPS allows one to reach a sensitivity in coupling constant $\alpha_{d}$, which is three orders of magnitude higher than the value required to explain the $g-2$ anomaly. This looks very promising indeed if a good enough, better sub-MeV-scale, missing beam energy resolution will be achieved experimentally, and one is able to prove independently that the $Z^{\prime}$ boson was indeed produced before it decayed invisibly.

Preliminary estimates of the expected light hidden photon signal rate were presented in [235] with respect of the recently proposed fixed target SHiP experiment [236] exploiting the CERN SPS beam of $400 \mathrm{GeV}$ protons.

An extended program of different ways for the dark photon search (including invisible Higgs boson decays, etc.) with future hadron colliders was considered in [237].

Similar missing energy-momentum approach was described in [238, as authors claimed, for detection of $\mathrm{DM}$ and other invisible particles with mass below $1 \mathrm{GeV} / c^{2}$ in fixed-target accelerator experiments. The main idea is to exploit missing energy-momentum measurements and other kinematic features of fixed-target particle production. Several new beam-dump experiments are already aimed to produce light DM candidates and "observe" their scattering in downstream detectors [239, 240, 241, 233, 242. A typical setup is given in Fig. 50. One believes that this technique, with $E_{\text {missing }}=E_{e}^{i}-E_{e}^{f} \simeq E_{B}$, allows one to discover this kind of events, which under severe assumptions (see sections 6 and 8) can be interpreted as observation of light DM candidates. Furthermore, the method relies on a very small re-scattering probability and it 


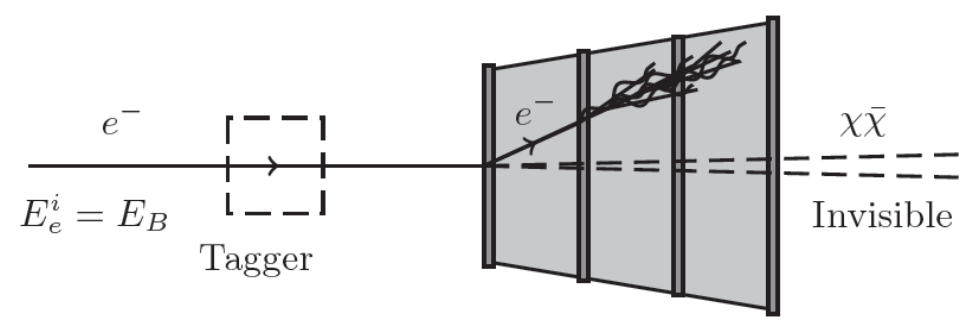

Figure 50. Scheme of the fixed-target dark photon search experiment. A single beam electron first passes through an up-stream tagger to fix its energy. Then it enters the target/calorimeter and emits an $A^{\prime}$, which decays somewhere invisibly into a dark pair $\chi \bar{\chi}$ and carries away most of the beam energy. In the final state one has only the electron with very small energy $E_{e}^{f} \ll E_{B}$. From [238].

seems challenging to reach relevant sensitivity. The sensitivity requires identification of "the DM production events" solely by their kinematics, which in fixed-target electron-nuclear collisions is believed to be quite distinctive [243.

Under the assumption that the above-mentioned events are due to an invisibly decaying $\mathrm{MeV}-\mathrm{GeV}$-scale dark photon $A^{\prime}$, this approach can improve present constraints by $2-6$ orders of magnitude over the entire $m_{A^{\prime}}$ range, and sensitivity as low as $\epsilon^{2} \sim 10^{-14}$ can be reached [238].

The belief in a powerful potential of electron-beam fixed-target experiments for discovery of DM and other new WIMPs in the MeV-GeV mass range [239, 240, 241, 233, 242] is realized in a new proposal for a Pilot Dark Matter Search at Jefferson Laboratory [244]. The physics potential of such an experiment was discussed and highlights of its unique sensitivity to inelastic "exciting" DM and leptophilic DM scenarios were stressed. The first of these seems kinematically inaccessible in traditional direct detection experiments.

The main principles of production and detection of these particles are depicted in Fig. 51 . The first stage of this program can be realized at Jefferson Laboratory using the existing plasticscintillator detector downstream of the Hall D electron beam dump.

Letter of Intent for Beam-Dump eXperiment (BDX) at Jefferson Laboratory 242] is a concrete realization of the DM program. The BDX will look for the $\mathrm{MeV}-\mathrm{GeV}$ WIMP DM candidates with a $1 \mathrm{~m}^{3}$ segmented plastic scintillator detector placed downstream of the beam-dump at one of the high intensity JLab experimental Halls. Up to $10^{22}$ electrons-on-target were expected in a one-year period. The BDX will be sensitive to WIMP-nucleon elastic scattering at the level of a 1000 counts/year, with very low threshold recoil energies $(\sim 1 \mathrm{MeV})$. Expected sensitivity to WIMP-electron elastic scattering and/or inelastic WIMP would be below 10 counts/year after severely reduced backgrounds.

An existing $0.036 \mathrm{~m}^{3}$ detector prototype based on the same technology will be used to validate simulations with background rate estimates, driving the necessary $R \& D$ towards an optimized detector. A fully realized experiment would be sensitive to large regions of DM parameter space, exceeding the discovery potential of existing and planned experiments by two orders of magnitude in the $\mathrm{MeV}-\mathrm{GeV}$ DM mass range [242].

Some comments about this typical proposal are in order. First, its goal is unusual events with large undetected energy (momentum) comparable to the initial electron beam energy. To see the events, one needs very good sensitivity and very strong background reduction. The 
a)

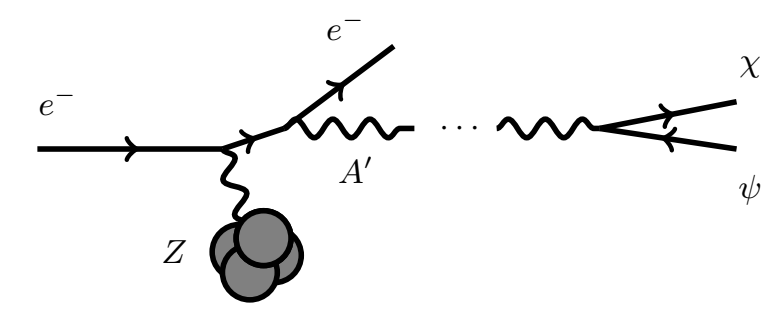

b)

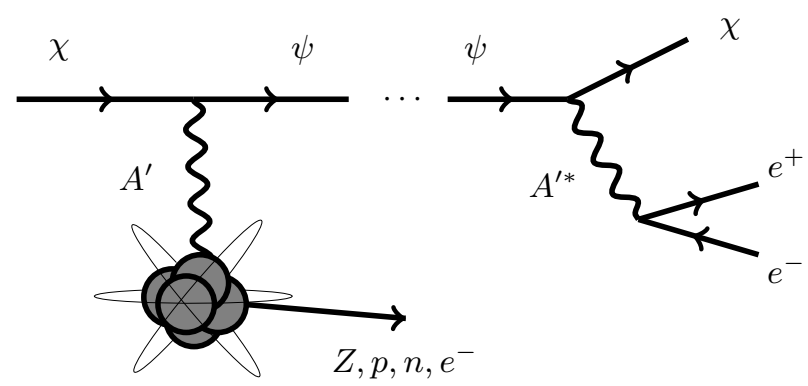

FiguRE 51. a) Fermionic DM pair production via issuing $A^{\prime}$ boson during electronnucleus collisions. In the generic scenario with Dirac and Majorana masses for dark sector fermions, the $A^{\prime}$ mediator couples off-diagonally to the mass eigenstates $\chi$ and $\psi$. b) Detector scattering via $A^{\prime}$ exchange inside the detector. If the mass splitting between dark sector states is negligible, both the incoming and outgoing DM states in the scattering process are invisible and can be treated as the same particle. For order one (or larger) mass splittings, $\chi$ can upscatter into the excited state $\psi$, which promptly decays inside the detector via $\psi \rightarrow \chi e^{+} e^{-}$. This process yields a target (nucleus, nucleon, or electron) recoil $E_{R}$ and two charged tracks, which is a distinctive, low background signature, so nuclear recoil cuts need not be limiting. Processes analogous to both a) and b) can also exist if DM is a scalar. From 244.

most probable result of the search will be non-observation of any of the events. The next point is interpretation of the results. It is no way to prove that the $A^{\prime}$ boson was indeed produced before it decayed invisibly. Here one must make a strong assumption on the subject. In fact, the assumption concerns right the point one should prove experimentally. Of course, some limit can be set from almost any measurement for almost any measurable value, if one clearly writes down all assumptions. The main question is their adequateness and correctness.

Finally, such an experiment is much farther from the solution of the DM problem if compared with the LHC DM search program. With the LHC one tries to see a real DM candidate, but here one can see a particle that only can be or cannot be a DM-SM messenger, nor ever a DM candidate, to say nothing about the true DM galactic feature, like the annual signal modulation. Much more model dependence is needed to connect this search with the DM problem.

Another step of the DM program at Jefferson Laboratory is DarkLight experiment [245] aimed at a precision search for New Physics at low energies. The famous dark photon with $m_{A^{\prime}}=10 \div 100 \mathrm{MeV} / c^{2}$ was the main motivation. The DarkLight will precisely study the $e p \rightarrow e p+e^{+} e^{-}$reaction via detection of the final state scattered electron, recoiled proton, and $e^{+} e^{-}$pair. The signal would be the reaction $e p \rightarrow e p+A^{\prime}$ followed by $A^{\prime} \rightarrow e^{+} e^{-}$. To this end a windowless gas target of molecular hydrogen will be irradiated by the $100 \mathrm{MeV}$ electron beam of intensity $5 \mathrm{~mA}$. Phase-I of the experiment was funded and one expects to take data 
soon. Complete phase-II is under final design and could run within two years after phase-I is completed. The DarkLight experiment to be decisive requires development of a new technology for beam, target, and detector.

Contrary to the experiment aimed at the invisible dark photon decay, search of the dark photon decay into a well measurable $e^{+} e^{-}$-pair looks much more promising and better motivated.

A proposal to obtain valuable constraints on the dark photon $A^{\prime}$ as a true DM candidate from results of traditional direct DM search experiments was given in [246]. The absence of an ionization signal in Xe direct detection experiments was used to place a very strong constraint on the dark photon mixing angle, down to $O\left(10^{-15}\right)$, under the assumptions that the dark photons are long-lived vector states with $0.01-100 \mathrm{keV} / c^{2}$ masses and they comprise the dominant fraction of DM in the Universe.

Here $A^{\prime}$ photon plays the role of the super-weakly-interacting DM and has certain advantages over axion-like-particle DM with respect to direct detection [246].

7.2. Exotic DM search with colliders. The observation of the SUSY or any new BSM model and determination of the DM properties will require establishing the predicted particle spectrum [247]. One can mention several ways of investigating the DM problem at the LHC, additional to the traditional $E_{\mathrm{T}}^{\text {miss }}$-method. For example, one can study cascade decays of colored (SUSY) particles, vector-boson-fusion productions of non-colored (SUSY) particles, mono-jet and direct stop production searches [247].

One believes that some fraction of possible DM candidates can couple only to leptons (leptophilic DM [248, 249]) and therefore were very weakly constrained from the LHC and direct DM detection searches. In such models the interaction is described by effective four-lepton contact operators, which can be probed in $e^{+} e^{-}$-collisions.

The precise data from LEP was used in [250] to derive limits on this leptophilic DM in a model-independent EFT framework. The bounds turn out to be very competitive with exclusion curves obtained from LHC data from mono-photon events with large $E_{\mathrm{T}}^{\text {miss }}$. Furthermore, the future ILC (International Linear Collider) data allow one to set the strongest limits on TeV-scale leptophilic DM candidates [250].

The potential of the ILC for the solution of the DM problem was studied in [251]. Within the EFT approach the reach of the ILC was compared with that of other searches. Low mass WIMPs are as usual a key feature for a collider search including the ILC. If it happens that the WIMP can only couple to leptons or only spin-dependently, the ILC could be especially useful to study and constrain such a set of models.

The next opportunity for a future $e^{+} e^{-}$collider to help with solution of the DM problem was considered in 252] and was motivated by an excess of energetic photons observed in the center of our Galaxy with the Fermi-LAT satellite [253, 254]. It was shown that if the DM candidates are assumed to be the (Majorana or Dirac) fermions $\chi$ and couple to a $Z^{\prime}$ boson, one could observe a remarkable DM-related signal at a $\mathrm{TeV} e^{+} e^{-}$collider via the process $e^{+} e^{-} \rightarrow \chi \chi$ +photon. This result relied on the ability to use highly polarized beams to eliminate the background $e e \rightarrow \nu \nu \gamma$ process due to $W$ exchange. It also requires an optimized setup to fully eliminate the contamination from $e e \rightarrow e e \gamma$. Finally, prospects for the DM search at the $e^{+} e^{-}$colliders were presented. 
The overall conclusion of [252] was that a BSM mediator, for instance a $Z^{\prime}$ or extra Higgs bosons, is needed to interpret the Fermi-LAT DM evidence. If true, these interpretations predict scenarios which could already be tested at the LHC while a $\mathrm{TeV} e^{+} e^{-}$-collider should provide an essential tool for a precise measurement of the parameters of the BSM resonances.

An $e^{+} e^{-}$collider was also proposed to look for production of a Higgs boson recoiling from a massless invisible system [255]. One believes this can be a quite distinctive signature of the Higgs boson creation in association with a massless dark photon $e^{+} e^{-} \rightarrow H+A^{\prime}$. Dark photons can acquire effective couplings to the Higgs boson via loop diagrams. The signal and corresponding backgrounds for $H \rightarrow \bar{b} b$ were analysed, and the ILC [256] and FCC-ee [257] sensitivities were estimated in a model-independent way [255].

"Untraditional" usage of the positron beam of the DA $\Phi$ NE linac at the Laboratori Nazionali di Frascati was proposed [258] for dark photon search in Positron Annihilation into Dark Matter Experiment. The PADME experiment will search for the dark photon $A^{\prime}$ in the $e^{+} e^{-} \rightarrow \gamma A^{\prime}$ process in a positron-on-target experiment configuration. After one year a sensitivity in the relative interaction strength $\epsilon^{2}$ down to $10^{-6}$ is achievable, in the mass region from $2.5<$ $m_{A^{\prime}}<22.5 \mathrm{MeV} / c^{2}$.

The DA $\Phi$ NE collider with the detector KLOE was also used to search for the so-called Higgsstrahlung process $e^{+} e^{-} \rightarrow A^{\prime}+h^{\prime}$, where $A^{\prime}$ is the dark photon, and $h^{\prime}$ is the dark Higgs boson, which decays invisibly. No evidence for the signal was observed and, in particular, upper limits on the kinetic mixing parameter $\epsilon$ in the range $10^{-4} \div 10^{-3}$ were established [259, 260].

Assuming prompt decays of the dark photon $A^{\prime}$ and the dark Higgs boson $h^{\prime}$, the Belle collaboration also performed [261] a search for their production in the Higgs-strahlung channel, $e^{+} e^{-} \rightarrow A^{\prime} h^{\prime}$, with $h^{\prime} \rightarrow A^{\prime} A^{\prime}$. Analysis of the full set of $977 \mathrm{fb}^{-1}$ Belle data gave no significant signal evidence. The $90 \% \mathrm{CL}$ upper limits were obtained on the branching fraction times the Born cross section, $\mathrm{BR} \times \sigma_{\mathrm{Born}}$, on the Born cross section, $\sigma_{\mathrm{Born}}$, and on the dark photon coupling to the dark Higgs boson times the kinetic mixing between the SM photon and the dark photon, $\alpha_{D} \times \epsilon^{2}$. These limits improve upon and cover wider mass ranges than previous experiments. For $\alpha_{D}=1 / 137, m_{h^{\prime}}<8 \mathrm{GeV} / c^{2}$, and $m_{A^{\prime}}<1 \mathrm{GeV} / c^{2}$ Belle excluded values of the mixing parameter $\epsilon$ above $8 \times 10^{-4}$ [261].

Further discussions of exotic DM search program with $e^{+} e^{-}$colliders can be found in, for example, [262, 263].

For a collider experiment a well-known challenge is to determine the mass of an undetected particle due to the under-constrained kinematics with two missing particles in an event. It is especially difficult at hadron colliders because of the further unknown kinematics of initial partons. There are many attempts to determine the missing particle mass at the LHC, such as endpoint methods (see for example [264, 265, 266]), polynomial methods (see for example [267, 268, 269]), $M_{T 2}$ methods (see for example [270, 271, 272, 273]), and the matrix element method (see for example [274, 275]).

In view of the scientific program of the future high energy lepton collider, in particular future DM search, the problem of how to determine the mass of undetected DM candidates through the so-called antler topology process given in Fig. 52 was specially studied in [20. The antler decay diagram [276] was used for investigation of a resonant decay of a heavy particle $D$ into 


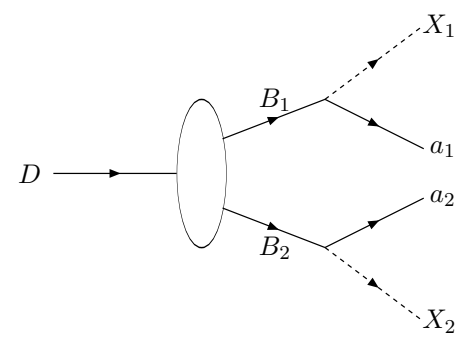

Figure 52. The antler decay diagram of a heavy particle $D$ into two visible particles $a_{1}$ and $a_{2}$ and two invisible particles $X_{1}$ and $X_{2}$ through on-shell intermediate particles $B_{1}$ and $B_{2}$. From [20].

two intermediate particles ( $B_{1}$ and $B_{2}$ ), followed by each $B_{i}$ 's decay into a missing particle $X_{i}$ and a visible particle $a_{i}$. It was found that the resonant decay through this diagram develops cusps in some kinematic distributions and the cusp positions along with the endpoint positions can determine both the missing particle mass $m_{X}$ and the intermediate particle mass $m_{B}$.

A lepton collider has an advantage due to the well-defined initial state with fixed center-mass energy and center-mass frame, which allows one to study many new physics processes by means of the above-mentioned antler topology $e^{+} e^{-} \rightarrow B_{1} B_{2} \rightarrow X_{1} a_{1}+X_{2} a_{2}$. It was found in [20] that the cusp method appeared to be more stable than the commonly considered energy endpoints against realistic factors (initial state radiation, acceptance cuts, detector resolution) and is very efficient for measuring the missing particle mass.

Considering as an example the pair production of scalar muons (smuons) as the MSSM process that satisfies the antler topology, it was shown that at the $500 \mathrm{GeV}$ ILC mass determination with precision of $0.5 \mathrm{GeV} / c^{2}$ can be achieved for smuons with a leptonic final state.

This method can be useful for determination of mass spectrum of any BSM model. As an expected by-product of the procedure one can obtain very useful indication of masses of the invisible DM candidates. Nevertheless the proof of true DM nature of the candidates still remains inevitable.

There is a proposal to study mono-jet and $E_{\mathrm{T}}^{\text {miss }}$ signature from $\gamma p$ collisions at the LHC in context of the DM problem [277]. In fact, photoproduction of jet $+E_{\mathrm{T}}^{\mathrm{miss}}$ final states was simulated in a model independent EFT framework assuming a typical LHC forward detector. Rather good prospects for constraining the couplings of the quarks to the WIMP DM candidates were obtained on the basis of the main reaction $p p \rightarrow p \gamma p \rightarrow p \chi \chi+$ jet for an integrated luminosity of $200 \mathrm{fb}^{-1}$ as a function of the forward detector acceptance.

A very light dark photon from the hidden sector could couple to SM particles and could give a signal via $\nu e$-scattering experiments if the dark photon is the gauge field of a $\mathrm{U}(1)$ group. This will allow a direct coupling with neutrinos. The new interactions due to existence of the $A^{\prime}$ boson whose couplings do not contain derivatives lead to the differential cross section being proportional to $1 / T^{2}$, which makes low energy $\nu$-experiments sensitive to the $A^{\prime}$ boson search in the low mass region. Hence low energy neutrino experiments aimed to measure neutrino-nucleus coherent scattering as well as neutrino magnetic moment has an advantage in searching for the $A^{\prime}$ boson, with mass located much below the electroweak scale. For the higher mass region of $A^{\prime}$ boson, neutrino experiments with higher incident energy have better sensitivity [278]. 
It is perhaps not meaningless to recall a "crazy" idea about usage of a very intensive beam of accelerated particles or ions with large enough energy for cold DM particle search [279, 280]. The detection strategy was in some sense reversed to the traditional direct DM method. The non-relativistic and practically motionless galactic cold DM particles were considered as a target permanently distributed in space around a collider. During all working time of the collider this target is irradiated by an intensive beam of relativistic particles. One should only wait for a moment when a beam particle suddenly knocks one of the DM particles, producing a very unusual event with matter and energy release from "an empty place".

Unfortunately, numerical estimations showed that one should wait almost as long as man's life (due to very low local relic density). Nevertheless, future very intensive beams from CERN's 100-TeV Hadron Collider [281, International Facility for Antiproton and Ion Research (FAIR) [282], or Nuclotron based Ion Collider fAcility (NICA) [283] could perhaps bring some sense to the idea.

7.3. Exotic DM search with accelerators. Search for accelerator-produced light WIMP DM candidates was carried out with MiniBooNE [284] using $1.86 \times 10^{20}$ protons with $8.9 \mathrm{GeV} / c$ momentum, directed to a steel beam-dump $50 \mathrm{~m}$ down-stream.

As already mentioned, due to nuclear recoil measurements, current direct DM detection experiments have rather low sensitivity to WIMP masses below about $1 \mathrm{GeV} / c^{2}$. Despite the mandatory improvement of the direct detection sensitivity, it seems reasonable to use an accelerator for production of a "beam" of relativistic (boosted) low-mass WIMP DM candidates, which further can be detected with a proper neutrino detector, due to the similarity of the WIMP- and $\nu$-interaction signatures in the detector (weak neutral current events). Running MiniBooNE in the beam-dump mode reduced the neutrino background by having the beam hit a steel beam-dump instead of hitting the Be target.

The MiniBooNE experiment, located at FNAL on the Booster Neutrino Beamline, has already accumulated the largest collection of $\nu$ and $\bar{\nu}$ samples. Being well understood, the setup is well suited for detection of events which could be generated by interaction of accelerator-produced relativistic low-mass WIMPs. Preliminary analysis did not show signal events. Final results are expected at the end of 2015.

Nevertheless, one should keep in mind that in this case the accelerator produced WIMPs should have lifetime $c \tau \simeq 0.49 \mathrm{~km}$ to reach unchanged the detector from the collider.

Another idea to look for high energy scattering of "dark Dirac fermions" from nuclei was considered in [285]. One assumes that when the DM candidate particle $\chi$ is light enough to escape the traditional direct detection, a promising way to look for the $\chi$ is a fixed target experiment [286, 287], where $\chi$ 's can be pair (hadro)produced by an $s$-channel exchange of a light vector boson.

Being sufficiently weakly interacting the $\chi$ paricle can pass through shielding (that screens out strongly interacting products) and can be detected in a neutrino-like detector by means of interaction similar to neutrino neutral current scattering. The advantage of a fixed target experiment over a colliding one is the much higher luminosity, which becomes decisive when one searches for extremely rare events. 
This idea was tested with the Fermilab experiment E613 data, and limits on a "secluded" (mediator is lighter than $\chi$ ) DM scenario [286, 288] was obtained.

A recent review, concerning new limits on light hidden sectors from fixed-target experiments can be found in [289].

The idea to use a fixed-target neutrino experiment technique for the laboratory search for light weakly interacting dark sectors was further discussed in [290. It was shown that the DAEdALUS source setup - an $800 \mathrm{MeV}$ proton beam impinging on a target of graphite and copper - can improve the present bound on the dark photon $A^{\prime}$ (produced here mainly from $\pi^{0}$ decays) by an order of magnitude over much of the accessible parameter space for light DM $\chi$ (produced via $A^{\prime} \rightarrow \chi \bar{\chi}$ ) when paired with a suitable neutrino detector such as LENA [291] (signal process for detection is $\chi e^{-} \rightarrow \chi e^{-}$).

It was shown in [290] that DAEdALUS was sensitive to DM particles produced from offshell dark photons and that fixed-target experiments had sensitivity to a much larger range of heavy dark photon masses than previously thought. The mechanism for the DM production and detection through a dark photon mediator was reviewed together with the discussion of the beam-off and beam-on backgrounds, and present the sensitivity to dark photon kinetic mixing for the DAEdALUS/LENA setup in both the on- and off-shell regimes.

It appears that intensity frontier experiments like DAEdALUS in conjunction with a large underground neutrino detector such as LENA will have unprecedented sensitivity to light (sub-50 $\mathrm{MeV}$ ) DM $\chi$, light (sub-400 MeV) dark photons $A^{\prime}$, and other light weakly interacting particles.

One agrees with the statement from [290] that both neutrino and accelerator fixed-target DM search experiments share essentially the same signals and backgrounds (though often wellseparated kinematically), and this fact suggests exciting opportunities for symbiosis between BSM and neutrino physics in the coming years.

Another possibility of using a large volume neutrino detector for detection of relativistic DM particle candidates was discussed in [292], where the Sun was assumed to be a source of these boosted DM particles [293]. Their arrival direction from the Sun can be used as a important signature. To arrange the energetic DM fraction, one proposed a scenario where thermal DM can be efficiently captured in the Sun and can annihilate into another sort of DM, right the boosted one. At least in models with a multi-component (or non-minimal) structure of DM sector (for example [59]), annihilations of viable thermal relic DM with masses $1-100 \mathrm{GeV} / \mathrm{c}^{2}$ can produce other stable DM particles with moderate Lorentz boosts.

The detection of this relativistic DM-like particle is expected to be due to its interaction with a target proton (or nucleus) in some (very) large volume terrestrial detector, resulting in an energetic proton recoil track pointing towards the Sun. Cherenkov-radiation-based detectors Super-Kamiokande [294] and Hyper-Kamiokande [295] were considered as examples for sensitivity study.

In particular, one found that by means of spin-dependent interaction the considered boosted DM candidates could produce detectable signals with sensitivity comparable to DM direct detection experiments. Future large-volume liquid Argon neutrino detector [296] based on ionization signals or neutrino telescopes [297, 298, 299, 31] may significantly extend the sensitivity.

Concluding this point, one agrees with 292] that the possibility of detecting some energetic fraction of unknown weakly interacting particles, which have a habit to arrive on the Earth from 
the Sun can be very crucial for understanding the DM sector structure. The very idea to use large-volume neutrino (or proton-decay) detectors for investigation of the DM problem looks particularly intriguing.

7.4. Other exotic DM searches. Constraints on dark forces from the electron-positron colliders (B factories), fixed-target experiments and flavor-physics measurements at hadron colliders were discussed in [300]. The basic models where DM sector interacts with SM particles via mediation of new dark vector and scalar bosons with masses in the $\mathrm{MeV}$-to-GeV range are reviewed. The typical processes are given in Fig. 53. Recently dedicated searches for these

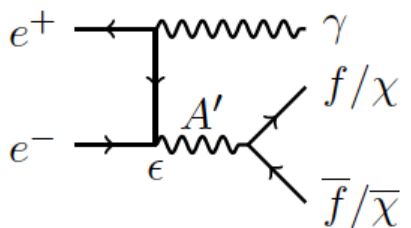

(a)

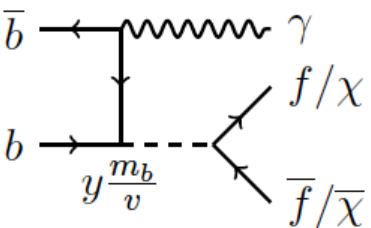

(b)

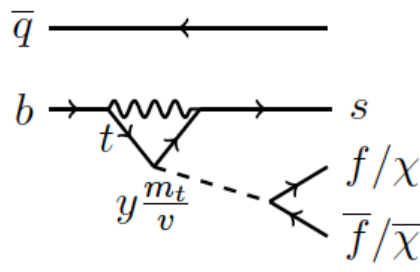

(c)

Figure 53. Production of (a) dark photon in $e^{+} e^{-}$-collisions, (b) dark Higgs $\phi$ in $Y(b \bar{b})$ decay, and (c) dark Higgs in penguin B decay. The dark photon $A^{\prime}$ or dark Higgs (dashed line) is shown decaying into a pair of SM fermions $f \bar{f}$ or invisible dark-sector fermions $\chi \bar{\chi}$. From [300].

low-mass bosons have been conducted, and rather tight limits on the parameter spaces of the relevant new-physics models have been established. Constraints from current measurements rule out significant regions of model-parameter space. Higher sensitivities will be achieved by the next generation of B-factory and fixed-target experiments, as well as by RUN II of the LHC.

One believes that these vector and scalar dark bosons couple to stable DM particles, and all together constitute the so-called dark sector.

Invisible decays of heavy quarkonium states can be considered as another source of complementary information for constraining properties of possible light DM candidates. Contrary to the invisible decays of the not-yet-observed dark photon $A^{\prime}$ or dark gauge boson $Z^{\prime}$, invisible decays of such well-observed objects like $\Upsilon(\mathrm{nS})$ looks much more reasonable and reliable. Furthermore, one believes that in contrast to DM-mono-jet searches at high energy colliders, Band charm factories are more suitable for light DM candidate search with a lower mass mediator [212].

Using data from high intensity electron-positron colliders and assuming that the light DM candidate couples universally to all quarks, new constraints on the properties of the light DM candidates were obtained from the analysis of invisible quarkonium decays in [212]. The analysis was based on the results of the searches for $\Upsilon(1 \mathrm{~S})$ invisible decays performed by Belle [301] and BaBar [302] operating at the energy of $\Upsilon(3 \mathrm{~S})$ resonance. The transition $\Upsilon(3 \mathrm{~S}) \rightarrow \pi^{+} \pi^{-} \Upsilon(1 \mathrm{~S})$ was used to detect invisible $\Upsilon(1 S)$ decays and to reconstruct the presence of the $\Upsilon(1 S)$ from the $\Upsilon(1 \mathrm{~S})$ peak in the recoil mass distribution, $M_{\text {rec }}$, by tagging $\pi^{+} \pi^{-}$pairs with kinematics $M_{\text {rec }}^{2} \equiv s+M_{\pi \pi}^{2}-2 \sqrt{s} E_{\pi \pi}^{*}$, where $M_{\pi \pi}$ is the invariant mass of the pion system, $E_{\pi \pi}^{*}$ is the energy of the pion system in the center-of-mass frame of the $\Upsilon(3 \mathrm{~S})$, and $\sqrt{s}=10.3552 \mathrm{GeV}$ is 
the $\Upsilon(3 S)$ resonance energy. This tagging allowed one to make sure of the existence of the $\Upsilon(1 S)$ particle (as an initial state for the invisible decay $\Upsilon(1 \mathrm{~S}) \rightarrow \chi \bar{\chi}$ ). Similar searches for invisible decays of $J / \Psi$ were based on the transition $\Psi(2 \mathrm{~S}) \rightarrow \pi^{+} \pi^{-} J / \Psi$.

The experimental limits $(90 \% \mathrm{CL})$ on the branching ratios for the invisible decays are $\mathrm{BR}(\Upsilon(1 \mathrm{~S}) \rightarrow$ invisible) $<3.0 \times 10^{-4}$ from the BaBar collaboration [302] and $\mathrm{BR}(J / \Psi \rightarrow$ invisible $)<7.2 \times 10^{-4}$ from the BES collaboration 303. The SM contribution to these invisible decay modes were found to be negligible [304]: $\mathrm{BR}(\Upsilon(1 \mathrm{~S}) \rightarrow \nu \bar{\nu})=9.85 \times 10^{-6}$ and $\mathrm{BR}(J / \Psi \rightarrow \nu \bar{\nu})=2.70 \times 10^{-8}$.

In the framework of a low-energy EFT for each of the contact operators (Table 1) relevant to the invisible $\Upsilon(1 S) \rightarrow \chi \bar{\chi}$ decay exclusion limits for the spin-independent and spin-dependent $\chi$-proton cross section were calculated [212] and presented in Fig. 54 .


Figure 54. Bounds on the $\chi$-proton spin-independent (left panel) and spin-dependent (right panel) scattering cross section as a function of mass $m_{\chi}$. The $\chi$ couples universally to quarks through the indicated effective contact operator. The labeled exclusion curves indicate $90 \%$ CL bounds from limits on invisible decays of $\Upsilon(1 \mathrm{~S}), 95 \%$ CL bounds from Fermi-LAT constraints on DM annihilation in dwarf spheroidal galaxies, and 90\% CL bounds from mono-jet searches (CMS [195, 167] and ATLAS [151]). The DAMA/LIBRA [305], CRESST II (95\% CL) [79], CoGeNT [119], CDMS II (Silicon) 81], SuperCDMS [82, LUX [73], SIMPLE [91, PICASSO [87, and COUPP [173] 90\% CL signal regions are also shown. From [212].

One can see that the invisible- $\Upsilon(1 \mathrm{~S})$ bounds are sensitive to a low $\chi$-mass range significantly below the thresholds of current direct DM detection experiments and complement bounds obtained from $\gamma$-ray searches of dwarf spheroidal galaxies and from mono-X searches at hadron colliders. Unfortunately, a question of applicability of a very low mass region for true DM candidates is still open.

It is worth noting that since the $\Upsilon(\mathrm{nS})$ states are non-relativistic, their invisible decays can be sensitive to the DM candidate interaction with non-relativistic quarks. These searches have the same footing as the direct DM searches, contrary to the LHC searches with highly relativistic quarks and gluons. 
Ignoring of DAMA/LIBRA results forces one to look for any theoretical explanation of signal absence in other direct DM experiments, provided pure experimental explanations of the absence are also ignored. On the way a lot of different DM models appear. For example, such a model was considered in [306], where a DM candidate with a mass in $0.1-1 \mathrm{TeV} / c^{2}$ interval was coupled to the SM particles via only one vector boson. The key assumption of this exotic model is that the DM candidate mass is taken to be around $1 / 2$ of the mediator mass. Therefore one has a resonant enhancement of the DM-SM interaction and should strongly reduce the couplings to have the correct relic abundance. The main by-product of this procedure is a very low cross section and a zero result of direct detection. Prospects for verification of the model with the LHC, CTA [307, 25] and AMS [308] observations of the Galactic center were also discussed [306].

Another exotic simplified model of fermionic DM was considered in [309]. The DM couples exclusively to the right-handed top quark via a renormalizable interaction with a color-charged scalar. The relic abundance of this DM was computed and constraints were placed on the model parameter space followed by the discussion of prospect for direct detection. Furthermore, detailed analysis for the production of the DM candidates at the LHC was performed. Several kinematic variables were proposed that allow extraction of a clean signal and reduction of the parameter space of this model during the LHC RUN II. The possibility of detecting this type of DM via its annihilations into $\gamma$-rays was also studied. Another idea to use the top quark for DM search with a collider was also considered in [310].

One must say a word about an axion, which was proposed to explain an anomaly in quantum chromodynamics [311. The electromagnetic signatures of axions have long been sought in the laboratory experiments without any success. String theory suggests an ultralight axion that would be so-called "warm" DM. The mixture of cold and warm DM components (including neutrinos) might resolve some tensions of pure cold DM scenarios. For example, it could explain why there are fewer dwarf galaxies than cold DM predicts [2].

There are exotic proposals to search for different DM candidates through oscillations in the fine-structure constant using atomic spectroscopy [312], atoms clocks [313], and laser and maser interferometry [314]. Search for composite Dark Atoms is discussed in [315, 316, 317, 318].

Finally, there is a common belief [2] that a much broader categories of DM particles (substances) should be sought. For example, one can give up DM neutrality and allow DM to carry a small electric charge or possess some internal states like electron levels of an atom. Precision helioseismology could detect small changes in the solar surface oscillations due to clouds of "millicharged" DM particles scattering off electrons in the solar plasma. More spherical DM haloes of distant galaxies could be measured by means of gravitational lensing, provided the DM particles can interact electromagnetically, and so on.

Concluding this section about exotic DM search programs, one should first stress that nowadays it is a very hard job to make an attempt to produce a complete review on the subject. Almost every day a paper with a new DM model can be found in arXiv. An obviously incomplete list of recent eprint papers includes [319, 320, 321, 322, 323, 324, 325, 326, 327, 328, 205, 329, 206.

Furthermore, strictly speaking, very few of the models and/or searches discussed (and not yet) in the section have something real to do with practical detection of true DM particles, constituting the galactic DM halo of our Galaxy. 


\section{Discussion}

8.1. Effective Field Theory and a bit beyond. The scale of validity of the EFT, in particular the kinematic region where the EFT approach for collider WIMP pair production breaks down, has been discussed from the very beginning [144, 330, 145, 331, 332, 333, 146, 334, 335, 148, 336, 337, 338, 339, 147, 207, 211, 340, 341, 342, 343, 344. One of the recent discussion of the problem can be found in the Appendix of [153] where the region of validity of the EFT approach was studied under various assumptions about the underlying new physics.

Very sophisticated attempts to remedy the core of the "model-independent" conception of the EFT with help of a set of "simplified models" can be found, for example, in [331, 345, 346, 347, 182, 348, 349, 350, 351, 352, 353, 354, 355, 211, 356. It is difficult to add something quantitatively new into both of these considerations.

Nevertheless some general comments concerning this situation are in order.

- Indeed, due to complexity of the DM problem one has to incorporate as much as possible useful information, which could help one to solve the problem. On the way the complementarity of all experimental searches for the DM (discussed in section 2) looks inevitable. To arrange quantitatively this complementarity one needs an approach which could describe all relevant observables with a common set of parameters. Only in this case constraints from one experiment can be connected with and/or applied to results of another one.

- There is a common belief (or prejudice) that such an approach must be as much modelindependent as possible. To fulfill this requirement the EFT was proposed and well developed [145, where a set of Lorentz-invariant 4-point effective operators was collected for description of all possible (and, perhaps, not-possible) DM-vs-SM couplings. For example, in SUSY models the tensor operators look very unusual, or irrelevant at all.

The complementarity goal was reached using the same operators in $s^{-}, t-, u$-channels (as in Fig. 1. section 22. It was believed that the EFT had the undeniable advantage of being independent of the plethora of models of DM [148]. Therefore one got a possibility of putting the WIMP constraints from the LHC on the same ground (in the same plot) with the exclusion curves from the direct and indirect DM search experiments. Nevertheless, it is still unclear how this model-independent comparison and/or competition could help one to solve the DM problem.

Furthermore, in the case of success (an observation of a DM candidate with some/all techniques) one must incorporate observed properties of the DM candidate into a well-developed modern (or very new) theory beyond SM. The above-mentioned model-independent results, if being appropriate, should be inevitably incorporated into the new model and therefore get clear model dependence.

- If one forgets about inner problems of the EFT, some non-comfortability concerning modelindependence of the approach still survives. Indeed, one has 24 different operators (Table 1 ) with 24 different $M_{*}$ scale parameters which appeared to depend rather differently on the mass of the DM candidate $m_{\chi}$. Furthermore, these operators are not connected with each other and are used "one-in-time" - one does not know their interconnection coefficients. Do they interfere constructively or destructively?

This situation does not look better than, for example, in the MSSM, where one has few free parameters for complete numerical descriptions of all above-mentioned observables. The 
parameter manyfold (arbitrariness) of the MSSM is unconsciously substituted by the functional manyfold (arbitrariness) of the EFT in selection of a relevant number of the effective operators.

With 24 above-mentioned $M_{*}-m_{\chi}$ dependences situation looks worse. A rhetorical question could arise - why did not the LHC people include all 24 operators in their analysis? Maybe the people suspected intuitively something wrong with them?

- Too many assumptions of the EFT approach were discussed (in previous sections). In particular, the key assumption concerning the only one SM-DM operator looks far from being reasonable. This is not necessarily the case for the electroweak interaction which has a $V-A$ structure [149]. Furthermore, it is very crucial that there are invisible decays of the next-tolightest (or next-to-next-to-LSP) SUSY particle (sneutrino $\rightarrow \chi+\nu$ ) in many promising SUSY models.

- It was already many times stressed that validity of the EFT results depends on the momentum transfer (through quarks), $Q$, which should be below the energy scale of the underlying interactions $Q<m_{V}$ [148]. With ultraviolet completion $M_{*}=m_{V} / \sqrt{g_{q} g_{\chi}}$ and perturbative regime of the couplings one can obtain, for example, the validity requirements $Q_{\mathrm{D} 1}<4 \pi\left(M_{*}^{3} / m_{q}\right)^{1 / 2}$, $Q_{\mathrm{D} 9}<4 \pi M_{*}, Q_{\mathrm{C} 1}<4 \pi M_{*}^{2} / m_{q}[183$. These $Q$-limits are very different and strongly depend on the type of operator (D1, D9, or C1) and on the details of the relevant parton energy and its distribution.

- Furthermore, one can recall [211] that the rather small expected WIMP-signal rate implies that the scales $M_{*}$ are often smaller than the typical energy of the parton collisions $(\sqrt{s})$. As a result, the interpretation of LHC data in terms of effective operators can lead to erroneous conclusions. It can overestimate the WIMP signal because of enhancements proportional to $\sqrt{s} / M_{*}$. Or it can underestimate the signal when the mediator can be produced directly and can give a much better collider signal than the "model-independent" WIMP production. The EFT leads to LHC bounds that seem very competitive, but are often only illusory [211].

- Authors of [144] wrote "A direct detection discovery that is in apparent conflict with monojet limits will thus point to a new light state coupling the standard model to the dark sector." Next, almost the same is found in [145]: If a direct DM search experiment were to observe a positive signal, the collider constraints would immediately imply a break down of the effective field theory at collider energies, revealing the existence of a light mediator particle. From these statements one can conclude that the collider constraints are useless or ever irrelevant for the direct DM search experiments. They help nothing. They are unable to give advice where one should better look for a DM signal.

- The simplified models are an extra proof of the inconsistence of the EFT. A typical example of development in this direction is a special White Paper [354], where one discussed a proposal for the consistent interpretation of DM searches at colliders and in direct detection experiments based on the Minimal Simplified Dark Matter (MSDM) model [355]. Nevertheless, the MSDM also has a lot of variants and has the same level of eclecticism as the EFT. It represents only a potential starting point for going beyond the EFT, further additions to the MSDM model, as well as the consideration of alternative approaches, will be required to develop a general strategy for comparing collider and direct DM experiments in the future. 
Next, even these simplified models can be overly simple from the point of view of the true DM physics. Various WIMP-SM interactions are very commonly dictated by interactions with nonhadronic particles. For example, a SUSY neutralino with the correct relic density generically annihilates preferentially into weak vector bosons, but scatters in direct detection primarily through the Higgs boson. All of the approaches above, even if extended to consider interactions with either gauge or Higgs bosons, implicitly assume that for all relevant processes the WIMP interacts dominantly with the same SM field. Ultimately, when a complete model is under consideration, fully focused theoretical vision is the best [23].

Concluding this subsection one can point out that using the model-independent EFT approach for interpretation of the LHC DM search results permanently requires bothering whether it is still valid or already not. To some extent the point is still true for the simplified models.

The $E_{\mathrm{T}}^{\mathrm{miss}}$ is the only measured quantity directly connected with invisible particles. Without further model-dependent assumptions it is impossible to make any statement about the nature of the missing particles [357].

Perhaps, it would be clever to go back to the well-defined (but still too complicated) SUSY framework. In this case one can severely constrain parameter space not of some set of ad hoc effective operators but of one of very promising SUSY models (MSSM, mSUGRA, etc). Experimental rejection, for example, of the MSSM could be a very important result.

This is the first hint to go back to the SUSY.

\subsection{It is not possible to find the DM particle with the LHC or any other accel-} erator. An observation of BSM-excess with the collider $E_{\mathrm{T}}^{\text {miss }}$-signature is very welcome and very important. Nevertheless, one is unable to "directly" discover true DM particles with LHC simply because DM candidates fly away from a detector without any kind of interaction. One can judge them only "inderectly" by the missing mass, missing energy and/or missing momentum in an event. If one would be able to simultaneously measure missing energy and missing 3 -momentum, one would be able to reconstruct the mass of the DM candidate. But even in this case there is no guarantee that this "indirectly detected" particle is the true DM particle - the stable relic particle with clear galactic properties (section 1).

One can stress again that an observation of a DM-like signal (via a collider $E_{\mathrm{T}}^{\text {miss }}$-signature) only proves the production of particle(s) with lifetime $\geq l / c$, where $l$ is the scale of a typical detector. The particles can be the true DM particles only if one can justify an extrapolation from $\simeq 100 \mathrm{~ns}$ up to the lifetime of the Universe (24 orders of magnitude) [22, 61, 13, but this (undoable) justification is not yet enough to prove the DM nature of the particle.

From the collider events with large $E_{\mathrm{T}}^{\text {miss }}$, in the case of non-observation of any BSM-signal, one can only set limits on the masses and couplings of a number of different WIMP-like particles that either depart from a detector tracelessly or fail to decay into detectable particles in the detector. The true DM particles constitute only a subset of this amount of undetected particles, and it is impossible to find model-independent answer to the question of how this subset is large.

In many theories beyond the SM (like SUSY) there are two (or more) massive weakly interacting neutral particles, which are also potential candidates for collider DM particles, because these next-to-lightest particles also can undergo invisible decays and can produce $E_{\mathrm{T}}^{\mathrm{miss}}$-signature. Limits on such signatures tend to be stronger than bounds from direct collider searches for the 
DM itself [127]. There is also a multi-component DM scenario, when a model may contain two (or more) different genuine DM particles, whose production in various combinations will inevitably lead at times to asymmetric event topologies [357].

There is a "hidden problem" with model-independence in the DM search. The results of any model-independent search must afterwards be implemented into one of the modern BSM models of particle physics, for example, supersymmetry. If one day the DM particles is found "in a model-independent way", one inevitably meets a problem to find a proper place for these particles in a new (or still SUSY) model. Today the SUSY looks the best candidate for a model beyond the SM, it unifies all scales, interactions and energies (from extremely high to extremely low), it provides excellent DM particle candidate and so on. Instead of SUSY one can consider any adequate model of new physics.

The only way to "detect DM particle with the LHC" can be realized with simultaneous experimental approval of a complete new physics model, such as SUSY, with all particle masses and couplings coherently described by a set of common parameters. In this case the "indirectly detected" lightest SUSY particle (LSP) will be the very wanted DM candidate (with proper galactic features, relic density, etc).

There is no contradiction to look for the DM particles at the LHC within some realization of the SUSY model right from the very beginning. Often, a more specific model, like MSSM, makes sharper predictions at the cost of generality, but this cost allows verification, or ever complete rejection of the model. Any true DM particle discovery with the LHC is possible only together with the discovery of the SUSY. This is the second hint to go back to the SUSY.

8.3. Still SUSY with RUN-II. There is a common belief that the particle physics accelerator experiments can create and study DM particles in the laboratory. SUSY models with R-parity conservation provide the stable WIMP in the form of the LSP (Lightest SUSY particle). WIMPs are usually the final product of cascade decays of heavier unstable SUSY particles accompanied by SM particles, in particular, with high transverse momentum $p_{\mathrm{T}}$. Therefore one can observe $E_{\mathrm{T}}^{\text {miss }}$ generated by escaped WIMP pairs only if the WIMP pair is tagged by a detectable SM particle yield (jet or photon from initial- or final state radiation) with right the same $E_{\mathrm{T}}^{\mathrm{miss}}$. Furthermore, other LHC measurements can be used to constrain the underlying SUSY model and hence to extract information about the nature of DM, provided the LSP is a very good DM candidate.

The only way to prove the existence of the DM particles at a collider is to prove it together with the SUSY. Unfortunately, this is a very complicated task. First, one should observe BSMexcesses in as many collider observables as possible; second, one should describe these excesses coherently with one set of the SUSY parameters. If the set of parameters gives correct DM density, the LSP can be considered as a true DM particle.

A good example how the LHC helps to learn (within MSSM) something about DM, in particular the relic density, is given in [126]. The relation of the LHC measurements to DM could have several steps. The 1st step is to look for deviations from the SM, for example, in the multi-jet $+E_{\mathrm{T}}^{\mathrm{miss}}$ signatures. If the deviations are observed, the 2nd step is to clarify the SUSY nature of them and to establish the SUSY mass scale using relevant inclusive variables. The 3rd step includes determination of model parameters, selection of particular decay chains and use kinematics to determine mass combinations. Due to the escaping LSPs only the kinematic 
endpoints in the invariant mass distributions of visible decay products can be used to estimate undetected particle masses and to derive the SUSY mass spectrum. As a result, one can obtain the MSSM parameters and the unification scale.

At the end of this step one can perform (model-dependent) estimation of the relic density, which should have a correct value to allow the LSP to claim to be the cold DM candidate. Furthermore, to prove the DM nature of the LSP one inevitably needs extra information (from direct and indirect experiments) concerning the LSP lifetime and the LSP fraction in the observed astrophysical DM [126]. The LHC observed WIMP DM candidate could be only a part of the astrophysical DM [13].

The R-parity violation SUSY models can also contribute to the DM problem, but in the models the WIMP DM candidate can be produced not only by pairs but also in single mode, and should still have a lifetime (against decay into SM particles) compared with that of the Universe. The prospects for the R-parity violating DM search with accelerators are not yet well discussed, perhaps due to their complication and unclearness.

The situation concerning possible discovery of the SUSY (together with the DM) looks much more promising for the LHC RUN-II (13-14 TeV), due to "a bit large" mass of the Higgs boson [142. In the SM the Higgs boson with mass $125-126 \mathrm{GeV}$ makes the vacuum almost unstable and provides very significant indication of New Physics. The most promising candidate for New Physics is the SUSY [142]. This value of the Higgs mass requires a large loop correction and a high TeV-size SUSY scale. The scale can explain suppression of any SUSY contribution to FCNC (flavor-changing neutral current) processes and non-observation of (heavy strong interacting) sparticles in 7-8 TeV data. It also allows for light-mass uncolored sleptons and gauginos (gluino, charginos, neutralinos).

Hence, contrary to RUN-I ( $8 \mathrm{TeV}$ ) RUN-II has a much better chance of observing these lowmass sparticles, in particular the LSP, the best SUSY DM candidate. Furthermore, it was shown that future direct DM experiments such as XENON1T will also be able to explore a large part of the SUSY parameter space consistent with the measured Higgs boson mass [142].

Therefore, it looks today promising, following Pran Nath's paper [142, to perform at the 13-TeV LHC a comprehensive search for the SUSY, having in mind simultaneous search for the SUSY-DM candidate(s). According to John Ellis [358], one should also strongly think about SUSY discovery, which "may not be far away".

The discovery of a light Higgs boson at the LHC opens a broad program of studies and measurements to understand the role of this particle in connection with New Physics and Cosmology. SUSY is the best motivated and most thoroughly formulated and investigated model of New Physics which predicts a light Higgs boson and can solve the DM problem [359].

This is the third hint to go back into the SUSY framework.

8.4. The decisive role of direct DM detection technique. With the unique features of the LHC a new collider-DM-search community has appeared and has very quickly matured. The community has produced a number of experimental LHC-based DM search papers, accompanied with a huge amount of theoretical papers on the subject (see, for example, some of recent papers [207, 355, 360, 361, 362, 363, 364, 365, 366, 367, 368, 369, 370, 371, 372, 373, 374, 375, 376, 377]). Almost every day a paper with a new collider-related DM model is issued (see, for example, [319, 
378, 322, 379, 380]). It looks like the old-fashioned direct-DM-detection community, together with its traditions, achievements and main results, is shifted aside from the main stream on the way to solution of the DM problem. Some precaution here is in order.

First of all one should decide whether an experimental research is aimed at real detection of a DM particle or its goal is to constrain the parameter space of some SUSY, BSM, EFT, simplified or any other model. These very different goals need very different strategies and will have very different results. This point should be clearly understood right from the very beginning.

The above-mentioned discussion shows that with a collider one is unable to detect a true DM particle. One can only constraint the relevant parameter space either with the $E_{\mathrm{T}}^{\text {miss }}$-signature, or with the endpoint or any other technique. Therefore, a collider DM search program inevitably can produce today only exclusion curves as a final result. In the case of an observation of a BSM signal, the curve can only be sharper and more decisive.

Due to a common belief in non-yet-observation of the DM signal (DAMA/LIBRA is ignored), all different DM search techniques (section 2 compete with each other only in the space of the exclusion curves. The goal of this competition has little sense, because it only shows who is today better in looking for nothing (who is a better excluder).

A main physical reason to improve an exclusion curve is usually an attempt to constrain a SUSY-like or BSM model. From non-observation of the BSM signal one can push forward a business on reduction of the parameter space of the models. But it is not very promising and effective, the manifold and flexibility of these models are too large. Every day a new model appears.

The famous model-independent EFT approach together with its modifications do not helps here, they make sense only for comparison of exclusion curves from different search techniques. New forms for a more sophisticated exclusion curve also appear [381], but they still not help a lot with true DM detection, the goal is still competition and consistence between different DM searches.

At the present and foreseeable level of experimental accuracy, simple fighting for the best exclusion curve is almost useless either for real DM detection or for substantial restrictions for SUSY. One should inevitably go beyond the exclusion curve paradigm and aim at registration of the DM particles. As was discussed above, this can only be done together with a discovery of a SUSY-like model.

Furthermore, the key message here concerns the point that only direct DM observation can prove the existence of DM particles. Only in a direct DM experiment one can have a chance to see the galactic nature of the true DM particle population, via measuring the annual modulation of the recoil signal (section 3). This signature is inevitable to prove DM existence. There is no way from the key role of direct DM detection. The other DM search approaches - indirect, collider, astrophysical - can only help the direct DM detection, for example, with mass region search advice, local relic density estimates, etc.

New generations of DM experiments right from their beginning should aim at detection of the DM particles. This will require development of new setups, which will be able to register positive signatures of the DM particle interactions with nuclear targets.

One should try to obtain a reliable recoil energy spectrum. First, very accurate off-line investigation of the measured spectrum allows one to single out different non-WIMP background 
sources and to perform controllable background subtractions. Second, the spectrum allows one to look for the annual modulation effect, the only currently available positive signature of DM particle interactions with terrestrial nuclei.

This effect is not simply a possibility (among many others) of rejecting background, but it is a unique signature which reflects the inner physical properties of the DM interaction with matter. It is a very decisive and eagerly welcomed feature, which is inevitable for the laboratory proof of the existence of the DM population nearby the Earth [104, 114]. Furthermore, natural (solar, supernovae, geo-) neutrino background sooner or latter will be irreducible for direct DM search technique [115], and only a positive signature (like annual modulation, or directional correlations) will be able to eliminate it.

\section{Conclusion}

One is unable to prove, especially model-independently, a discovery of a dark matter particle with an accelerator. What one can do with an accelerator is only to discover evidence for existence of a weakly interacting (massive) particle (WIMP), which could be, or could not be a dark matter (DM) candidate. The WIMP is not yet a true DM particle. A true DM particle possesses a galactic signature, which one should clearly demonstrate. This signature - annual modulation of a signal - is nowadays accessible only for direct DM detection experiments. Therefore, to prove the DM nature of a collider-discovered candidate one must observe it together with this galactic signature in a direct DM search experiment. Colliders can only play here a role of a goal-pointer. Furthermore, being observed sooner or later, the DM particle must be implemented into a modern theoretical framework, like, for example, the Standard Model today, or the supersymmetry (SUSY) in the future.

With this point in mind (together with high complexity of the DM problem), a better strategy may be to look for the DM particles openly relying on a SUSY model. In this model-dependent case determination of the DM properties will be a simple by-product of the SUSY model experimental observation.

The current LHC DM search program uses only the missing transverse energy signature for the WIMP search in the data. Large- $E_{\mathrm{T}}^{\text {miss }}$ search over any possible final state with ATLAS has its own importance for the SUSY or any other beyond-SM theories.

Non-observation of any excess above SM expectations forces the LHC experiments to enter into the same fighting for the best exclusion curve, in which almost all direct DM experiment took place. On this way the model-independent effective field theory approach is commonly used. But this fighting has nothing to do with the real goal to discover a DM particle, especially with an accelerator.

Obviously, if one fails to find a DM-SUSY candidate (together with a SUSY) at the LHC, one must think about another very new BSM framework (or a new collider). It is on the other hand absolutely clear that the SUSY, although in contrast to others being preferred, is not the only candidate for the origin of DM, and other scenarios have also to be investigated.

Perhaps, some words as a non-physical conclusion are in order. Every day at least one new paper with a new model concerning the DM subject can be found in the arXiv. Obviously, this fact reflects the highest level of interest in the DM problem, and one should not stop (or ever strongly reduce) issuing such kind of analysis and papers. A lot of very useful achievements 
are connected with these works - detector, hardware, middleware, software development and study; PhD and PostDoc production and defenses; outreach, spin-off and by-products; society and people education, common scientific community, good international relations and future society organization, etc. Nevertheless, as a precaution, one should not forget with the abovementioned papers and achievements about the main fundamental scientific goal - discovery of galactic dark matter particles.

\section{REFERENCES}

[1] F. Zwicky, The redshift of extragalactic nebulae, Helv. Phys. Acta 6 (1933) 110-127.

[2] M. Livio and J. Silk, Broaden the search for dark matter, Nature 507 (2014) 29, arXiv:1404.2591.

[3] M. Drees and G. Gerbier, Mini-Review of Dark Matter: 2012, arXiv:1204.2373.

[4] T. Saab, An Introduction to Dark Matter Direct Detection Searches and Techniques, arXiv:1203.2566.

[5] G. Bertone, D. Hooper, and J. Silk, Particle dark matter: Evidence, candidates and constraints, Phys. Rept. 405 (2005) 279-390, hep-ph/0404175.

[6] B. Famaey, Dark Matter in the Milky Way, arXiv:1501.0178

[7] F. Iocco, M. Pato, and G. Bertone, Evidence for dark matter in the inner Milky Way, arXiv:1502.0382

[8] R. Durazo, X. Hernandez, and S. Mendoza, Evidence for dark matter in the inner Milky Way...Really?, arXiv: 1503.0750

[9] S. McGaugh, F. Lelli, M. Pawlowski, G. Angus, O. Bienaym, et al., Comment on "Evidence for dark matter in the inner Milky Way", arXiv:1503.0781

[10] F. Iocco, M. Pato, and G. Bertone, Reply to Comment on "Evidence for dark matter in the inner Milky Way", arXiv: 1503.0878

[11] Y. Sofue, Dark Halos of M31 and the Milky Way, arXiv:1504.0536

[12] M. Kuhlen, M. Vogelsberger, and R. Angulo, Numerical Simulations of the Dark Universe: State of the Art and the Next Decade, Phys.Dark Univ. 1 (2012) 50-93, arXiv:1209.5745.

[13] G. B. Gelmini, TASI 2014 Lectures: The Hunt for Dark Matter, arXiv:1502.0132

[14] B. Hoeneisen, Trying to understand dark matter, arXiv:1502.0737

[15] J.-M. Frre, Dark matter variations, arXiv:1504.0822

[16] M. Kamionkowski and A. Kinkhabwala, Galactic halo models and particle dark matter detection, Phys. Rev. D57 (1998) 3256-3263, hep-ph/9710337.

[17] M. Pato, F. Iocco, and G. Bertone, Dynamical constraints on the dark matter distribution in the Milky Way, arXiv: 1504.0632

[18] E. W. Kolb and M. S. Turner, The Early Universe, Front.Phys. 69 (1990) 1-547.

[19] J. L. Feng, Dark Matter Candidates from Particle Physics and Methods of Detection, Ann.Rev.Astron.Astrophys. 48 (2010) 495-545, arXiv:1003.0904.

[20] N. D. Christensen, T. Han, J. Song, and Stefanus, Determining the Dark Matter Particle Mass through Antler Topology Processes at Lepton Colliders, Phys.Rev. D90 (2014) 114029, arXiv:1404.6258.

[21] R. Cerulli, R. Bernabei, P. Belli, F. Cappella, C. Dai, et al., Technical aspects in dark matter investigations, arXiv: 1201.4582

[22] D. Bauer, J. Buckley, M. Cahill-Rowley, R. Cotta, A. Drlica-Wagner, et al., Dark Matter in the Coming Decade: Complementary Paths to Discovery and Beyond, arXiv:1305.1605

[23] A. Askew, S. Chauhan, B. Penning, W. Shepherd, and M. Tripathi, Searching for Dark Matter at Hadron Colliders, Int.J.Mod.Phys. A29 (2014) 1430041, arXiv:1406.5662.

[24] VERITAS Collaboration, B. Zitzer, The VERITAS Dark Matter Program, arXiv:1503.0074

[25] A. Ibarra, A. S. Lamperstorfer, S. L. Gehler, M. Pato, and G. Bertone, On the sensitivity of CTA to gamma-ray boxes from multi-TeV dark matter, arXiv: 1503.0679

[26] Fermi-LAT Collaboration, M. Ackermann et al., Searching for Dark Matter Annihilation from Milky Way Dwarf Spheroidal Galaxies with Six Years of Fermi-LAT Data, arXiv:1503.0264

[27] J. Conrad, J. Cohen-Tanugi, and L. E. Strigari, WIMP searches with gamma rays in the Fermi era: challenges, methods and results, arXiv:1503.0634

[28] Super-Kamiokande Collaboration, K. Choi et al., Search for neutrinos from annihilation of captured low-mass dark matter particles in the Sun by Super-Kamiokande, arXiv:1503.0485

[29] S. Kasuya, M. Kawasaki, and T. T. Yanagida, IceCube potential for detecting the Q-ball dark matter in gauge mediation, arXiv:1502.0071

[30] ANTARES Collaboration, J. Zornoza and G. Lambard, Results and prospects of dark matter searches with ANTARES, Nucl.Instrum.Meth. A742 (2014) 173-176, arXiv:1404.0148.

[31] Baikal Collaboration Collaboration, A. Avrorin et al., Search for neutrino emission from relic dark matter in the Sun with the Baikal NT200 detector, arXiv:1405.3551

[32] D. Hooper and W. Xue, Possibility of Testing the Light Dark Matter Hypothesis with the Alpha Magnetic Spectrometer, Phys.Rev.Lett. 110 (2013), no. 4 041302, arXiv:1210.1220.

[33] PAMELA Collaboration, O. Adriani et al., An anomalous positron abundance in cosmic rays with energies 1.5-100 GeV, Nature 458 (2009) 607-609, arXiv:0810.4995. 
[34] G. Giesen, M. Boudaud, Y. Genolini, V. Poulin, M. Cirelli, et al., AMS-02 antiprotons, at last! Secondary astrophysical component and immediate implications for Dark Matter, arXiv:1504.0427

[35] C. Evoli, D. Gaggero, and D. Grasso, Secondary antiprotons as a Galactic Dark Matter probe, arXiv:1504.0517

[36] S.-J. Lin, X.-J. Bi, P.-F. Yin, and Z.-H. Yu, Implications for dark matter annihilation from the AMS-02 $\bar{p} / p$ ratio, arXiv: 1504.0723

[37] K. Hamaguchi, T. Moroi, and K. Nakayama, AMS-02 Antiprotons from Annihilating or Decaying Dark Matter, arXiv: 1504.0593

[38] C.-H. Chen, C.-W. Chiang, and T. Nomura, Dark matter for excess of AMS-02 positrons and antiprotons, arXiv: 1504.0784

[39] A. Abdo, M. Ackermann, M. Ajello, W. Atwood, L. Baldini, et al., Fermi LAT Search for Photon Lines from 30 to $200 \mathrm{GeV}$ and Dark Matter Implications, Phys.Rev.Lett. 104 (2010) 091302, arXiv:1001.4836.

[40] Fermi-LAT Collaboration, M. Ackermann et al., Fermi LAT Search for Dark Matter in Gamma-ray Lines and the Inclusive Photon Spectrum, Phys.Rev. D86 (2012) 022002, arXiv:1205.2739.

[41] F. Calore, I. Cholis, C. McCabe, and C. Weniger, A Tale of Tails: Dark Matter Interpretations of the Fermi GeV Excess in Light of Background Model Systematics, Phys.Rev. D91 (2015), no. 6 063003, arXiv:1411.4647.

[42] HESS Collaboration Collaboration, A. Abramowski et al., Search for Photon-Linelike Signatures from Dark Matter Annihilations with H.E.S.S., Phys.Rev.Lett. 110 (2013) 041301, arXiv:1301.1173.

[43] K. K. Boddy and J. Kumar, Indirect Detection of Dark Matter Using MeV-Range Gamma-Ray Telescopes, arXiv:1504.0402

[44] K. Ghorbani and H. Ghorbani, Scalar Split WIMPs and Galactic Gamma-Ray Excess, arXiv:1501.0020

[45] K. Ghorbani, Fermionic dark matter with pseudo-scalar Yukawa interaction, JCAP 1501 (2015) 015, arXiv: 1408.4929

[46] P. Cumani, A. Galper, V. Bonvicini, N. Topchiev, O. Adriani, et al., The GAMMA-400 Space Mission, arXiv:1502.0297

[47] B. Bhattacherjee, M. Ibe, K. Ichikawa, S. Matsumoto, and K. Nishiyama, Wino Dark Matter and Future dSph Observations, JHEP 1407 (2014) 080, arXiv:1405.4914.

[48] S. S. Campbell and J. F. Beacom, Combined Flux and Anisotropy Searches Improve Sensitivity to Gamma Rays from Dark Matter, arXiv:1312.3945

[49] L. Bergstrom, The 130 GeV Fingerprint of Right-Handed Neutrino Dark Matter, Phys.Rev. D86 (2012) 103514, arXiv: 1208.6082.

[50] T. Bringmann and C. Weniger, Gamma Ray Signals from Dark Matter: Concepts, Status and Prospects, Phys.Dark Univ. 1 (2012) 194-217, arXiv:1208.5481.

[51] M. Regis, J.-Q. Xia, A. Cuoco, E. Branchini, N. Fornengo, et al., Particle dark matter searches outside the Local neighborhood, arXiv: 1503.0592

[52] M. Y. Khlopov, Introduction to the special issue of Modern Physics Letters A "Indirect dark matter searches", Mod.Phys.Lett. A29 (2014) 1402001, arXiv:1411.2150.

[53] M. A. Barstow, S. Casewell, S. Catalan, C. Copperwheat, B. Gaensicke, et al., White paper: Gaia and the end states of stellar evolution, arXiv: 1407.6163 .

[54] R. Feldmann and D. Spolyar, Detecting Dark Matter Substructures around the Milky Way with Gaia, Mon.Not.Roy.Astron.Soc. 446 (2015) 1000-1012, arXiv:1310.2243.

[55] M. Angeles Perez-Garcia and J. Silk, Constraining decaying dark matter with neutron stars, Phys.Lett. B744 (2015) $13-17$, arXiv: 1403.6111 .

[56] J. Fuller and C. Ott, Dark Matter-induced Collapse of Neutron Stars: A Possible Link Between Fast Radio Bursts and the Missing Pulsar Problem, Mon.Not.Roy.Astron.Soc. L71 (2015) L75, arXiv:1412.6119.

[57] P. W. Graham, S. Rajendran, K. Van Tilburg, and T. D. Wiser, Towards a Bullet-proof test for indirect signals of dark matter, arXiv: 1502.0382

[58] E. Carlson and S. Profumo, When Dark Matter interacts with Cosmic Rays or Interstellar Matter: A Morphological Study, arXiv: 1504.0478

[59] K. R. Dienes, J. Kumar, B. Thomas, and D. Yaylali, Dark-Matter Decay as a Complementary Probe of Multicomponent Dark Sectors, Phys.Rev.Lett. 114 (2015), no. 5 051301, arXiv:1406.4868.

[60] ACT Collaboration, M. Madhavacheril et al., Evidence of Lensing of the Cosmic Microwave Background by Dark Matter Halos, Phys.Rev.Lett. 114 (2015), no. 15 151302, arXiv:1411.7999.

[61] P. Cushman, C. Galbiati, D. McKinsey, H. Robertson, T. Tait, et al., Working Group Report: WIMP Dark Matter Direct Detection, arXiv: 1310.8327

[62] M. W. Goodman and E. Witten, Detectability of certain dark-matter candidates, Phys. Rev. D31 (1985) 3059.

[63] G. Jungman, M. Kamionkowski, and K. Griest, Supersymmetric dark matter, Phys. Rept. 267 (1996) 195-373, hep-ph/9506380.

[64] J. D. Lewin and P. F. Smith, Review of mathematics, numerical factors, and corrections for dark matter experiments based on elastic nuclear recoil, Astropart. Phys. 6 (1996) 87-112.

[65] V. A. Bednyakov, On possible lower bounds for the direct detection rate of susy dark matter, Phys. Atom. Nucl. 66 (2003) 490-493, hep-ph/0201046.

[66] V. A. Bednyakov and H. V. Klapdor-Kleingrothaus, About direct dark matter detection in next-to-minimal supersymmetric standard model, Phys. Rev. D59 (1999) 023514, hep-ph/9802344.

[67] V. A. Bednyakov, S. G. Kovalenko, H. V. Klapdor-Kleingrothaus, and Y. Ramachers, Is susy accessible by direct dark matter detection?, Z. Phys. A357 (1997) 339-347, hep-ph/9606261.

[68] M. Schumann, Dual-Phase Liquid Xenon Detectors for Dark Matter Searches, JINST 9 (2014) C08004, arXiv: 1405.7600 . 
[69] M. Schumann, Dark Matter 2014, Unpublished, (2015) arXiv:1501.0120.

[70] XENON100 Collaboration Collaboration, E. Aprile et al., Dark Matter Results from 225 Live Days of XENON100 Data, Phys.Rev.Lett. 109 (2012) 181301, arXiv:1207.5988.

[71] XENON100 Collaboration Collaboration, E. Aprile et al., Limits on spin-dependent WIMP-nucleon cross sections from 225 live days of XENON100 data, Phys.Rev.Lett. 111 (2013), no. 2 021301, arXiv:1301.6620.

[72] XENON Collaboration, S. Orrigo, Direct Dark Matter Search with XENON100, arXiv:1501.0349

[73] LUX Collaboration Collaboration, D. Akerib et al., First results from the LUX dark matter experiment at the Sanford Underground Research Facility, Phys.Rev.Lett. 112 (2014) 091303, arXiv:1310.8214.

[74] C. Savage, A. Scaffidi, M. White, and A. G. Williams, LUX likelihood and limits on spin-independent and spin-dependent WIMP couplings with LUXCalc, arXiv:1502.0266.

[75] PandaX Collaboration, X. Cao et al., PandaX: A Liquid Xenon Dark Matter Experiment at CJPL, Sci.China Phys.Mech.Astron. 57 (2014) 1476-1494, arXiv:1405.2882.

[76] PandaX Collaboration, M. Xiao et al., First dark matter search results from the PandaX-I experiment, Sci.China Phys.Mech.Astron. 57 (2014) 2024-2030, arXiv:1408.5114.

[77] XENON1T Collaboration, E. Aprile, The XENON1T Dark Matter Search Experiment, Springer Proc.Phys. C12-02-22 (2013) 93-96, arXiv:1206.6288.

[78] XENON Collaboration, E. Aprile et al., Lowering the radioactivity of the photomultiplier tubes for the XENON1T dark matter experiment, arXiv:1503.0769

[79] G. Angloher, M. Bauer, I. Bavykina, A. Bento, C. Bucci, et al., Results from $730 \mathrm{~kg}$ days of the CRESST-II Dark Matter Search, Eur.Phys.J. C72 (2012) 1971, arXiv:1109.0702.

[80] CRESST Collaboration, G. Angloher et al., Probing low WIMP masses with the next generation of CRESST detector, arXiv: 1503.0806

[81] CDMS Collaboration Collaboration, R. Agnese et al., Silicon Detector Dark Matter Results from the Final Exposure of CDMS II, Phys.Rev.Lett. 111 (2013), no. 25 251301, arXiv:1304.4279.

[82] SuperCDMS Collaboration Collaboration, R. Agnese et al., Search for Low-Mass Weakly Interacting Massive Particles with SuperCDMS, Phys.Rev.Lett. 112 (2014), no. 24 241302, arXiv:1402.7137.

[83] R. Agnese, A. Anderson, M. Asai, D. Balakishiyeva, D. Barker, et al., Improved WIMP-search reach of the CDMS II germanium data, arXiv: 1504.0587

[84] C. Aalseth, P. Barbeau, J. Colaresi, J. D. Leon, J. Fast, et al., Maximum Likelihood Signal Extraction Method Applied to 3.4 years of CoGeNT Data, arXiv:1401.6234.

[85] DAMA Collaboration Collaboration, R. Bernabei et al., First results from DAMA/LIBRA and the combined results with DAMA/NaI, Eur.Phys.J. C56 (2008) 333-355, arXiv:0804.2741.

[86] DarkSide Collaboration, D. D'Angelo, DarkSide50 results from first argon run, arXiv:1501.0354

[87] PICASSO Collaboration Collaboration, S. Archambault et al., Constraints on Low-Mass WIMP Interactions on ${ }^{19}$ F from PICASSO, Phys.Lett. B711 (2012) 153-161, arXiv:1202.1240.

[88] Super-Kamiokande Collaboration Collaboration, S. Desai et al., Search for dark matter WIMPs using upward through-going muons in Super-Kamiokande, Phys.Rev. D70 (2004) 083523, hep-ex/0404025.

[89] ICECUBE Collaboration Collaboration, R. Abbasi et al., Limits on a muon flux from neutralino annihilations in the Sun with the IceCube 22-string detector, Phys.Rev.Lett. 102 (2009) 201302, arXiv:0902.2460].

[90] E. Behnke, J. Behnke, S. Brice, D. Broemmelsiek, J. Collar, et al., Improved Limits on Spin-Dependent WIMP-Proton Interactions from a Two Liter CF $C_{3}$ B Bubble Chamber, Phys.Rev.Lett. 106 (2011) 021303, arXiv: 1008.3518.

[91] M. Felizardo, T. Girard, T. Morlat, A. Fernandes, A. Ramos, et al., Final Analysis and Results of the Phase II SIMPLE Dark Matter Search, Phys.Rev.Lett. 108 (2012) 201302, arXiv:1106.3014.

[92] X.-J. Bi, P.-F. Yin, and Q. Yuan, Status of Dark Matter Detection, Front.Phys.China 8 (2013) 794-827, arXiv: 1409.4590 .

[93] J. Engel, S. Pittel, and P. Vogel, Nuclear physics of dark matter detection, Int.J.Mod.Phys. E1 (1992) 1-37.

[94] M. T. Ressell, M. B. Aufderheide, S. D. Bloom, K. Griest, G. J. Mathews, et al., Nuclear shell model calculations of neutralino - nucleus cross-sections for Si-29 and Ge-73, Phys.Rev. D48 (1993) 5519-5535.

[95] R. Bernabei et al., Dark matter search, Riv. Nuovo Cim. 26 (2003) 1-73, astro-ph/0307403.

[96] M. Ressell and D. Dean, Spin dependent neutralino - nucleus scattering for A approximately 127 nuclei, Phys. Rev. C56 (1997) 535-546, hep-ph/9702290.

[97] V. Bednyakov and F. Simkovic, Nuclear spin structure in dark matter search: The Zero momentum transfer limit, Phys.Part.Nucl. 36 (2005) 131-152, hep-ph/0406218.

[98] V. Bednyakov and F. Simkovic, Nuclear spin structure in dark matter search: The Finite momentum transfer limit, Phys.Part.Nucl. 37 (2006) S106-S128, hep-ph/0608097.

[99] V. A. Bednyakov and H. V. Klapdor-Kleingrothaus, On dark matter search after dama with ge-73, Phys. Rev. D70 (2004) 096006, hep-ph/0404102.

[100] K. Freese, J. A. Frieman, and A. Gould, Signal modulation in cold dark matter detection, Phys. Rev. D37 (1988) 3388.

[101] SuperCDMS Collaboration, K. Schneck et al., Dark matter effective field theory scattering in direct detection experiments, arXiv:1503.0337

[102] N. Spooner, Direct Dark Matter Searches, J.Phys.Soc.Jap. 76 (2007) 111016, arXiv:0705.3345.

[103] R. Catena and P. Gondolo, Global limits and interference patterns in dark matter direct detection, arXiv:1504.0655

[104] V. Bednyakov and H. Klapdor-Kleingrothaus, Direct Search for Dark Matter — Striking the Balance - and the Future, Phys.Part.Nucl. 40 (2009) 583-611, arXiv:0806.3917. 
[105] K. Freese, M. Lisanti, and C. Savage, Colloquium: Annual modulation of dark matter, Rev.Mod.Phys. 85 (2013) 1561-1581, arXiv:1209.3339.

[106] V. Bednyakov and H. Klapdor-Kleingrothaus, Possibilities of directly detecting dark-matter particles in the next-to-minimal supersymmetric standard model, Phys.Atom.Nucl. 62 (1999) 966-974.

[107] E. Del Nobile, G. B. Gelmini, and S. J. Witte, Target dependence of the annual modulation in direct dark matter searches, arXiv: 1504.0677

[108] P. Belli, R. Bernabei, A. Bottino, F. Cappella, R. Cerulli, et al., Observations of annual modulation in direct detection of relic particles and light neutralinos, Phys.Rev. D84 (2011) 055014, arXiv:1106.4667.

[109] J. D. Vergados, Theoretical directional and modulated rates for direct susy dark matter detection, Phys. Rev. D67 (2003) 103003, hep-ph/0303231.

[110] B. Morgan, A. M. Green, and N. J. C. Spooner, Directional statistics for wimp direct detection, Phys. Rev. D71 (2005) 103507, astro-ph/0408047.

[111] G. Mohlabeng, K. Kong, J. Li, A. Para, and J. Yoo, Dark Matter Directionality Revisited with a High Pressure Xenon Gas Detector, arXiv: 1503.0393

[112] V. A. Bednyakov, H. V. Klapdor-Kleingrothaus, and I. V. Krivosheina, New constraints on spin-dependent WIMP-neutron interactions from HDMS with natural Ge and Ge-73, Phys. Atom. Nucl. 71 (2008) 111-116.

[113] J. D. Vergados, Direct susy dark matter detection: Theoretical rates due to the spin, J. Phys. G30 (2004) 1127-1144, hep-ph/0406134.

[114] V. Bednyakov, One needs positive signatures for detection of Dark Matter, Phys.Part.Nucl. 44 (2013) 220-228, arXiv: 1207.2899.

[115] A. Gtlein, G. Angloher, A. Bento, C. Bucci, L. Canonica, et al., Impact of Coherent Neutrino Nucleus Scattering on Direct Dark Matter Searches based on $\mathrm{CaWO}_{4}$ Crystals, arXiv:1408.2357

[116] R. Bernabei, Dark Matter Particles in the Galactic Halo, Physics 15 (2014) 10, arXiv:1412.6524.

[117] R. Bernabei, P. Belli, F. Cappella, V. Caracciolo, S. Castellano, et al., Final model independent result of DAMA/LIBRA-phase1, Eur.Phys.J. C73 (2013), no. 12 2648, arXiv:1308.5109.

[118] C. Aalseth, P. Barbeau, J. Colaresi, J. Collar, J. Diaz Leon, et al., Search for an Annual Modulation in a P-type Point Contact Germanium Dark Matter Detector, Phys.Rev.Lett. 107 (2011) 141301, arXiv:1106.0650.

[119] CoGeNT Collaboration Collaboration, C. Aalseth et al., CoGeNT: A Search for Low-Mass Dark Matter using p-type Point Contact Germanium Detectors, Phys.Rev. D88 (2013), no. 1 012002, arXiv:1208.5737.

[120] A. Berlin, T. Lin, M. Low, and L.-T. Wang, Neutralinos in Vector Boson Fusion at High Energy Colliders, arXiv: 1502.0504

[121] V. Bednyakov and H. Klapdor-Kleingrothaus, SUSY spectrum constraints on direct dark matter detection, Phys.Rev. D62 (2000) 043524, hep-ph/9908427.

[122] V. Bednyakov, H. Klapdor-Kleingrothaus, and H. Tu, Higgs bosons and the indirect search for WIMPs, Phys.Rev. D64 (2001) 075004, hep-ph/0101223.

[123] V. Bednyakov, Y. Budagov, A. Gladyshev, D. Kazakov, E. Khramov, et al., On the LHC observation of gluinos from the Egret-preferred region, Phys.Atom.Nucl. 72 (2009) 619-637.

[124] ATLAS Collaboration Collaboration, T. Eifert, Searches for supersymmetry at the LHC and its dark matter candidate,

[125] G. Polesello and D. Tovey, Constraining SUSY dark matter with the ATLAS detector at the LHC, JHEP 0405 (2004) 071, hep-ph/0403047.

[126] ATLAS Collaboration, E. Barberio, SUSY searches at LHC and Dark Matter,

[127] A. Ismail, Dark matter complementarity in the phenomenological MSSM, AIP Conf.Proc. 1604 (2014) 53-65.

[128] L. Roszkowski, E. M. Sessolo, and A. J. Williams, Prospects for dark matter searches in the pMSSM, JHEP 1502 (2015) 014, arXiv:1411.5214.

[129] M. E. C. Catalan, S. Ando, C. Weniger, and F. Zandanel, Indirect and direct detection prospect for TeV dark matter in the MSSM-9, arXiv:1503.0059

[130] L. Aparicio, M. Cicoli, B. Dutta, S. Krippendorf, A. Maharana, et al., Non-thermal CMSSM with a 125 GeV Higgs, arXiv: 1502.0567

[131] K. Kowalska, L. Roszkowski, E. M. Sessolo, and A. J. Williams, GUT-inspired SUSY and the muon g-2 anomaly: prospects for $\mathrm{LHC} 14 \mathrm{TeV}$, arXiv:1503.0821

[132] A. Achterberg, S. Caron, L. Hendriks, R. Ruiz de Austri, and C. Weniger, A description of the Galactic Center excess in the Minimal Supersymmetric Standard Model and the Dark Matter signatures for the LHC and direct and indirect detection experiments, arXiv: 1502.0570

[133] C. Han, D. Kim, S. Munir, and M. Park, O(1) GeV dark matter in SUSY and a very light pseudoscalar at the LHC, arXiv: 1504.0508

[134] T. Gherghetta, B. von Harling, A. D. Medina, M. A. Schmidt, and T. Trott, SUSY Implications from WIMP Annihilation into Scalars at the Galactic Centre, arXiv:1502.0717

[135] C. Arina, M. E. C. Catalan, S. Kraml, S. Kulkarni, and U. Laa, Constraints on sneutrino dark matter from LHC Run 1, arXiv:1503.0296

[136] H. An, P. B. Dev, Y. Cai, and R. Mohapatra, Sneutrino Dark Matter in Gauged Inverse Seesaw Models for Neutrinos, Phys.Rev.Lett. 108 (2012) 081806, arXiv:1110.1366.

[137] P. Bhupal Dev, S. Mondal, B. Mukhopadhyaya, and S. Roy, Phenomenology of Light Sneutrino Dark Matter in cMSSM/mSUGRA with Inverse Seesaw, JHEP 1209 (2012) 110, arXiv:1207.6542.

[138] S. Banerjee, P. S. B. Dev, S. Mondal, B. Mukhopadhyaya, and S. Roy, Invisible Higgs Decay in a Supersymmetric Inverse Seesaw Model with Light Sneutrino Dark Matter, JHEP 1310 (2013) 221, arXiv:1306.2143. 
[139] M. Kakizaki, E.-K. Park, J.-h. Park, and A. Santa, Phenomenological constraints on light mixed sneutrino dark matter scenarios, arXiv: 1503.0678

[140] D. Barducci, A. Belyaev, A. K. M. Bharucha, W. Porod, and V. Sanz, Uncovering Natural Supersymmetry via the interplay between the LHC and Direct Dark Matter Detection, arXiv:1504.0247

[141] S. Akula, D. Feldman, Z. Liu, P. Nath, and G. Peim, New Constraints on Dark Matter from CMS and ATLAS Data, Mod.Phys.Lett. A26 (2011) 1521-1535, arXiv:1103.5061.

[142] P. Nath, Supersymmetry after the Higgs, arXiv:1501.0167

[143] J. Goodman, M. Ibe, A. Rajaraman, W. Shepherd, T. M. Tait, et al., Constraints on Light Majorana dark Matter from Colliders, Phys.Lett. B695 (2011) 185-188, arXiv:1005.1286.

[144] Y. Bai, P. J. Fox, and R. Harnik, The Tevatron at the Frontier of Dark Matter Direct Detection, JHEP 1012 (2010) 048, arXiv:1005.3797.

[145] J. Goodman, M. Ibe, A. Rajaraman, W. Shepherd, T. M. Tait, et al., Constraints on Dark Matter from Colliders, Phys.Rev. D82 (2010) 116010, arXiv:1008.1783.

[146] P. J. Fox, R. Harnik, J. Kopp, and Y. Tsai, Missing Energy Signatures of Dark Matter at the LHC, Phys.Rev. D85 (2012) 056011, arXiv:1109.4398.

[147] G. Busoni, Limitation of EFT for DM interactions at the LHC, PoS DIS2014 (2014) 134, arXiv:1411.3600

[148] G. Busoni, A. De Simone, E. Morgante, and A. Riotto, On the Validity of the Effective Field Theory for Dark Matter Searches at the LHC, Phys.Lett. B728 (2014) 412-421, arXiv:1307.2253.

[149] CMS Collaboration Collaboration, S. Lowette, Search for Dark Matter at CMS, arXiv:1410.3762

[150] V. A. Mitsou, Overview of searches for dark matter at the LHC, arXiv:1402.3673

[151] ATLAS Collaboration Collaboration, G. Aad et al., Search for dark matter candidates and large extra dimensions in events with a jet and missing transverse momentum with the ATLAS detector, JHEP 1304 (2013) 075, arXiv:1210.4491.

[152] CDF Collaboration, T. Aaltonen et al., Search for large extra dimensions in final states containing one photon or jet and large missing transverse energy produced in p p collisions at $\sqrt{s}=1.96-$ TeV, Phys.Rev.Lett. 101 (2008) 181602, arXiv:0807.3132.

[153] ATLAS Collaboration Collaboration, G. Aad et al., Search for new phenomena in final states with an energetic jet and large missing transverse momentum in pp collisions at $\sqrt{s}=8 \mathrm{TeV}$ with the ATLAS detector, arXiv: 1502.0151

[154] G. Angloher, S. Cooper, R. Keeling, H. Kraus, J. Marchese, et al., Limits on WIMP dark matter using sapphire cryogenic detectors, Astropart.Phys. 18 (2002) 43-55.

[155] CDMS-II Collaboration Collaboration, Z. Ahmed et al., Dark Matter Search Results from the CDMS II Experiment, Science $\mathbf{3 2 7}$ (2010) 1619-1621, arXiv:0912.3592.

[156] XENON Collaboration Collaboration, J. Angle et al., First Results from the XENON10 Dark Matter Experiment at the Gran Sasso National Laboratory, Phys.Rev.Lett. 100 (2008) 021303, arXiv:0706.0039.

[157] CoGeNT collaboration Collaboration, C. Aalseth et al., Results from a Search for Light-Mass Dark Matter with a P-type Point Contact Germanium Detector, Phys.Rev.Lett. 106 (2011) 131301, arXiv:1002.4703.

[158] XENON100 Collaboration Collaboration, E. Aprile et al., First Dark Matter Results from the XENON100 Experiment, Phys.Rev.Lett. 105 (2010) 131302, arXiv:1005.0380.

[159] D. Akerib, M. Attisha, C. Bailey, L. Baudis, D. A. Bauer, et al., The SuperCDMS proposal for dark matter detection, Nucl.Instrum. Meth. A559 (2006) 411-413.

[160] XENON100 Collaboration Collaboration, E. Aprile and L. Baudis, Status and Sensitivity Projections for the XENON100 Dark Matter Experiment, PoS IDM2008 (2008) 018, arXiv:0902.4253.

[161] S. Archambault, F. Aubin, M. Auger, E. Behnke, B. Beltran, et al., Dark Matter Spin-Dependent Limits for WIMP Interactions on F-19 by PICASSO, Phys.Lett. B682 (2009) 185-192, arXiv:0907.0307.

[162] KIMS Collaboration Collaboration, H. Lee et al., Limits on WIMP-nucleon cross section with CsI(Tl) crystal detectors, Phys.Rev.Lett. 99 (2007) 091301, arXiv:0704.0423.

[163] G. Sciolla, J. Battat, T. Caldwell, D. Dujmic, P. Fisher, et al., The DMTPC project, J.Phys.Conf.Ser. 179 (2009) 012009, arXiv:0903.3895.

[164] J. Gramling, Probing dark matter with monojets in ATLAS at the LHC, PoS Corfu2012 (2013) 058.

[165] ATLAS Collaboration, E. Diehl, The search for dark matter using monojets and monophotons with the ATLAS detector, AIP Conf.Proc. 1604 (2014) 324-330.

[166] ATLAS Collaboration Collaboration, G. Aad et al., Search for dark matter candidates and large extra dimensions in events with a photon and missing transverse momentum in pp collision data at $\sqrt{s}=7$ TeV with the ATLAS detector, Phys.Rev.Lett. 110 (2013) 011802, arXiv:1209.4625.

[167] CMS Collaboration Collaboration, S. Chatrchyan et al., Search for Dark Matter and Large Extra Dimensions in pp Collisions Yielding a Photon and Missing Transverse Energy, Phys.Rev.Lett. 108 (2012) 261803, arXiv: 1204.0821 .

[168] CMS Collaboration, S. Chatrchyan et al., Search for New Physics with a Mono-Jet and Missing Transverse Energy in pp Collisions at $\sqrt{s}=7$ TeV, Phys.Rev.Lett. 107 (2011) 201804, arXiv:1106.4775.

[169] ATLAS Collaboration Collaboration, G. Aad et al., Search for dark matter in events with a hadronically decaying $W$ or $Z$ boson and missing transverse momentum in pp collisions at $\sqrt{s}=8$ TeV with the ATLAS detector, Phys.Rev.Lett. 112 (2014), no. 4 041802, arXiv:1309.4017.

[170] Y. Bai and T. M. Tait, Searches with Mono-Leptons, Phys.Lett. B723 (2013) 384-387, arXiv:1208.4361.

[171] CDMS-II Collaboration Collaboration, Z. Ahmed et al., Results from a Low-Energy Analysis of the CDMS II Germanium Data, Phys.Rev.Lett. 106 (2011) 131302, arXiv:1011.2482. 
[172] IceCube collaboration Collaboration, M. Aartsen et al., Search for dark matter annihilations in the Sun with the 79-string IceCube detector, Phys.Rev.Lett. 110 (2013), no. 13 131302, arXiv:1212.4097.

[173] COUPP Collaboration Collaboration, E. Behnke et al., First Dark Matter Search Results from a 4-kg $\mathrm{CF}_{3} I$ Bubble Chamber Operated in a Deep Underground Site, Phys.Rev. D86 (2012), no. 5 052001, arXiv:1204.3094.

[174] ATLAS Collaboration Collaboration, G. Aad et al., Search for Invisible Decays of a Higgs Boson Produced in Association with a Z Boson in ATLAS, Phys.Rev.Lett. 112 (2014) 201802, arXiv:1402.3244.

[175] LHC Higgs Cross Section Working Group Collaboration, S. Heinemeyer et al., Handbook of LHC Higgs Cross Sections: 3. Higgs Properties, arXiv:1307.1347

[176] S. Kanemura, S. Matsumoto, T. Nabeshima, and N. Okada, Can WIMP Dark Matter overcome the Nightmare Scenario?, Phys.Rev. D82 (2010) 055026, arXiv:1005.5651].

[177] A. Djouadi, O. Lebedev, Y. Mambrini, and J. Quevillon, Implications of LHC searches for Higgs-portal dark matter, Phys.Lett. B709 (2012) 65-69, arXiv:1112.3299.

[178] XENON10 Collaboration Collaboration, J. Angle et al., A search for light dark matter in XENON10 data, Phys.Rev.Lett. 107 (2011) 051301, arXiv:1104.3088.

[179] P. J. Fox, J. Kopp, M. Lisanti, and N. Weiner, A CoGeNT Modulation Analysis, Phys.Rev. D85 (2012) 036008, arXiv:1107.0717.

[180] SuperCDMS Collaboration Collaboration, R. Agnese et al., Search for Low-Mass Weakly Interacting Massive Particles Using Voltage-Assisted Calorimetric Ionization Detection in the SuperCDMS Experiment, Phys.Rev.Lett. 112 (2014), no. 4 041302, arXiv:1309.3259.

[181] ATLAS Collaboration Collaboration, G. Aad et al., Search for dark matter in events with a $Z$ boson and missing transverse momentum in pp collisions at $\sqrt{s}=8 \mathrm{TeV}$ with the ATLAS detector, Phys.Rev. D90 (2014) 012004, arXiv: 1404.0051

[182] N. F. Bell, J. B. Dent, A. J. Galea, T. D. Jacques, L. M. Krauss, et al., Searching for Dark Matter at the LHC with a Mono-Z, Phys.Rev. D86 (2012) 096011, arXiv:1209.0231.

[183] ATLAS Collaboration Collaboration, G. Aad et al., Search for dark matter in events with heavy quarks and missing transverse momentum in pp collisions with the ATLAS detector, arXiv:1410.4031

[184] C. Rogan, Kinematical variables towards new dynamics at the LHC, arXiv:1006.2727

[185] P. Agrawal, B. Batell, D. Hooper, and T. Lin, Flavored Dark Matter and the Galactic Center Gamma-Ray Excess, Phys.Rev. D90 (2014), no. 6 063512, arXiv:1404.1373.

[186] ATLAS Collaboration Collaboration, G. Aad et al., Search for new phenomena in events with a photon and missing transverse momentum in pp collisions at $\sqrt{s}=8 \mathrm{TeV}$ with the ATLAS detector, Phys.Rev. D91 (2015), no. 1 012008, arXiv:1411.1559.

[187] A. Nelson, L. M. Carpenter, R. Cotta, A. Johnstone, and D. Whiteson, Confronting the Fermi Line with LHC data: an Effective Theory of Dark Matter Interaction with Photons, Phys.Rev. D89 (2014), no. 5 056011, arXiv: 1307.5064.

[188] C. Weniger, A Tentative Gamma-Ray Line from Dark Matter Annihilation at the Fermi Large Area Telescope, JCAP 1208 (2012) 007, arXiv:1204.2797.

[189] WMAP Collaboration Collaboration, E. Komatsu et al., Seven-Year Wilkinson Microwave Anisotropy Probe (WMAP) Observations: Cosmological Interpretation, Astrophys.J.Suppl. 192 (2011) 18, arXiv:1001.4538.

[190] ATLAS Collaboration Collaboration, G. Aad et al., Search for new particles in events with one lepton and missing transverse momentum in pp collisions at $\sqrt{s}=8 \mathrm{TeV}$ with the ATLAS detector, JHEP 1409 (2014) 037, arXiv: 1407.7494.

[191] M. Chizhov and G. Dvali, Origin and Phenomenology of Weak-Doublet Spin-1 Bosons, Phys.Lett. B703 (2011) 593-598, arXiv:0908.0924.

[192] M. Chizhov, V. Bednyakov, and J. Budagov, Proposal for chiral bosons search at LHC via their unique new signature, Phys.Atom.Nucl. 71 (2008) 2096-2100, arXiv:0801.4235.

[193] Do Collaboration Collaboration, V. Abazov et al., Search for large extra dimensions via single photon plus missing energy final states at $\sqrt{s}=1.96$-TeV, Phys.Rev.Lett. 101 (2008) 011601, arXiv:0803.2137.

[194] CDF Collaboration Collaboration, T. Aaltonen et al., A Search for dark matter in events with one jet and missing transverse energy in p $\bar{p}$ collisions at $\sqrt{s}=1.96 \mathrm{TeV}$, Phys.Rev.Lett. 108 (2012) 211804, arXiv:1203.0742.

[195] CMS Collaboration, S. Chatrchyan et al., Search for dark matter and large extra dimensions in monojet events in pp collisions at $\sqrt{s}=7 \mathrm{TeV}, \mathrm{JHEP} 1209$ (2012) 094, arXiv:1206.5663.

[196] ATLAS Collaboration, G. Aad et al., Search for new phenomena with the monojet and missing transverse momentum signature using the ATLAS detector in $\sqrt{s}=7$ TeV proton-proton collisions, Phys.Lett. B705 (2011) 294-312, arXiv:1106.5327.

[197] CMS Collaboration, V. Khachatryan et al., Search for dark matter, extra dimensions, and unparticles in monojet events in proton-proton collisions at $\sqrt{s}=8 \mathrm{TeV}$, arXiv:1408.3583.

[198] CMS Collaboration, V. Khachatryan et al., Search for physics beyond the standard model in final states with a lepton and missing transverse energy in proton-proton collisions at $\sqrt{s}=8 \mathrm{TeV}$, arXiv:1408.2745

[199] A. L. Read, Presentation of search results: The CL(s) technique, J.Phys. G28 (2002) 2693-2704.

[200] G. Steigman and M. S. Turner, Cosmological Constraints on the Properties of Weakly Interacting Massive Particles, Nucl.Phys. B253 (1985) 375.

[201] WMAP Collaboration, G. Hinshaw et al., Nine-Year Wilkinson Microwave Anisotropy Probe (WMAP) Observations: Cosmological Parameter Results, Astrophys.J.Suppl. 208 (2013) 19, arXiv:1212.5226.

[202] Fermi-LAT Collaboration Collaboration, M. Ackermann et al., Dark matter constraints from observations of 25 Milky Way satellite galaxies with the Fermi Large Area Telescope, Phys.Rev. D89 (2014) 042001, arXiv:1310.0828. 
[203] HESS Collaboration, A. Abramowski et al., Search for a Dark Matter annihilation signal from the Galactic Center halo with H.E.S.S, Phys.Rev.Lett. 106 (2011) 161301, arXiv:1103.3266.

[204] Planck Collaboration, P. Ade et al., Planck 2013 results. XVI. Cosmological parameters, Astron.Astrophys. 571 (2014) A16, arXiv:1303.5076.

[205] F. Bishara, J. Brod, P. Uttayarat, and J. Zupan, Nonstandard Yukawa Couplings and Higgs Portal Dark Matter, arXiv: 1504.0402

[206] M. Dutra, C. A. d. S. Pires, and P. S. R. da Silva, Majorana Dark Matter in Minimal Higgs Portal Models after $L U X, \operatorname{arXiv:1504.0722}$

[207] M. A. Fedderke, J.-Y. Chen, E. W. Kolb, and L.-T. Wang, The Fermionic Dark Matter Higgs Portal: an effective field theory approach, JHEP 1408 (2014) 122, arXiv: 1404.2283.

[208] CMS Collaboration, S. Chatrchyan et al., Search for invisible decays of Higgs bosons in the vector boson fusion and associated ZH production modes, Eur.Phys.J. C74 (2014) 2980, arXiv:1404.1344.

[209] ATLAS Collaboration, G. Aad et al., Search for invisible decays of the Higgs boson produced in association with a hadronically decaying vector boson in pp collisions at $\sqrt{s}=8 \mathrm{TeV}$ with the ATLAS detector, arXiv:1504.0432

[210] CMS Collaboration, V. Khachatryan et al., Search for the production of dark matter in association with top-quark pairs in the single-lepton final state in proton-proton collisions at sqrt(s) $=8 \mathrm{TeV}$, arXiv:1504.0319

[211] A. De Simone, G. F. Giudice, and A. Strumia, Benchmarks for Dark Matter Searches at the LHC, JHEP 1406 (2014) 081, arXiv:1402.6287.

[212] N. Fernandez, J. Kumar, I. Seong, and P. Stengel, Complementary Constraints on Light Dark Matter from Heavy Quarkonium Decays, Phys.Rev. D90 (2014) 015029, arXiv:1404.6599.

[213] NA48/2 Collaboration Collaboration, E. Goudzovski, Search for the dark photon in $\pi^{0}$ decays by the NA48/2 experiment at CERN, arXiv:1412.8053

[214] CERN NA48/2 Collaboration, Search for the dark photon in $\pi^{0}$ decays, arXiv:1504.0060

[215] BaBar Collaboration Collaboration, J. Lees et al., Search for a Dark Photon in $e^{+} e^{-}$Collisions a BaBar, Phys.Rev.Lett. 113 (2014), no. 20 201801, arXiv:1406.2980.

[216] Muon g-2 Collaboration, G. Bennett et al., Final Report of the Muon E821 Anomalous Magnetic Moment Measurement at BNL, Phys.Rev. D73 (2006) 072003, hep-ex/0602035.

[217] BaBar Collaboration, G. Eigen, Direct Searches for New Physics Particles at BABAR, arXiv:1503.0286

[218] B. Echenard, R. Essig, and Y.-M. Zhong, Projections for Dark Photon Searches at Mu3e, arXiv:1411.1770

[219] N. Arkani-Hamed, D. P. Finkbeiner, T. R. Slatyer, and N. Weiner, A Theory of Dark Matter, Phys.Rev. D79 (2009) 015014, arXiv:0810.0713.

[220] C. Cheung, J. T. Ruderman, L.-T. Wang, and I. Yavin, Lepton Jets in (Supersymmetric) Electroweak Processes, JHEP 1004 (2010) 116, arXiv:0909.0290.

[221] R. Foot and S. Vagnozzi, Dissipative hidden sector dark matter, Phys.Rev. D91 (2015) 023512, arXiv:1409.7174.

[222] R. Foot and S. Vagnozzi, Diurnal modulation signal from dissipative hidden sector dark matter, arXiv:1412.0762

[223] K. Kong, H.-S. Lee, and M. Park, Charged Higgs Probes of Dark Bosons at the LHC, arXiv:1408.4021

[224] K. Kong, H.-S. Lee, and M. Park, Dark decay of the top quark, Phys.Rev. D89 (2014), no. 7 074007, arXiv: 1401.5020 .

[225] H. Davoudiasl, W. J. Marciano, R. Ramos, and M. Sher, Charged Higgs Discovery in the W plus "Dark" Vector Boson Decay Mode, Phys.Rev. D89 (2014), no. 11 115008, arXiv:1401.2164.

[226] A. Gupta, R. Primulando, and P. Saraswat, A New Probe of Dark Sector Dynamics at the LHC, arXiv:1504.0138

[227] Y. Bai, J. Bourbeau, and T. Lin, Dark Matter Searches with a Mono-Z' jet, arXiv:1504.0139

[228] M. Autran, K. Bauer, T. Lin, and D. Whiteson, Mono-Z': searches for dark matter in events with a resonance and missing transverse energy, arXiv: 1504.0138

[229] H.-S. Lee, Muon g-2 Anomaly and Dark Leptonic Gauge Boson, Phys.Rev. D90 (2014) 091702, arXiv:1408.4256

[230] HADES Collaboration, G. Agakishiev et al., Searching a Dark Photon with HADES, Phys.Lett. B731 (2014) 265-271, arXiv:1311.0216.

[231] KLOE-2 Collaboration, D. Babusci et al., Search for light vector boson production in $e^{+} e^{-} \rightarrow \mu^{+} \mu^{-} \gamma$ interactions with the KLOE experiment, Phys.Lett. B736 (2014) 459-464, arXiv:1404.7772.

[232] F. Xu, Dark Z Implication for Flavor Physics, arXiv:1504.0741

[233] B. Batell, R. Essig, and Z. Surujon, Strong Constraints on Sub-GeV Dark Sectors from SLAC Beam Dump E137, Phys.Rev.Lett. 113 (2014), no. 17 171802, arXiv:1406.2698

[234] S. Gninenko, N. Krasnikov, and V. Matveev, The muon g-2 and searches for a new electrophobic sub-GeV dark boson in a missing-energy experiment at CERN, arXiv:1412.1400

[235] D. Gorbunov, A. Makarov, and I. Timiryasov, Decaying light particles in the SHiP experiment: Signal rate estimates for hidden photons, Phys.Rev. D91 (2015), no. 3 035027, arXiv:1411.4007.

[236] S. Alekhin, W. Altmannshofer, T. Asaka, B. Batell, F. Bezrukov, et al., A facility to Search for Hidden Particles at the CERN SPS: the SHiP physics case, arXiv:1504.0485

[237] D. Curtin, R. Essig, S. Gori, and J. Shelton, Illuminating Dark Photons with High-Energy Colliders, JHEP 1502 (2015) 157, arXiv:1412.0018.

[238] E. Izaguirre, G. Krnjaic, P. Schuster, and N. Toro, Testing GeV-Scale Dark Matter with Fixed-Target Missing Momentum Experiments, arXiv:1411.1404

[239] E. Izaguirre, G. Krnjaic, P. Schuster, and N. Toro, New Electron Beam-Dump Experiments to Search for MeV to few-GeV Dark Matter, Phys.Rev. D88 (2013) 114015, arXiv:1307.6554.

[240] P. deNiverville, D. McKeen, and A. Ritz, Signatures of sub-GeV dark matter beams at neutrino experiments, Phys.Rev. D86 (2012) 035022, arXiv:1205.3499. 
[241] M. D. Diamond and P. Schuster, Searching for Light Dark Matter with the SLAC Millicharge Experiment, Phys.Rev.Lett. 111 (2013), no. 22 221803, arXiv:1307.6861.

[242] BDX Collaboration Collaboration, M. Battaglieri et al., Dark matter search in a Beam-Dump eXperiment (BDX) at Jefferson Lab, arXiv: 1406.3028.

[243] J. D. Bjorken, R. Essig, P. Schuster, and N. Toro, New Fixed-Target Experiments to Search for Dark Gauge Forces, Phys.Rev. D80 (2009) 075018, arXiv:0906.0580.

[244] E. Izaguirre, G. Krnjaic, P. Schuster, and N. Toro, Physics Motivation for a Pilot Dark Matter Search at Jefferson Laboratory, Phys.Rev. D90 (2014) 014052, arXiv:1403.6826.

[245] J. Balewski, J. Bernauer, J. Bessuille, R. Corliss, R. Cowan, et al., The DarkLight Experiment: A Precision Search for New Physics at Low Energies, arXiv:1412.4717

[246] H. An, M. Pospelov, J. Pradler, and A. Ritz, Direct Detection Constraints on Dark Photon Dark Matter, arXiv: 1412.8378

[247] B. Dutta, Dark Matter Searches at Accelerator Facilities, arXiv:1403.6217

[248] P. J. Fox and E. Poppitz, Leptophilic Dark Matter, Phys.Rev. D79 (2009) 083528, arXiv:0811.0399.

[249] J. Kopp, V. Niro, T. Schwetz, and J. Zupan, DAMA/LIBRA and leptonically interacting Dark Matter, Phys.Rev. D80 (2009) 083502, arXiv:0907.3159.

[250] A. Freitas and S. Westhoff, Leptophilic Dark Matter in Lepton Interactions at LEP and ILC, JHEP 1410 (2014) 116, arXiv:1408.1959.

[251] H. Dreiner, M. Huck, M. Krmer, D. Schmeier, and J. Tattersall, Illuminating Dark Matter at the ILC, Phys.Rev. D87 (2013), no. 7 075015, arXiv:1211.2254.

[252] F. Richard, G. Arcadi, and Y. Mambrini, Search for Dark Matter at Colliders, arXiv:1411.0088

[253] T. Daylan, D. P. Finkbeiner, D. Hooper, T. Linden, S. K. N. Portillo, et al., The Characterization of the Gamma-Ray Signal from the Central Milky Way: A Compelling Case for Annihilating Dark Matter, arXiv: 1402.6703

[254] F. Calore, I. Cholis, and C. Weniger, Background model systematics for the Fermi GeV excess, arXiv:1409.0042

[255] S. Biswas, E. Gabrielli, M. Heikinheimo, and B. Mele, Higgs-boson production in association with a Dark Photon in $e^{+} e^{-}$collisions, arXiv:1503.0583

[256] T. Behnke, J. E. Brau, B. Foster, J. Fuster, M. Harrison, et al., The International Linear Collider Technical Design Report - Volume 1: Executive Summary, arXiv:1306.6327

[257] TLEP Design Study Working Group Collaboration, M. Bicer et al., First Look at the Physics Case of TLEP, JHEP 1401 (2014) 164, arXiv:1308.6176.

[258] M. Raggi, V. Kozhuharov, and P. Valente, The PADME experiment at LNF, arXiv:1501.0186

[259] KLOE-2 Collaboration, D. Babusci et al., Search for dark Higgsstrahlung in e+ e- -i mu+ mu- and missing energy events with the KLOE experiment, arXiv:1501.0679

[260] KLOE-2 Collaboration, F. Curciarello, Dark Forces at DA NE, arXiv:1502.0551

[261] Belle Collaboration, I. Jaegle, Search for the dark photon and the dark Higgs boson at Belle, arXiv:1502.0008

[262] R. Essig, J. Mardon, M. Papucci, T. Volansky, and Y.-M. Zhong, Constraining Light Dark Matter with Low-Energy $e^{+} e^{-}$Colliders, JHEP 1311 (2013) 167, arXiv:1309.5084.

[263] N. Chen, J. Wang, and X.-P. Wang, The leptophilic dark matter with $Z^{\prime}$ interaction: from indirect searches to future $e^{+} e^{-}$collider searches, arXiv:1501.0448

[264] I. Hinchliffe, F. Paige, M. Shapiro, J. Soderqvist, and W. Yao, Precision SUSY measurements at CERN LHC, Phys.Rev. D55 (1997) 5520-5540, hep-ph/9610544.

[265] B. Allanach, C. Lester, M. A. Parker, and B. Webber, Measuring sparticle masses in nonuniversal string inspired models at the LHC, JHEP 0009 (2000) 004, hep-ph/0007009.

[266] B. Gjelsten, D. Miller, and P. Osland, Measurement of the gluino mass via cascade decays for SPS 1a, JHEP 0506 (2005) 015, hep-ph/0501033.

[267] H.-C. Cheng, D. Engelhardt, J. F. Gunion, Z. Han, and B. McElrath, Accurate Mass Determinations in Decay Chains with Missing Energy, Phys.Rev.Lett. 100 (2008) 252001, arXiv:0802.4290.

[268] H.-C. Cheng, J. F. Gunion, Z. Han, G. Marandella, and B. McElrath, Mass determination in SUSY-like events with missing energy, JHEP 0712 (2007) 076, arXiv:0707.0030.

[269] K. Kawagoe, M. Nojiri, and G. Polesello, A New SUSY mass reconstruction method at the CERN LHC, Phys. Rev. D71 (2005) 035008, hep-ph/0410160.

[270] C. Lester and D. Summers, Measuring masses of semiinvisibly decaying particles pair produced at hadron colliders, Phys.Lett. B463 (1999) 99-103, hep-ph/9906349.

[271] M. M. Nojiri and M. Takeuchi, Study of the top reconstruction in top-partner events at the LHC, JHEP 0810 (2008) 025, arXiv:0802.4142.

[272] W. S. Cho, K. Choi, Y. G. Kim, and C. B. Park, Gluino Stransverse Mass, Phys.Rev.Lett. 100 (2008) 171801, arXiv:0709.0288.

[273] M. M. Nojiri, Y. Shimizu, S. Okada, and K. Kawagoe, Inclusive transverse mass analysis for squark and gluino mass determination, JHEP 0806 (2008) 035, arXiv:0802.2412.

[274] J. Alwall, A. Freitas, and O. Mattelaer, Measuring Sparticles with the Matrix Element, AIP Conf.Proc. 1200 (2010) 442-445, arXiv:0910.2522.

[275] J. S. Gainer, J. Lykken, K. T. Matchev, S. Mrenna, and M. Park, The Matrix Element Method: Past, Present, and Future, arXiv: 1307.3546

[276] T. Han, I.-W. Kim, and J. Song, Kinematic Cusps: Determining the Missing Particle Mass at Colliders, Phys.Lett. B693 (2010) 575-579, arXiv:0906.5009. 
[277] H. Sun, Dark matter searches in jet plus missing energy events in $\gamma p$ collisions at the CERN LHC, Phys.Rev. D90 (2014) 035018, arXiv:1407.5356.

[278] S. Bilmis, I. Turan, T. Aliev, M. Deniz, L. Singh, et al., Constraints on Dark Photon from Neutrino-Electron Scattering Experiments, arXiv:1502.0776

[279] V. Bednyakov and S. Kovalenko, On possibility for dark matter search with accelerated beam of particles, Unpublished (1999).

[280] T.-F. Feng, X.-Q. Li, W.-G. Ma, J.-X. Wang, and G.-B. Zhao, Detecting the ambient neutralino dark matter particles at accelerator, HEPNP 30 (2006) 12, hep-ph/0610396.

[281] S. Assadi, C. Collins, P. McIntyre, J. Gerity, J. Kellams, et al., Higgs Factory and 100 TeV Hadron Collider: Opportunity for a New World Laboratory within a Decade, arXiv:1402.5973

[282] S. Chattopadhyay, Physics at FAIR, Nucl.Phys. A931 (2014) 267-276.

[283] V. Kekelidze, A. Kovalenko, R. Lednicky, V. Matveev, I. Meshkov, et al., Project NICA at JINR, Nucl.Phys. A904-905 (2013) 945c-948c.

[284] MiniBooNE Collaboration Collaboration, R. Thornton, Accelerator-Produced Dark Matter Search using MiniBooNE, arXiv:1411.4311.

[285] D. E. Soper, M. Spannowsky, C. J. Wallace, and T. M. P. Tait, Scattering of Dark Particles with Light Mediators, Phys.Rev. D90 (2014), no. 11 115005, arXiv:1407.2623.

[286] B. Batell, M. Pospelov, and A. Ritz, Exploring Portals to a Hidden Sector Through Fixed Targets, Phys.Rev. D80 (2009) 095024, arXiv:0906.5614.

[287] R. Essig, R. Harnik, J. Kaplan, and N. Toro, Discovering New Light States at Neutrino Experiments, Phys.Rev. D82 (2010) 113008, arXiv:1008.0636.

[288] B. Batell, M. Pospelov, and A. Ritz, Probing a Secluded U(1) at B-factories, Phys.Rev. D79 (2009) 115008, arXiv:0903.0363.

[289] D. E. Morrissey and A. P. Spray, New Limits on Light Hidden Sectors from Fixed-Target Experiments, JHEP 1406 (2014) 083, arXiv:1402.4817.

[290] Y. Kahn, G. Krnjaic, J. Thaler, and M. Toups, DAEdALUS and Dark Matter, arXiv:1411.1055

[291] LENA Collaboration, M. Wurm et al., The next-generation liquid-scintillator neutrino observatory LENA, Astropart.Phys. 35 (2012) 685-732, arXiv:1104.5620.

[292] J. Berger, Y. Cui, and Y. Zhao, Detecting Boosted Dark Matter from the Sun with Large Volume Neutrino Detectors, arXiv:1410.2246

[293] K. Kong, G. Mohlabeng, and J.-C. Park, Boosted dark matter signals uplifted with self-interaction, Phys.Lett. B743 (2015) 256-266, arXiv:1411.6632.

[294] Super-Kamiokande Collaboration, Y. Fukuda et al., The Super-Kamiokande detector, Nucl.Instrum.Meth. A501 (2003) 418-462

[295] K. Abe, T. Abe, H. Aihara, Y. Fukuda, Y. Hayato, et al., Letter of Intent: The Hyper-Kamiokande Experiment Detector Design and Physics Potential —, arXiv:1109.3262

[296] A. Badertscher, A. Curioni, U. Degunda, L. Epprecht, S. Horikawa, et al., Giant Liquid Argon Observatory for Proton Decay, Neutrino Astrophysics and CP-violation in the Lepton Sector (GLACIER), arXiv:1001.0076

[297] IceCube PINGU Collaboration, M. Aartsen et al., Letter of Intent: The Precision IceCube Next Generation Upgrade (PINGU), arXiv:1401.2046

[298] ANTARES Collaboration, M. Ageron et al., ANTARES: the first undersea neutrino telescope, Nucl.Instrum.Meth. A656 (2011) 11-38, arXiv:1104.1607.

[299] A. Bueno, Z. Dai, Y. Ge, M. Laffranchi, A. Melgarejo, et al., Nucleon decay searches with large liquid argon TPC detectors at shallow depths: Atmospheric neutrinos and cosmogenic backgrounds, JHEP 0704 (2007) 041, hep-ph/0701101.

[300] A. Soffer, Constraints on dark forces from the B factories and low-energy experiments, arXiv:1409.5263

[301] Belle Collaboration, O. Tajima et al., Search for invisible decay of the Upsilon(1S), Phys.Rev.Lett. 98 (2007) 132001, hep-ex/0611041.

[302] BaBar Collaboration, B. Aubert et al., A Search for Invisible Decays of the Upsilon(1S), Phys.Rev.Lett. 103 (2009) 251801, arXiv:0908.2840.

[303] BES Collaboration, M. Ablikim et al., Search for the invisible decay of J / psi in psi(2S) - pi+ pi- J / psi, Phys.Rev.Lett. 100 (2008) 192001, arXiv:0710.0039.

[304] L. Chang, O. Lebedev, and J. Ng, On the invisible decays of the Upsilon and J / Psi resonances, Phys.Lett. B441 (1998) 419-424, hep-ph/9806487.

[305] C. Savage, G. Gelmini, P. Gondolo, and K. Freese, Compatibility of DAMA/LIBRA dark matter detection with other searches, JCAP 0904 (2009) 010, arXiv:0808.3607.

[306] M. Fairbairn and J. Heal, On the complementarity of Dark Matter Searches at Resonance, Phys.Rev. D90 (2014) 115019, arXiv:1406.3288.

[307] M. Wood, J. Buckley, S. Digel, S. Funk, D. Nieto, et al., Prospects for Indirect Detection of Dark Matter with CTA, arXiv: 1305.0302

[308] A. Kounine, The Alpha Magnetic Spectrometer on the International Space Station, Int.J.Mod.Phys. E21 (2012), no. 081230005

[309] M. Gomez, C. Jackson, and G. Shaughnessy, Dark Matter on Top, arXiv:1404.1918

[310] U. Haisch and E. Re, Simplified dark matter top-quark interactions at the LHC, arXiv:1503.0069

[311] B. Dbrich, Looking for dark matter on the light side, arXiv:1501.0327

[312] K. Van Tilburg, N. Leefer, L. Bougas, and D. Budker, Search for ultralight scalar dark matter with atomic spectroscopy, arXiv:1503.0688 
[313] A. Arvanitaki, J. Huang, and K. Van Tilburg, Searching for dilaton dark matter with atomic clocks, Phys.Rev. D91 (2015), no. 1 015015, arXiv:1405.2925.

[314] Y. Stadnik and V. Flambaum, Searching for dark matter and variation of fundamental constants with laser and maser interferometry, Phys.Rev.Lett. 114 (2015), no. 16 161301, arXiv:1412.7801.

[315] M. Khlopov, Dark atoms and puzzles of dark matter searches, Int.J.Mod.Phys. A29 (2014) 1443002.

[316] M. Y. Khlopov and R. Shibaev, Probes for 4th generation constituents of dark atoms in Higgs boson studies at the $L H C, \operatorname{arXiv:1402.0180}$

[317] K. Belotsky, M. Khlopov, and M. Laletin, Dark Atoms and their decaying constituents, arXiv:1411.3657

[318] K. Belotsky, M. Khlopov, C. Kouvaris, and M. Laletin, Decaying Dark Atom constituents and cosmic positron excess, Adv.High Energy Phys. 2014 (2014) 214258, arXiv:1403.1212.

[319] R. Primulando, E. Salvioni, and Y. Tsai, The Dark Penguin Shines Light at Colliders, arXiv:1503.0420.

[320] P. Ko, Dark matter, dark radiation and Higgs phenomenology in the hidden sector DM models, arXiv:1503.0541.

[321] A. Rajaraman, J. Smolinsky, and P. Tanedo, On-Shell Mediators and Top-Charm Dark Matter Models for the Fermi-LAT Galactic Center Excess, arXiv:1503.0591

[322] Q.-F. Xiang, X.-J. Bi, P.-F. Yin, and Z.-H. Yu, Searches for dark matter signals in simplified models at future hadron colliders, arXiv: 1503.0293

[323] V. Martin-Lozano, M. Peiro, and P. Soler, Isospin violating dark matter in Stückelberg portal scenarios, arXiv: 1503.0178

[324] F. Rossi-Torres and C. Moura, Scalar Dark Matter in the light of LEP and ILC Experiments, arXiv:1503.0647

[325] E. Fortes, V. Pleitez, and F. Stecker, Secluded WIMPs, QED with massive photons, and the galactic center gamma-ray excess, arXiv: 1503.0822

[326] J. Suzuki, T. Horie, Y. Inoue, and M. Minowa, Experimental Search for Hidden Photon CDM in the eV mass range with a Dish Antenna, arXiv:1504.0011

[327] A. Delgado, M. Garcia-Pepin, B. Ostdiek, and M. Quiros, Dark Matter from the Supersymmetric Custodial Triplet Model, arXiv: 1504.0248

[328] K. Ghorbani and H. Ghorbani, Two-portal Dark Matter, arXiv:1504.0361.

[329] K. Kainulainen, K. Tuominen, and J. Virkajrvi, A model for dark matter, naturalness and a complete gauge unification, arXiv:1504.0719.

[330] Q.-H. Cao, C.-R. Chen, C. S. Li, and H. Zhang, Effective Dark Matter Model: Relic density, CDMS II, Fermi LAT and LHC, JHEP 1108 (2011) 018, arXiv:0912.4511.

[331] J. Goodman and W. Shepherd, LHC Bounds on UV-Complete Models of Dark Matter, arXiv:1111.2359

[332] I. M. Shoemaker and L. Vecchi, Unitarity and Monojet Bounds on Models for DAMA, CoGeNT, and CRESST-II, Phys.Rev. D86 (2012) 015023, arXiv:1112.5457.

[333] A. Rajaraman, W. Shepherd, T. M. Tait, and A. M. Wijangco, LHC Bounds on Interactions of Dark Matter, Phys.Rev. D84 (2011) 095013, arXiv:1108.1196.

[334] K. Cheung, P.-Y. Tseng, Y.-L. S. Tsai, and T.-C. Yuan, Global Constraints on Effective Dark Matter Interactions: Relic Density, Direct Detection, Indirect Detection, and Collider, JCAP 1205 (2012) 001, arXiv:1201.3402.

[335] R. Cotta, J. Hewett, M. Le, and T. Rizzo, Bounds on Dark Matter Interactions with Electroweak Gauge Bosons, Phys.Rev. D88 (2013) 116009, arXiv:1210.0525.

[336] S. Profumo, W. Shepherd, and T. Tait, Pitfalls of dark matter crossing symmetries, Phys.Rev. D88 (2013), no. 5 056018, arXiv:1307.6277.

[337] A. Alves, S. Profumo, and F. S. Queiroz, The dark $Z^{\prime}$ portal: direct, indirect and collider searches, JHEP 1404 (2014) 063, arXiv:1312.5281.

[338] M. B. Krauss, S. Morisi, W. Porod, and W. Winter, Higher Dimensional Effective Operators for Direct Dark Matter Detection, JHEP 1402 (2014) 056, arXiv:1312.0009.

[339] O. Buchmueller, M. J. Dolan, and C. McCabe, Beyond Effective Field Theory for Dark Matter Searches at the LHC, JHEP 1401 (2014) 025, arXiv:1308.6799.

[340] G. Busoni, A. De Simone, J. Gramling, E. Morgante, and A. Riotto, On the Validity of the Effective Field Theory for Dark Matter Searches at the LHC, Part II: Complete Analysis for the s-channel, JCAP 1406 (2014) 060, arXiv:1402.1275.

[341] G. Busoni, A. De Simone, T. Jacques, E. Morgante, and A. Riotto, On the Validity of the Effective Field Theory for Dark Matter Searches at the LHC Part III: Analysis for the t-channel, JCAP 1409 (2014) 022, arXiv:1405.3101.

[342] F. D'Eramo and M. Procura, Connecting Dark Matter UV Complete Models to Direct Detection Rates via Effective Field Theory, JHEP 1504 (2015) 054, arXiv:1411.3342.

[343] A. Drozd, J. Ellis, J. Quevillon, and T. You, Comparing EFT and Exact One-Loop Analyses of Non-Degenerate Stops, arXiv: 1504.0240

[344] E. Dudas and D. Ghilencea, Effective operators in SUSY, superfield constraints and searches for a UV completion, arXiv: 1503.0831

[345] M. T. Frandsen, F. Kahlhoefer, S. Sarkar, and K. Schmidt-Hoberg, Direct detection of dark matter in models with a light Z', JHEP 1109 (2011) 128, arXiv:1107.2118.

[346] P. Agrawal, S. Blanchet, Z. Chacko, and C. Kilic, Flavored Dark Matter, and Its Implications for Direct Detection and Colliders, Phys.Rev. D86 (2012) 055002, arXiv:1109.3516.

[347] H. An, X. Ji, and L.-T. Wang, Light Dark Matter and Z' Dark Force at Colliders, JHEP 1207 (2012) 182, arXiv: 1202.2894 .

[348] M. T. Frandsen, F. Kahlhoefer, A. Preston, S. Sarkar, and K. Schmidt-Hoberg, LHC and Tevatron Bounds on the Dark Matter Direct Detection Cross-Section for Vector Mediators, JHEP 1207 (2012) 123, arXiv:1204.3839. 
[349] H. An, R. Huo, and L.-T. Wang, Searching for Low Mass Dark Portal at the LHC, Phys.Dark Univ. 2 (2013) 50-57, arXiv:1212.2221.

[350] S. Chang, R. Edezhath, J. Hutchinson, and M. Luty, Effective WIMPs, Phys.Rev. D89 (2014), no. 1 015011, arXiv: 1307.8120 .

[351] H. An, L.-T. Wang, and H. Zhang, Dark matter with t-channel mediator: a simple step beyond contact interaction, Phys.Rev. D89 (2014), no. 11 115014, arXiv:1308.0592.

[352] A. DiFranzo, K. I. Nagao, A. Rajaraman, and T. M. Tait, Simplified Models for Dark Matter Interacting with Quarks, JHEP 1311 (2013) 014, arXiv:1308.2679.

[353] E. Morgante, On the Validity of the EFT for Dark Matter Searches at the LHC, arXiv:1409.6668

[354] S. Malik, C. McCabe, H. Araujo, A. Belyaev, C. Boehm, et al., Interplay and Characterization of Dark Matter Searches at Colliders and in Direct Detection Experiments, arXiv:1409.4075

[355] O. Buchmueller, M. J. Dolan, S. A. Malik, and C. McCabe, Characterising dark matter searches at colliders and direct detection experiments: Vector mediators, arXiv:1407.8257

[356] M. J. Dolan, C. McCabe, F. Kahlhoefer, and K. Schmidt-Hoberg, A taste of dark matter: Flavour constraints on pseudoscalar mediators, arXiv:1412.5174

[357] K. Kong, Measuring Properties of Dark Matter at the LHC, AIP Conf.Proc. 1604 (2014) 381-388, arXiv: 1309.6936.

[358] J. Ellis, The Physics Landscape after the Higgs Discovery at the LHC, arXiv:1504.0365

[359] A. Arbey, M. Battaglia, and F. Mahmoudi, The Higgs boson, Supersymmetry and Dark Matter: Relations and Perspectives, arXiv:1504.0509

[360] Z.-H. Yu, X.-J. Bi, Q.-S. Yan, and P.-F. Yin, Tau Portal Dark Matter models at the LHC, arXiv:1410.3347.

[361] J. Kile, A. Kobach, and A. Soni, Lepton-Flavored Dark Matter, arXiv:1411.1407

[362] E. Ma and A. Natale, Dark Matter with Flavor Symmetry and its Collider Signature, Phys.Lett. B740 (2014) 80-82, arXiv: 1410.2902 .

[363] J. Bramante, P. J. Fox, A. Martin, B. Ostdiek, T. Plehn, et al., The Relic Neutralino Surface at a 100 TeV collider, arXiv:1412.4789

[364] L. Calibbi, J. M. Lindert, T. Ota, and Y. Takanishi, LHC Tests of Light Neutralino Dark Matter without Light Sfermions, JHEP 1411 (2014) 106, arXiv:1410.5730

[365] F. S. Queiroz, K. Sinha, and A. Strumia, Leptoquarks, Dark Matter, and Anomalous LHC Events, arXiv:1409.6301

[366] G. Busoni, A. De Simone, T. Jacques, E. Morgante, and A. Riotto, Making the Most of the Relic Density for Dark Matter Searches at the LHC 14 TeV Run, arXiv:1410.7409

[367] M. R. Buckley, D. Feld, and D. Goncalves, Scalar Simplified Models for Dark Matter, Phys.Rev. D91 (2015), no. 1 015017, arXiv:1410.6497.

[368] P. Harris, V. V. Khoze, M. Spannowsky, and C. Williams, Constraining Dark Sectors at Colliders: Beyond the Effective Theory Approach, arXiv:1411.0535

[369] Y.-B. Liu and Z.-J. Xiao, Constraining dark matter in the LRTH model with latest LHC, XENON100 and LUX date, arXiv: 1409.8000

[370] M. Low and L.-T. Wang, Neutralino dark matter at $14 \mathrm{TeV}$ and $100 \mathrm{TeV}$, JHEP 1408 (2014) 161, arXiv: 1404.0682.

[371] J. Abdallah, A. Ashkenazi, A. Boveia, G. Busoni, A. De Simone, et al., Simplified Models for Dark Matter and Missing Energy Searches at the LHC, arXiv:1409.2893

[372] T. Han, Z. Liu, and S. Su, Light Neutralino Dark Matter: Direct/Indirect Detection and Collider Searches, JHEP 1408 (2014) 093, arXiv:1406.1181.

[373] A. Bhattacharya, R. Gandhi, and A. Gupta, The Direct Detection of Boosted Dark Matter at High Energies and $\mathrm{PeV}$ events at IceCube, Unpublished, (2014) arXiv:1407.3280.

[374] J. Blumenthal, P. Gretskov, M. Krmer, and C. Wiebusch, Effective field theory interpretation of searches for dark matter annihilation in the Sun with the IceCube Neutrino Observatory, arXiv:1411.5917

[375] T. Li, S. Miao, and Y.-F. Zhou, Light mediators in dark matter direct detections, arXiv:1412.6220

[376] K. Agashe, Y. Cui, L. Necib, and J. Thaler, (In)direct Detection of Boosted Dark Matter, JCAP 1410 (2014), no. 10 062, arXiv: 1405.7370

[377] N. F. Bell, Y. Cai, J. B. Dent, R. K. Leane, and T. J. Weiler, Dark matter at the LHC: EFTs and gauge invariance, arXiv:1503.0787

[378] A. Ibarra and S. Wild, Dirac dark matter with a charged mediator: a comprehensive one-loop analysis of the direct detection phenomenology, arXiv: 1503.0338

[379] M. Garny, A. Ibarra, and S. Vogl, Signatures of Majorana dark matter with t-channel mediators, arXiv:1503.0150

[380] A. Crivellin, U. Haisch, and A. Hibbs, LHC constraints on gauge boson couplings to dark matter, arXiv:1501.0090

[381] A. J. Anderson, P. J. Fox, Y. Kahn, and M. McCullough, Halo-Independent Direct Detection Analyses Without Mass Assumptions, arXiv:1504.0333. 